\title{
Performance of Real-Time Remote Control Systems in the ISM Band
}

by

\author{
Abdallah Ismail, B.Eng. \\ A Thesis submitted to the \\ Faculty of Graduate Studies and Research \\ in partial fulfillment of the requirements for the degree of \\ Master of Applied Science in Electrical Engineering
Ottawa-Carleton Institute for Electrical and Computer Engineering
Department of Systems and Computer Engineering
Carleton University
Ottawa, Ontario, Canada

January 2009

(C)Abdallah Ismail, 2009 
Library and

Archives Canada

Published Heritage

Branch

395 Wellington Street

Ottawa ON K1A 0N4

Canada
Bibliothèque et

Archives Canada

Direction du

Patrimoine de l'édition

395 , rue Wellington

Ottawa ON K1A ON4

Canada

Your file Votre référence

ISBN: 978-0-494-52037-6

Our file Notre référence

ISBN: 978-0-494-52037-6

NOTICE:

The author has granted a nonexclusive license allowing Library and Archives Canada to reproduce, publish, archive, preserve, conserve, communicate to the public by telecommunication or on the Internet, loan, distribute and sell theses worldwide, for commercial or noncommercial purposes, in microform, paper, electronic and/or any other formats.

The author retains copyright ownership and moral rights in this thesis. Neither the thesis nor substantial extracts from it may be printed or otherwise reproduced without the author's permission.
AVIS:

L'auteur a accordé une licence non exclusive permettant à la Bibliothèque et Archives Canada de reproduire, publier, archiver, sauvegarder, conserver, transmettre au public par télécommunication ou par l'Internet, prêter, distribuer et vendre des thèses partout dans le monde, à des fins commerciales ou autres, sur support microforme, papier, électronique et/ou autres formats.

L'auteur conserve la propriété du droit d'auteur et des droits moraux qui protège cette thèse. $\mathrm{Ni}$ la thèse ni des extraits substantiels de celle-ci ne doivent être imprimés ou autrement reproduits sans son autorisation.
In compliance with the Canadian

Privacy Act some supporting forms may have been removed from this thesis.

While these forms may be included in the document page count, their removal does not represent any loss of content from the thesis.
Conformément à la loi canadienne sur la protection de la vie privée, quelques formulaires secondaires ont été enlevés de cette thèse.

Bien que ces formulaires aient inclus dans la pagination, il n'y aura aucun contenu manquant.

\section{Canada'}




\section{Abstract}

In remote control applications, a user controls a number of inputs which wirelessly trigger a real-time proportional movement in the controlled object. Such applications are generally referred to as Radio Control (RC) applications. Imperfections in the wireless channel (noise and interference) create a random delay (latency) in the response of the radio control system. A lag occurs when this system latency exceeds a real-time threshold. The System Lag Occurrence Probability (SLOP) is the probability of lag occurrence used to characterize performance of real-time radio control applications. This thesis derives SLOP for a frequency hopping radio control system.

Advanced RC applications use the license-free ISM band for wireless access. Single-chip Commercial-Off-The-Shelf wireless transceivers are used in practice to implement radio control systems operating in the ISM band. ISM transceivers can be classified into five generations depending on the amount of silicon integration available in the transceiver. ISM transceivers offer a variety of techniques to battle noise and interference in the ISM band. Due to hardware limitations found in ISM transceivers, frequency hopping is found to be the only available interference-resistance technique which enables the maximization of interference-resistance in the band.

There are two modes of operation in a frequency hopping system: acquisition and tracking. This thesis studies the effect of using uniform serial acquisition and $\mathrm{N}$-state lock detector tracking on the system latency of a radio control system. 


\section{Acknowledgements}

I am eternally grateful to my parents' never-ending encouragement, support, and love. Without them, this work would not have been possible.

I am grateful to my thesis supervisor, Professor Ioannis Lambadaris, and co-supervisor, Professor Chung-Horng Lung. Their guidance and useful insight contributed significantly to the success of this research. Their in-depth knowledge around radio control systems proved very helpful during this work.

I would like to thank Texas Instruments, Cypress Semiconductor, C-Link Systems, LS Research, and Analog Devices for donating various wireless development kits to this research. Their contributions led to the detailed experiments carried out in this thesis.

Finally, I gratefully acknowledge the technical and financial support received from Dr. Nisith Goel, Mr. Pulak Dhar, and Mr. Brian Stacey at Cistel Technology Inc., as well as MITACS and the Government of Ontario towards the completion of this degree. 


\section{Contents}

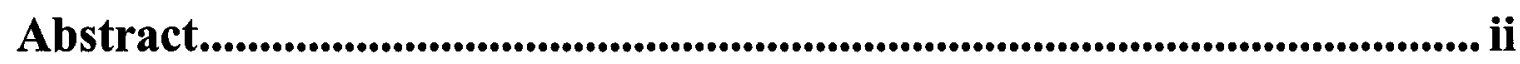

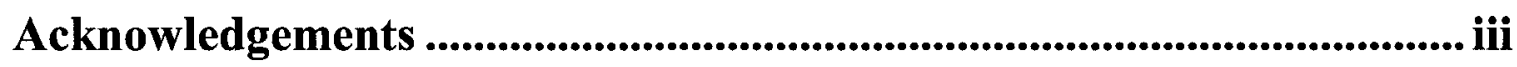

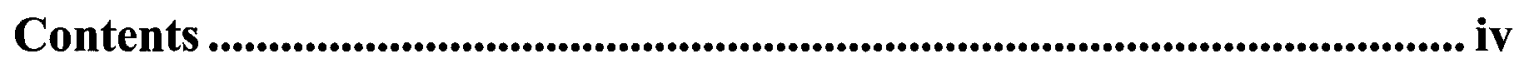

List of Figures...................................................................................... vi

List of Tables .............................................................................................. viii

List of Abbreviations ........................................................................................ ix

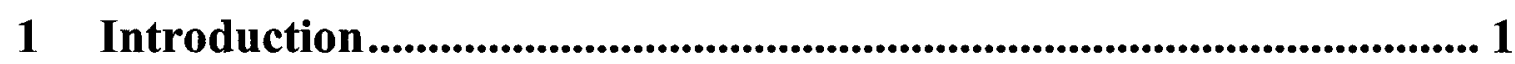

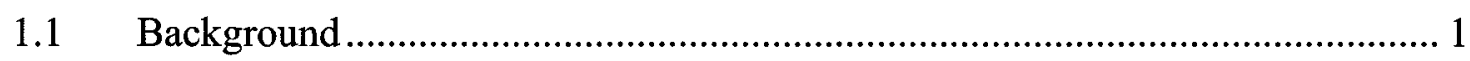

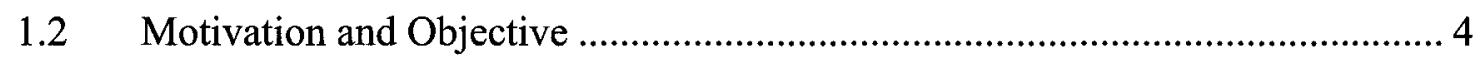

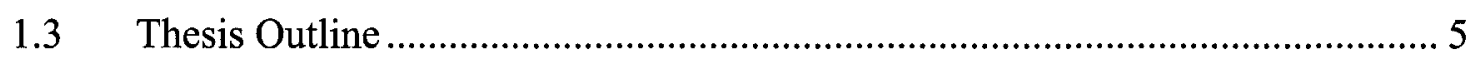

2 State-of-the-Art in ISM Transceiver Technology .............................. 7

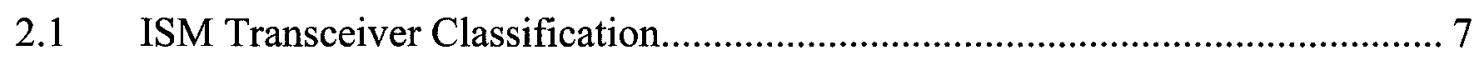

2.2 Available Interference-Resistance Techniques............................................... 17

3 Performance Metric for System Latency ........................................... 22

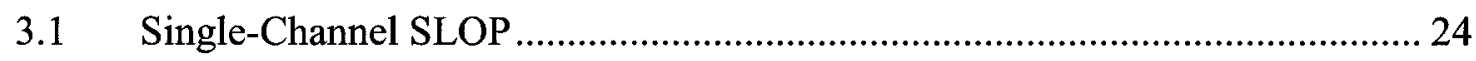

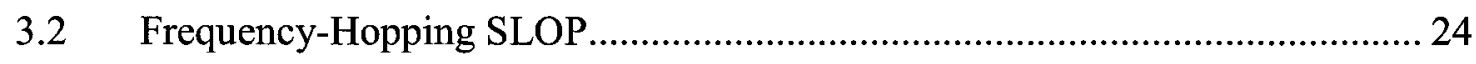

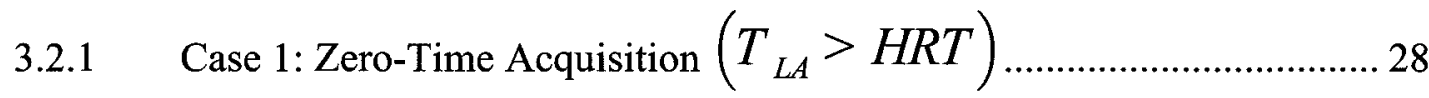


3.2.2 Case 2: Non-Zero-Time Acquisition $\left(T_{L A} \leq H R T\right)$ 29

4 Simulation Setup and Results ................................................................. 38

4.1 Packet-Based Frequency Hopping Simulator ……………………………….... 38

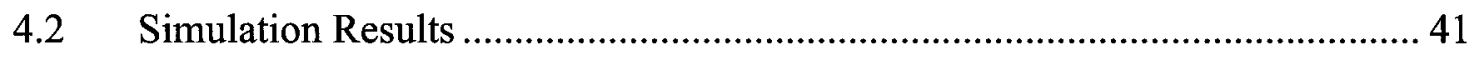

5 Implementation ...................................................................... 49

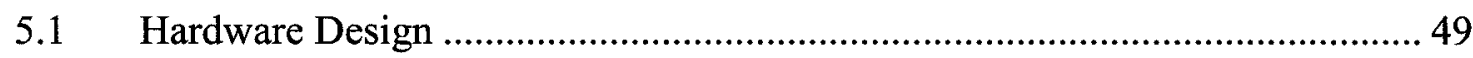

5.1.1 Programmable System-on-Chip.......................................................... 50

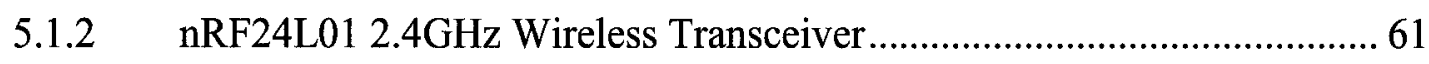

5.1.3 Development Boards used in Prototyping ................................................. 67

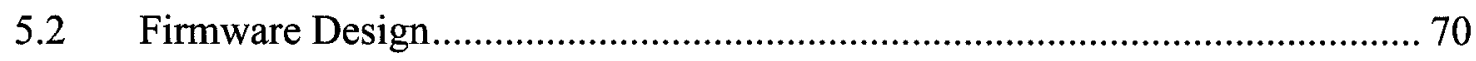

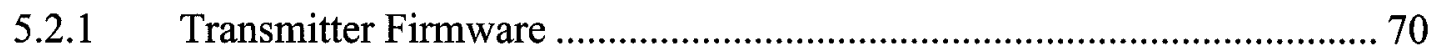

5.2.2 Receiver Firmware .......................................................................... 72

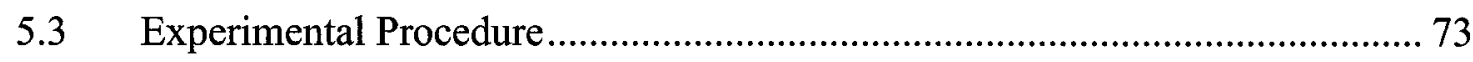

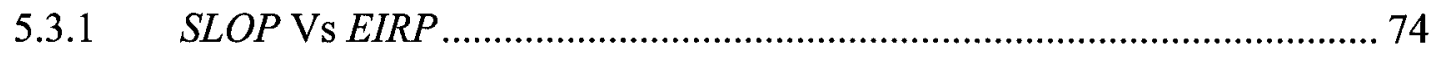

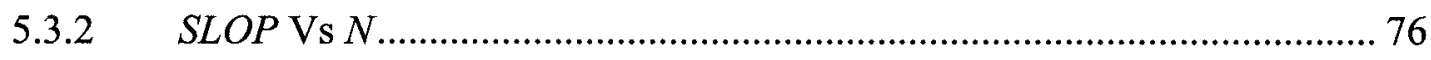

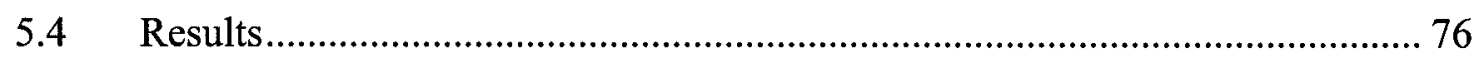

6 Summarized Conclusion and Future Work ....................................... 79 


\section{List of Figures}

Figure 1: An example of a Radio Control application ............................................... 1

Figure 2: A simplified block diagram of the ISM transceiver .................................... 8

Figure 3: Minimum fields required for packet-based wireless communication ................ 8

Figure 4: ISM transceiver architecture classified into five generations ........................ 10

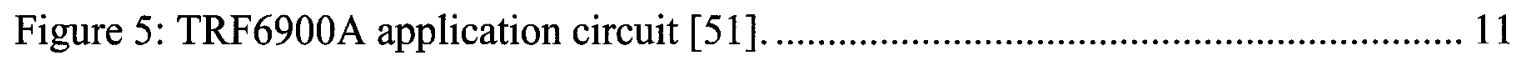

Figure 6: CYWUSB6935 is an example of a second-generation transceiver [17].......... 13

Figure 7: The CC1100 from TI is an example of a third-generation transceiver [9]....... 14

Figure 8: the nRF24E1 is an example of a fourth-generation transceiver [37]............. 15

Figure 9: the nRF24LU1 SoC is an example of fifth-generation transceivers [39]........ 16

Figure 10: Typical interface between transceiver and power amplifier [35].................. 19

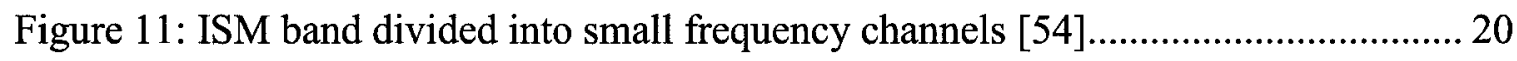

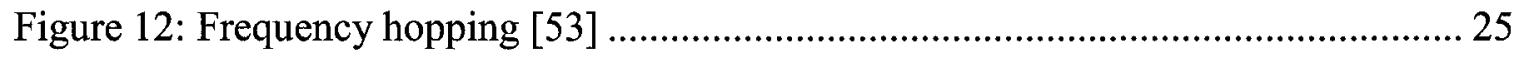

Figure 13: Average lock-in and acquisition times of a frequency hopping system......... 26

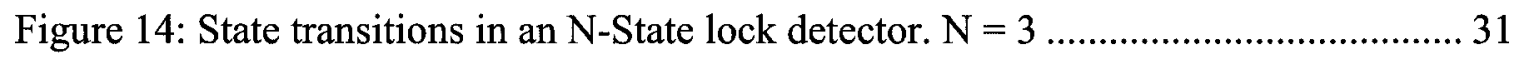

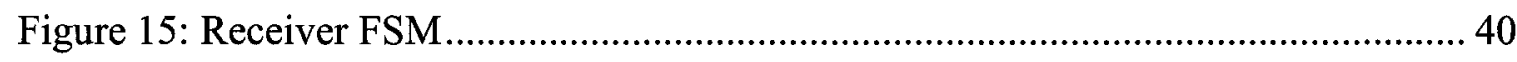

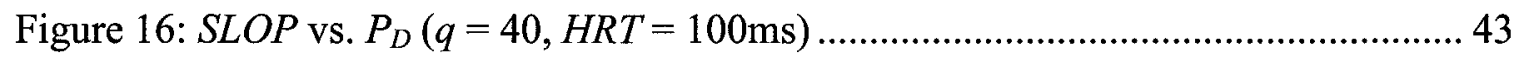

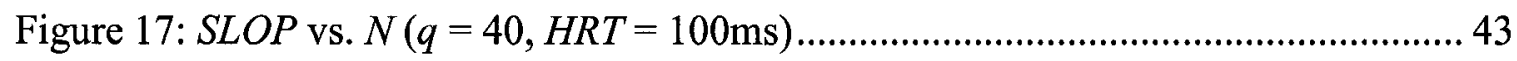

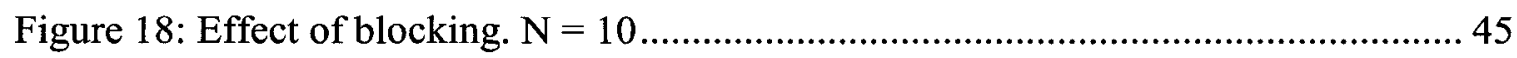

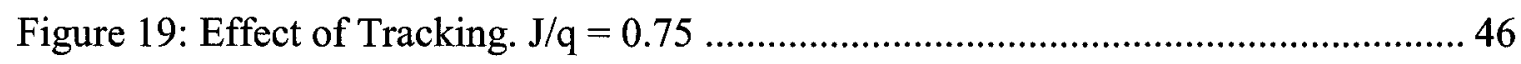

Figure 20: Interference resistance due to increasing number of channels, $\mathrm{N}=10 \ldots \ldots \ldots .47$

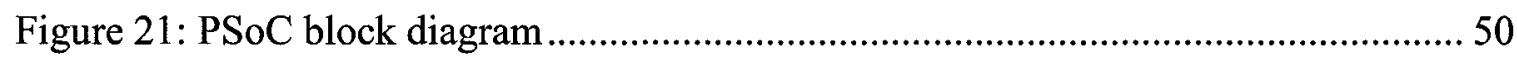




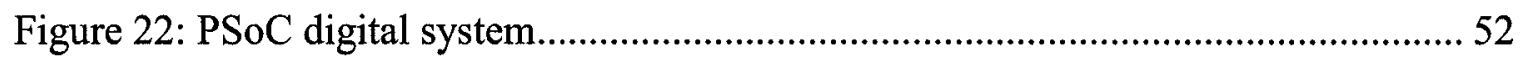

Figure 23: DB00 is configured as a PWM whose output is routed to pin 0 of port $0 \ldots . .53$

Figure 24: Digital PSoC Block Row Structure .............................................................. 54

Figure 25: DB00 is configured as an input-capture timer whose input is fed from P0.0. 55

Figure 26: Example of LUT0 configuration ................................................................ 55

Figure 27: Digital Block Top Level Block Diagram ................................................. 57

Figure 28: Selected (Yet-to-be-placed) user modules ....................................................... 60

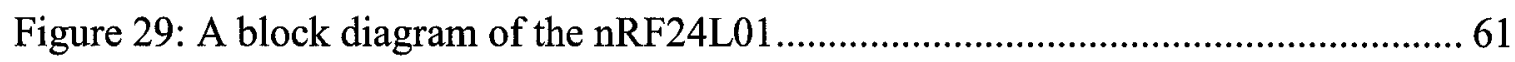

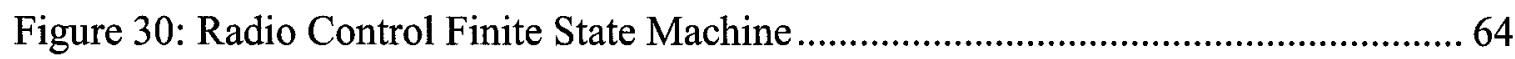

Figure 31: Packet format supported by the nRF24L01 …..............................................6 65

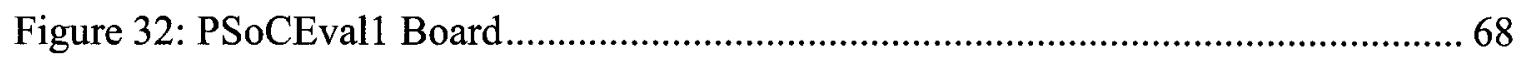

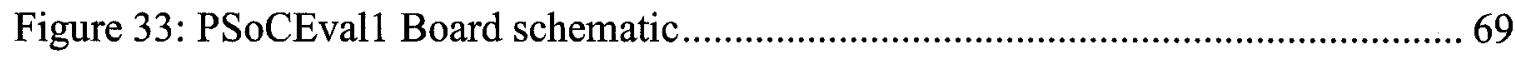

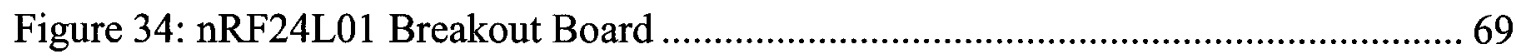

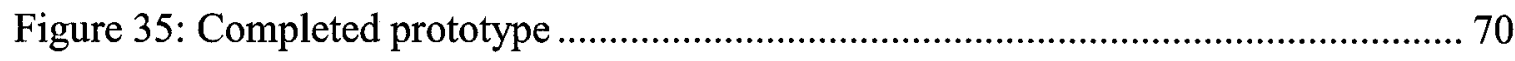

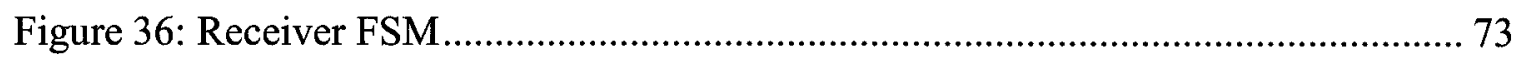

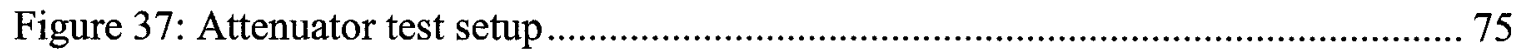

Figure 38: Test setup for measuring Received Power ..................................................... 76

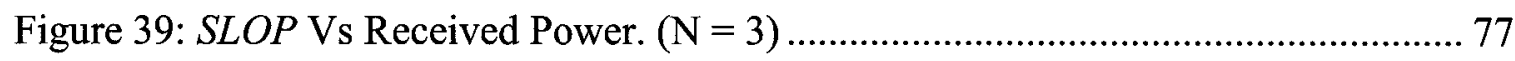

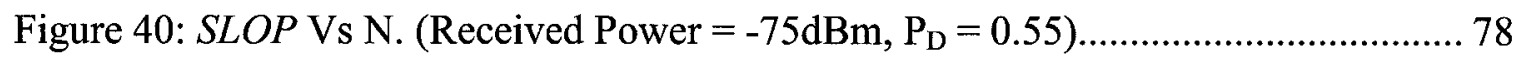




\section{List of Tables}

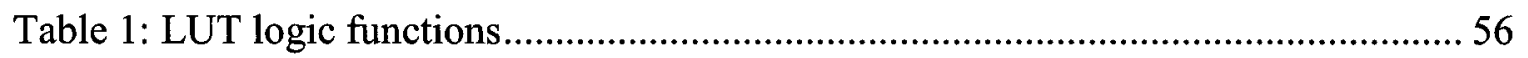

Table 2: Control Register Description ......................................................................... 58

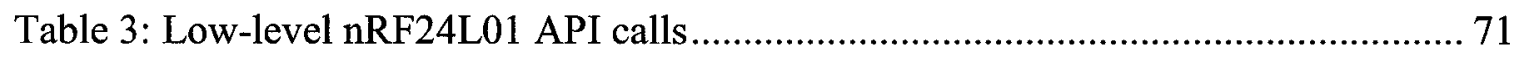

Table 4: High-level nRF24L01 API calls .................................................................... 71 


\section{List of Abbreviations}

$\begin{array}{ll}\text { ACK } & \text { Acknowledgement } \\ \text { ACQ } & \text { Acquisition } \\ \text { ADC } & \text { Analog to Digital Converter } \\ \text { AES } & \text { Advanced Encryption Engine } \\ \text { API } & \text { Application Programming Interface } \\ \text { ARQ } & \text { Automatic Repeat Request } \\ \text { AWGN } & \text { Additive White Gaussian Noise } \\ \text { BER } & \text { Bit Error Rate } \\ \text { BOM } & \text { Bill of Material } \\ \text { CDMA } & \text { Code Division Multiple-Access } \\ \text { CE } & \text { Chip Enable } \\ \text { COTS } & \text { Commercial-Off-The-Shelf } \\ \text { DPU } & \text { Central Processing Unit } \\ \text { DRC } & \text { Cyclic Redundancy Check } \\ \text { CSN } & \text { Chip Select } \\ \text { DAC } & \text { Digital to Analog Converter } \\ \text { DB } & \text { Digital Block } \\ \text { Direct Current }\end{array}$




$\begin{array}{ll}\text { ETSI } & \text { European Telecommunications Standards International } \\ \text { FCC } & \text { Federal Communications Commission } \\ \text { FDMA } & \text { Frequency Division Multiple-Access } \\ \text { FEC } & \text { Forward Error Correction } \\ \text { FHSS } & \text { Frequency Hopping Spread Spectrum } \\ \text { FIFO } & \text { First In First Out } \\ \text { FSM } & \text { Finite State Machine } \\ \text { GDI } & \text { Global Digital Interconnect } \\ \text { GFSK } & \text { Gaussian Frequency Shift Keying } \\ \text { ISR } & \text { Interrupt Service Routine } \\ \text { ISI } & \text { Global In Even } \\ \text { GIO } & \text { Global In Odd } \\ \text { IDO } & \text { Integrated Circuit } \\ \text { HO } & \text { Global Out Even } \\ \text { GOO } & \text { Global Out Odd } \\ \text { HEX } & \text { Graphical User Interface } \\ \text { Hexadecimal file format }\end{array}$




\begin{tabular}{|c|c|}
\hline LCD & Liquid Crystal Display \\
\hline LED & Light Emitting Diode \\
\hline LUT & Look Up Table \\
\hline MAC & Multiply-Accumulate \\
\hline $\mathrm{MCU}$ & Microcontroller Unit \\
\hline MISO & Master In Slave Out \\
\hline MOSI & Master Out Slave In \\
\hline PA & Power Amplifier \\
\hline PD & Power Down \\
\hline$P_{D}$ & Probability of detection \\
\hline PER & Packet Error Rate \\
\hline PID & Packet Identification \\
\hline PLL & Phase Lock Loop \\
\hline PPM & Pulse-Position Modulation \\
\hline PRS & Pseudo Random Sequence \\
\hline PRX & Primary Receiver \\
\hline PSoC & Programmable System on Chip \\
\hline PTX & Primary Transmitter \\
\hline PWM & Pulse Width Modulation \\
\hline $\mathrm{QFN}$ & Quad Flat No-lead package \\
\hline RAM & Random Access Memory \\
\hline $\mathrm{RC}$ & Radio Control \\
\hline RDI & Row Digital Interconnect \\
\hline
\end{tabular}




\begin{tabular}{ll} 
RF & Radio Frequency \\
RI & Row Input \\
ROM & Read Only Memory \\
RX & Receiver \\
SCK & Synchronous Clock \\
SLOP & System Lag Occurrence Probability \\
SMP & Switch Mode Pump \\
SNR & Signal to Noise Ratio \\
SoC & System on Chip \\
SPI & Serial Peripheral Interface \\
STDBY & Standby \\
TX & Transmitter \\
UART & Universal Asynchronous Receive Transmit \\
USB & Universal Serial Bus \\
VLSI & Voltage Controlled Oscillator \\
& Very Large Silicon Integration \\
\hline
\end{tabular}




\section{Introduction}

\subsection{Background}

In remote control applications, a user controls a number of inputs which trigger a proportional movement in servomechanisms mounted in the controlled device. Communication between the user the controlled device is established over a wireless link. The servomechanisms in the controlled device move the control surfaces of the device thus causing it to maneuver in its medium. Such applications are generally referred to as real-time Radio Control ( $\mathrm{RC}$ ) applications. Examples of real-time $\mathrm{RC}$ applications include model car Radio Control and Unmanned Aerial Vehicles (UAV) [29].

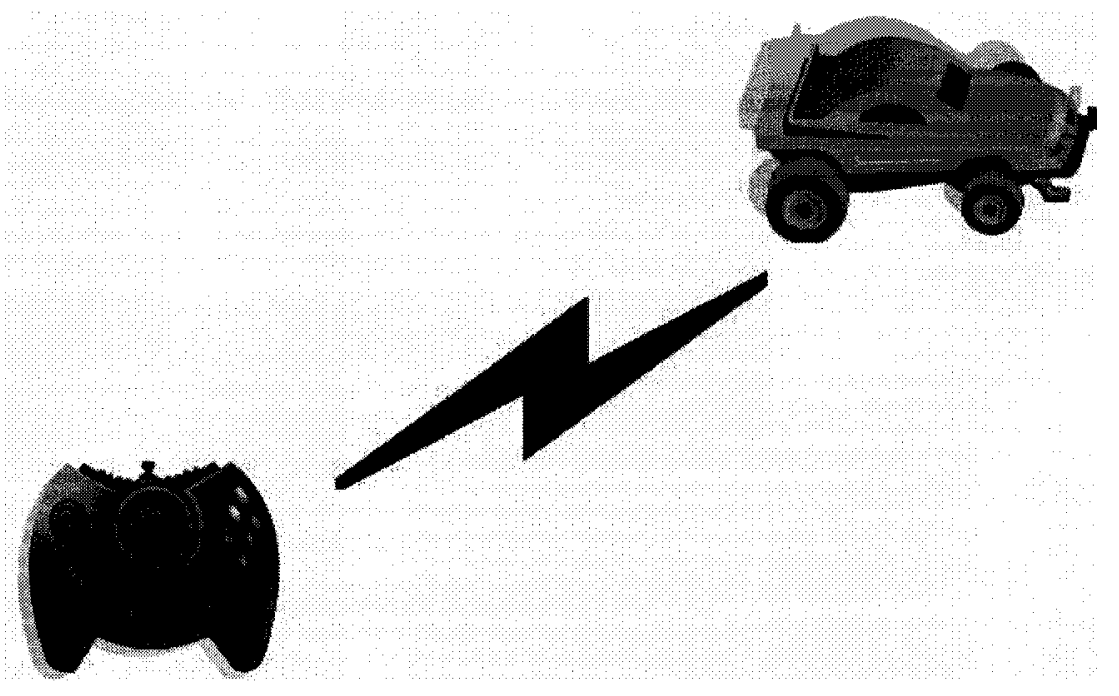

Figure 1: An example of a Radio Control application

Real-time radio control requires that the system respond to user inputs with minimum latency. The time it takes the system to respond to a user input is here referred to as the system latency. System latency can be defined as the inter-arrival time of control 
information from the transmitter to the receiver. Imperfections in the wireless channel (for example noise and interference) results in random latency in the response of the system. Therefore, the robustness of the wireless link established between the transmitter and the receiver has a major effect on the system latency.

A lag is here defined as an event which occurs when the system latency exceeds a specified real-time threshold. In applications where input is being applied by a user (human), the real-time threshold becomes the Human Response Time (HRT) [25]. $H R T$ is a time threshold above which the user notices a lag, but below which the user perceives response as instantaneous. HRT shows up in other real-time applications that interface with users such as Voice-over-IP (VoIP). It is generally estimated to be around $100 \mathrm{~ms}$. The user experience can be defined in terms of the probability of lag occurrence during a radio control session. This thesis derives a performance metric for real-time radio control applications based on the probability of lag occurrence.

Traditional radio control uses analog Pulse-Position Modulated (PPM) signal transmission over reserved frequency bands such as the $72 \mathrm{MHz}$ band in North America and the $35 \mathrm{MHz}$ band in Europe [5].

In recent years, there has been a shift in the RC industry towards the use of digital spread spectrum technology in the $2.4 \mathrm{GHz}$ Industrial, Scientific, and Medical (ISM) band. The $2.4 \mathrm{GHz}$ ISM band is a license-free range of frequencies regulated by the Federal Communications Commission (FCC) in North America [24]. Operation in the ISM band 
requires that the system tolerates interference [47]. With Spread Spectrum, immunity to interference is achieved inherently by the system's spreading gain [7]. The relatively large available bandwidth in the $2.4 \mathrm{GHz}$ ISM band has made it a very attractive wireless medium for both hobbyists and manufacturers alike.

In North America, the FCC is the body responsible for regulating the $2.4 \mathrm{GHz}$ band to limit the potential for interference to licensed operations by low-power non-licensed transmitters [24]. Operation in this band is documented in FCC Part 15 and is subject to two conditions [47]. Firstly, the device may not cause harmful interference. Secondly the device must accept any interference received, including interference that may cause undesired operation.

Frequency Hopping Spread Spectrum (FHSS) technology fulfils both conditions mentioned above [43]. Firstly, frequency hopping limits harmful interference by spreading its signal power across a wider bandwidth. Secondly, frequency hopping tolerates interference by switching communication to free channels. Moreover, frequency hopping offers resiliency to frequency-selective fading (multipath) [46]. It also enables multiple users to access the band through Code-Division Multiple Access (CDMA) operation [43].

Frequency hopping is a relatively simple technology that can be readily implemented using low-cost off-the-shelf components [22]. The aforementioned advantages of frequency hopping make it a feasible and attractive solution for use in real-time radio 
control applications. The FCC defines frequency hopping operation in Part 15.247 $[47][24]$. This thesis studies the effect of using frequency hopping on the probability of lag occurrence in real-time radio control applications.

In practice, Commercial-Off-The-Shelf (COTS) wireless transceivers are used to establish a communication link between the transmitter-receiver pair of a radio control application. A large variety of such transceivers exist today in the market from many different vendors, each offering a unique mix of features and capabilities. Such transceivers are here referred to as ISM Transceivers. This thesis surveys the state of the art in ISM transceiver technology, studies the effect of using such transceivers in the design of real-time radio control systems, and presents a frequency hopping implementation of a real-time radio control system operating in the $2.4 \mathrm{GHz}$ ISM band.

\subsection{Motivation and Objective}

The main motivation of this thesis is to enable the design of practical and efficient realtime radio control systems. Practicality here implies the use of ISM transceivers in the design of such systems. As will be shown in Chapter 2, the use of ISM transceivers necessitates the use of frequency hopping in the design of radio control systems operating in the ISM band.

Efficiency here implies the use of a performance metric to characterize different designs and enable the comparison between them in terms of their performance. Chapter 3 defines the performance metric as the probability of lag occurrence during a radio control session. This thesis deals mainly with studying the effect of frequency hopping on the 
probability of lag occurrence in real-time radio control systems operating in the ISM band. Much of the work done in this thesis is novel, due to the specialization of the field. The thesis aims at establishing the ground work for future work to come in this field. Specifically, the thesis aims to accomplish the following:

- Survey state of the art in ISM transceiver technology, and present available techniques for interference-resistance.

- Derive a performance metric based on the probability of lag occurrence during a frequency hopping real-time radio control session.

- Validate the derivations through simulation and implementation experiments, and study the effect of various design variables on the performance of the system.

- Present an implementation of a packet-based frequency hopping real-time radio control system operating in the $2.4 \mathrm{GHz}$ ISM band.

\subsection{Thesis Outline}

Rest of the thesis is organized as follows:

Chapter 2: Presents a comprehensive survey on the state of the art in ISM transceivers. It classifies the system-level architecture of ISM transceivers into five generations, depending mainly on how much silicon integration is available in the transceiver. This chapter also looks at available interference-resistance techniques, and studies some of the implications of using such transceivers in real-time radio control applications.

Chapter 3: Derives a performance metric for real-time radio control systems based on the probability of lag occurrence. The main part of this chapter deals with frequency hopping systems. 
Chapter 4: Presents simulation results to validate the mathematical derivations made in Chapter 3 and study the effect of various design variables on the performance of the system.

Chapter 5: Presents an implementation of a radio control system operating in the 2.4GHz ISM band. This chapter also presents experimental results which validate the mathematical derivations made in Chapter 3 and the simulation results presented in Chapter 4. 


\section{State-of-the-Art in ISM Transceiver Technology}

One of the goals of this thesis is to provide a practical approach for designing an efficient real-time radio control system. The ISM transceiver is a major building block in the design of radio control systems operating in the ISM band. The ISM transceiver is a lowcost, Commercial-Off-The-Shelf (COTS), single-chip wireless transceiver used to establish packet-based communication in the ISM band. This chapter surveys the state of the art in ISM transceivers and studies the effect of using such transceivers in radio control system design.

\subsection{ISM Transceiver Classification}

From a system-level viewpoint, a packet-based wireless communication system can be shown to consist of three main components: a packet-handler, a serdes (serializer/deserializer), and an RF modem. A simplified block diagram of such a system is shown below in Figure 2. As will be shown later, ISM transceivers originally contained only the RF modem component. In time, ISM transceivers evolved to integrate the packet handler and the serdes. Below is a brief description of each of those components. 


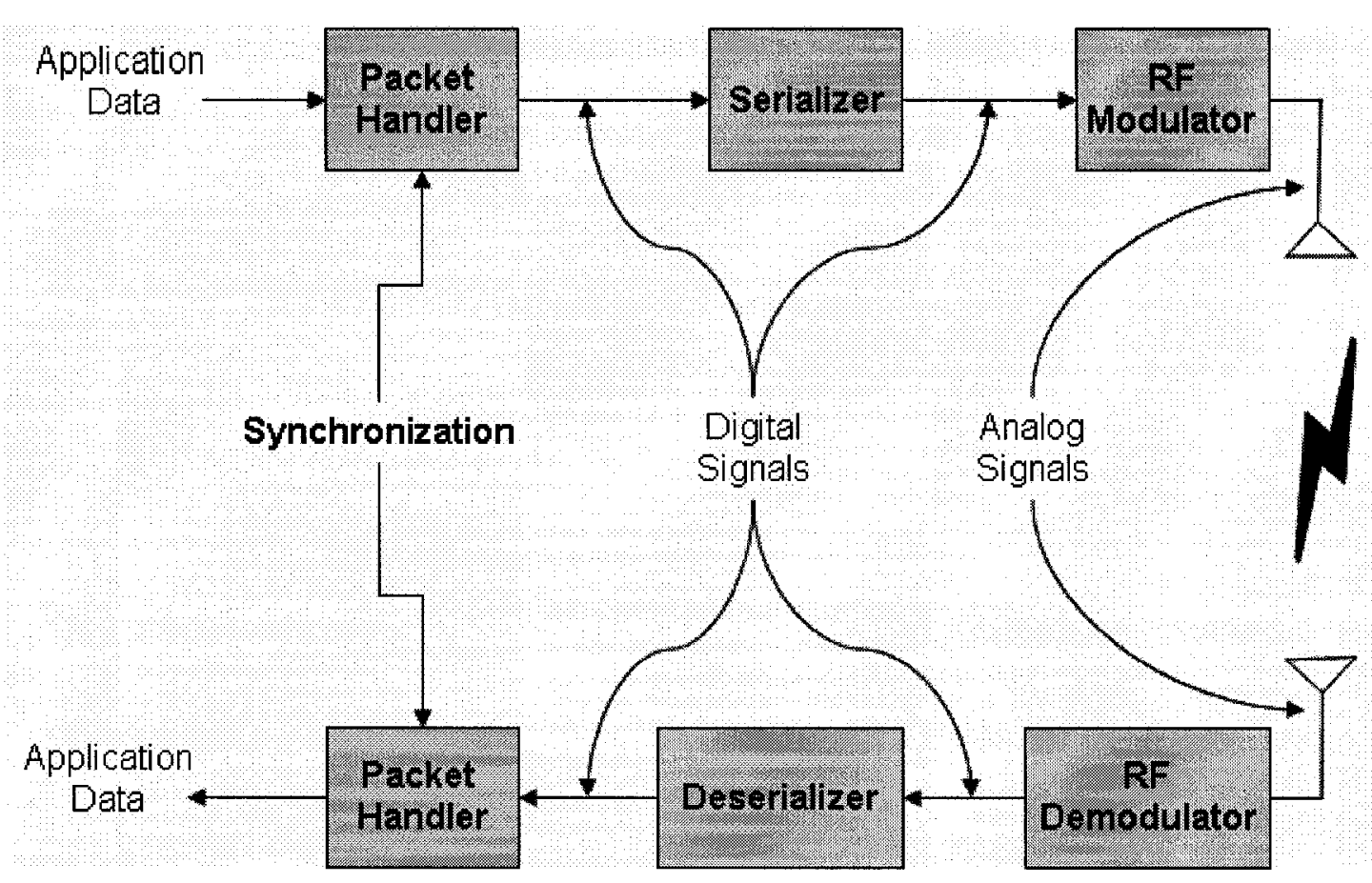

Figure 2: A simplified block diagram of the ISM transceiver

Packet Handler The packet handler controls packet formation and extraction. Packets used in wireless communication contain a minimum number of fields, each of which has a unique purpose. These fields are shown in Figure 3 below. The preamble is a short sequence of alternating ones and zeros placed at the front of the packet in the transmitter to aid the receiver in bit-synchronization. The sync is a predefined sequence placed after the preamble in the transmitter to aid the receiver in byte-synchronization. The payload contains application data. The CRC field is used for error detection and correction.

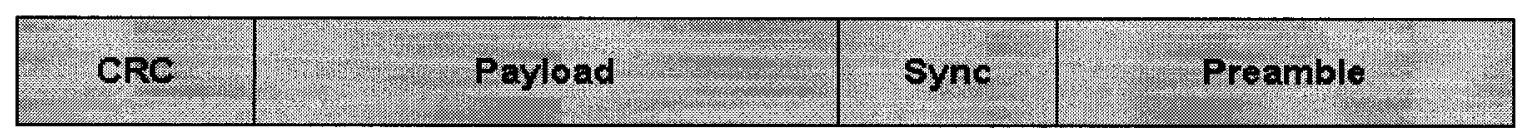

Figure 3: Minimum fields required for packet-based wireless communication 
In transmit mode, the packet-handler wraps the preamble, the sync, and the CRC fields around the application data, thus forming a packet. In receive mode, the packet-handler uses the preamble to achieve bit-synchronization. It then searches for the sync field to achieve byte-synchronization. After bit/byte synchronization is acquired, the packet handler reads the rest of the packet. It then uses the CRC field to check whether the packet is correct or corrupt. If correct, the packet-handler extracts the application data from the payload field and forwards it to the application.

Serdes Packets are stored in memory buffers. The serdes converts packets into a serial bit stream and vice versa to/from memory buffers. In transmit mode, the serdes serializes the packet formed in the packet-handler into a serial bit-stream. The bit period generated by the serdes block controls the overall data-rate. In receive mode, the serdes block passes the serial bit stream to the packet handler.

RF Modem In transmit mode, the RF modem modulates the serial bit stream into an RF signal suitable for wireless transmission in the ISM band. In receive mode, the RF modem de-modulates/detects the wireless signal into a serial bit stream. Note that the RF modem is the only analog part of the system. The rest of the system is digital.

In practice, an ISM transceiver is used to implement the packet handler, serdes, and RF modem components of a packet-based wireless communication system. As mentioned previously, the ISM transceiver is a low-cost, Commercial-Off-The-Shelf (COTS), single-chip wireless transceiver used to establish packet-based communication in the ISM 
band. Rapid growth experienced in the wireless communication industry led to a spur in the development of ISM transceivers. Originally, ISM transceivers consisted only of the RF modem. An external baseband processor was needed to perform packet handling and serdes tasks. As the ISM band grew more and more in popularity, ISM transceivers evolved to integrate packet handling and serdes capabilities, in addition to a rich set of application-specific features, as will be shown later in this chapter.

Currently available ISM transceivers can be classified into five generations, depending on the amount of silicon integration available in the transceiver. A block diagram showing the five transceiver generations as a continuum of increasing silicon integration is shown in the figure below. What follows is a description of the five ISM transceiver generations.

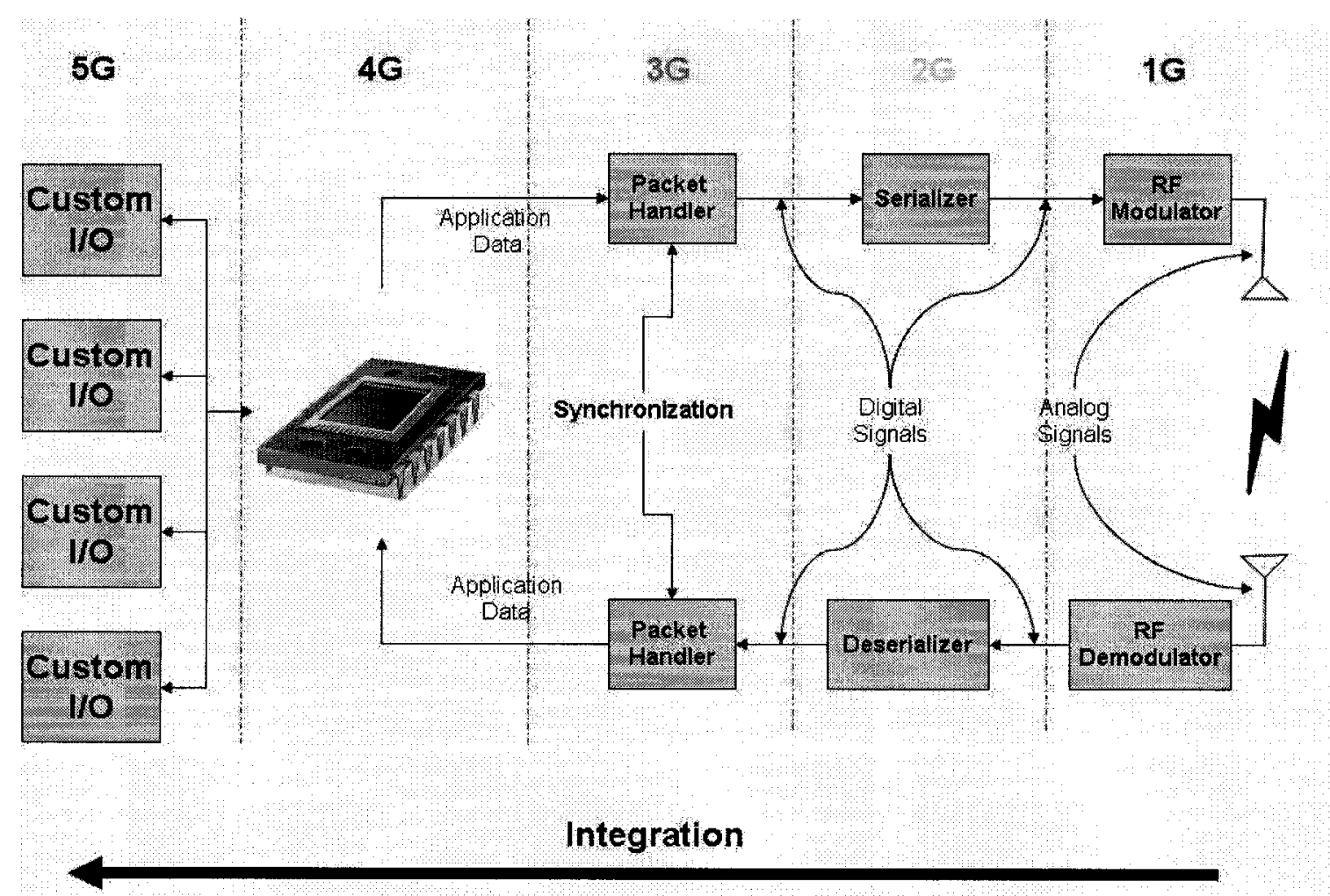

Figure 4: ISM transceiver architecture classified into five generations 
First-generation transceivers consist mainly of the RF modem. The TRF6900 from Texas Instruments [51], the ADF7025 from Analog Devices [2], and the ATR2604 from Atmel [6] are examples of first-generation transceivers.

The on-chip digital capabilities of first generation transceivers are minimal. Serialization/de-serialization, packet-handling, and bit-byte synchronization must be handled using an external baseband processor [41].

Figure 5 below shows an application circuit of the TRF6900A [51]. A general trend found in first generation transceivers is to leave much of the transceiver's supporting circuitry to be added externally by the user. This requires a considerable amount of analog and RF design expertise of course. Second generation transceivers solve this issue by integrating most of the supporting circuitry in the transceiver, as will be shown shortly.

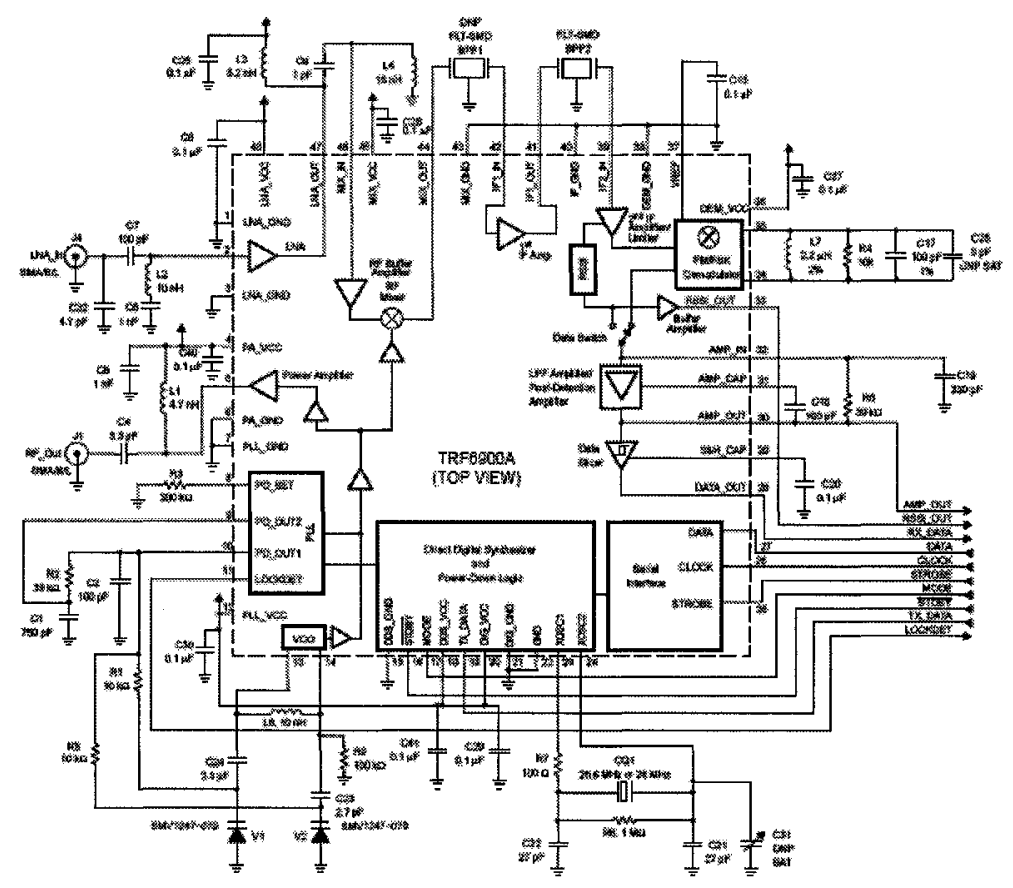

Figure 5: TRF6900A application circuit [51]. 
As Figure 5 shows, the TRF6900A requires the design of several circuits to support its operation: The loop filter for the Phase-Locked-Loop (PLL), the tank circuits for the Voltage-Controlled-Oscillator (VCO) and the demodulator, antenna matching circuitry, a number of IF (Intermediate Frequency) filters in the receive path, and an averaging filter for the data slicer. Information on each of those components can be found in [51].

Second-generation transceivers integrate the serdes block in addition to the RF modem. This frees the external baseband processor from the serialization/de-serialization tasks. Also, second-generation transceivers integrate much of the transceiver's supporting circuitry, thereby reducing the RF design complexity. Note however that an external baseband processor is still required to perform packet-handling. The CYWUSB6935 from Cypress Semiconductor [17] and the CC1010 from Texas Instruments [8] are examples of second-generation transceivers. The CYWUSB6935 is shown in the figure below. 


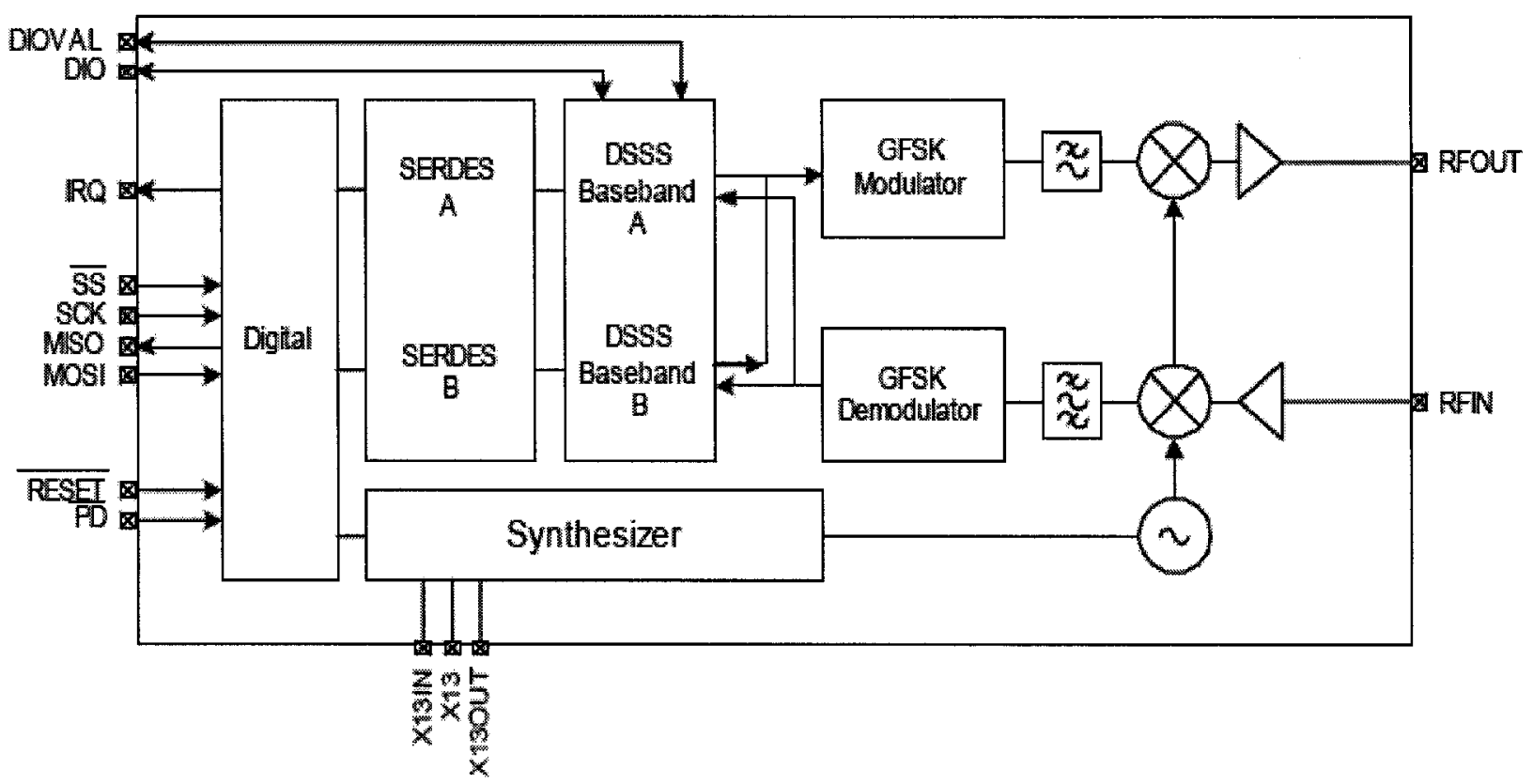

Figure 6: CYWUSB6935 is an example of a second-generation transceiver [17].

As can be seen in the figure, the transceiver contains a serdes block (SERDES A and B) in addition to the analog front-end. Implicit in the figure is the on-chip integrated supporting circuitry. RF design with the CYWUSB6935 reduces to designing the antenna matching circuitry. As can be seen above, RF design with a second-generation transceiver is much simpler than it is with a first-generation transceiver.

Third-generation transceivers integrate complete on-chip packet handlers. These packethandlers are responsible for packetizing data (adding preamble, addressing, CRC...etc.), and performing the bit/byte synchronization required for packet communication.

With third-generation transceivers, the baseband processor can be kept very simple. Data can be buffered in and out of the transceiver whenever available. The transceiver takes care of all other tasks required for packet-based wireless communication. The CC1100 
from Texas Instruments [9], the SX1211 from Semtech [48], and the SI4432 from Silicon Labs [26] are examples of third-generation transceivers. The CC1100 is shown in Figure 7 below.

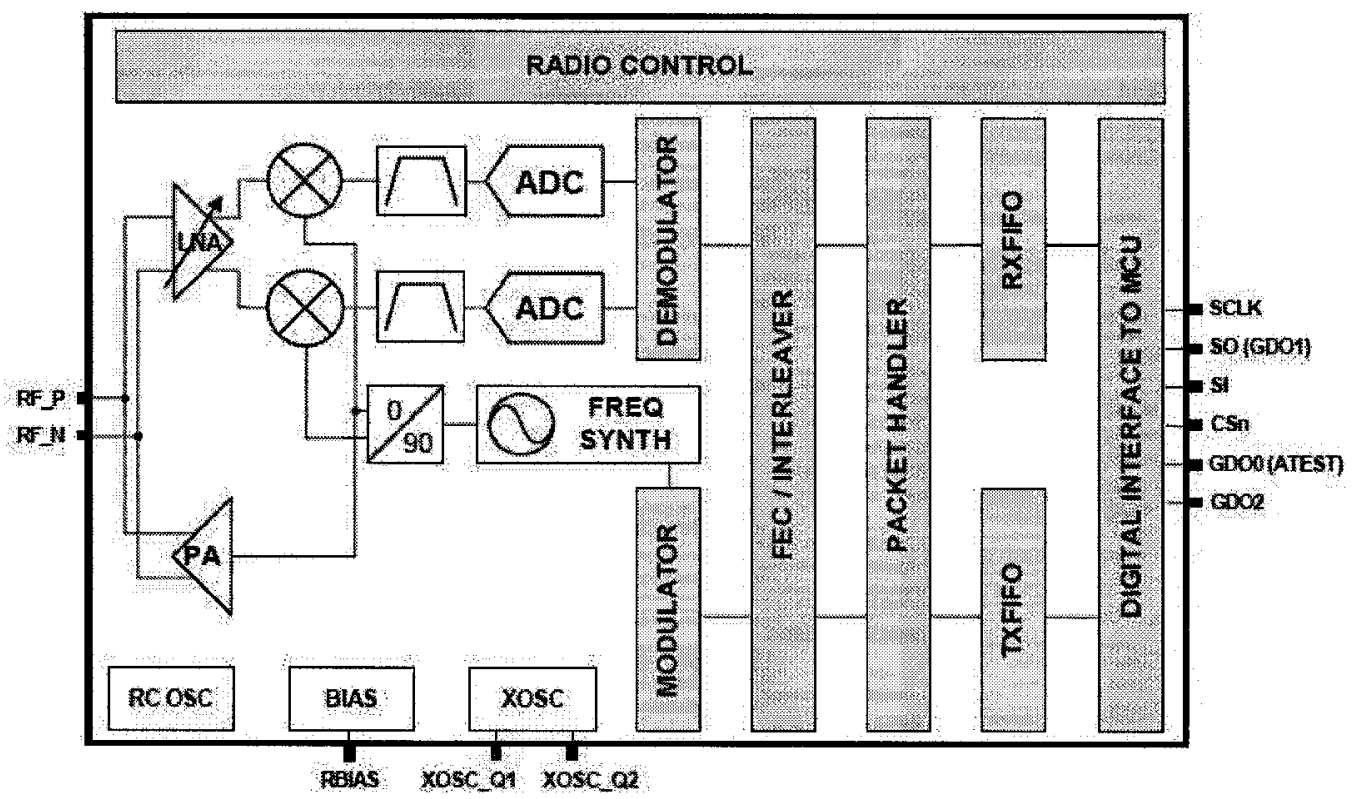

Figure 7: The CC1100 from TI is an example of a third-generation transceiver [9].

Fourth-generation transceivers integrate a complete microcontroller along with a complete third-generation transceiver, all on a single chip. The CC1110 from Texas Instruments [10] and the nRF24E1 from Nordic Semiconductor [37] are examples of fourth-generation transceivers.

The nRF24E1 for example, contains an 8051-compatible microcontroller and an nRF2401 third-generation transceiver [36]. The nRF24E1 is shown below in Figure 8. Fourth-generation transceivers can greatly reduce design cost and complexity since most wireless applications require an application processor and such transceivers happen to integrate one on-chip. 


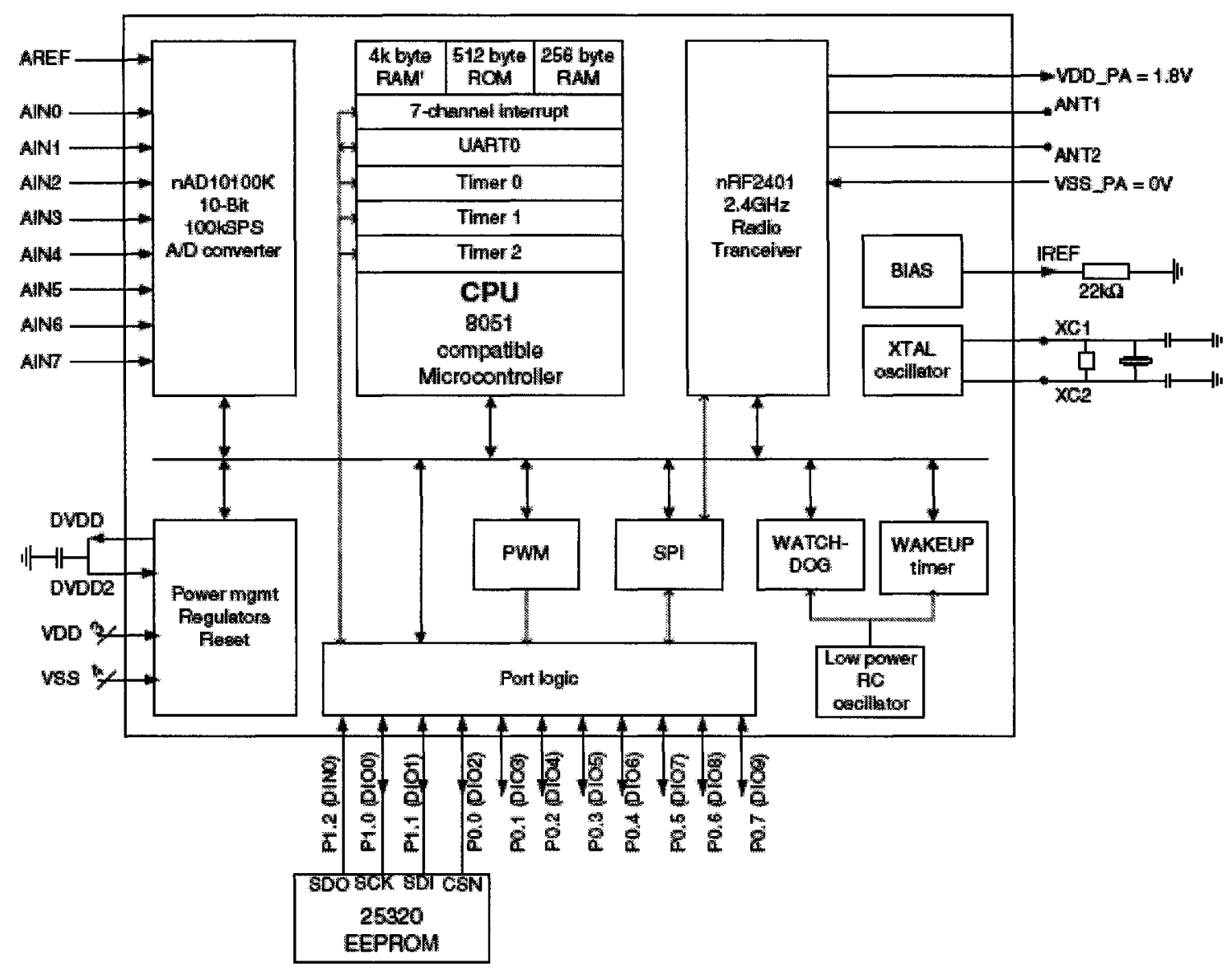

Figure 8: the nRF24E1 is an example of a fourth-generation transceiver [37].

Finally, fifth-generation transceivers are highly integrated SoC's which contain a number of digital blocks, of which a microcontroller and a transceiver form a small subset. The nRF24LU1 from Nordic Semiconductor [39] and the CC2511F32 from Texas Instruments [12] are examples of fifth-generation transceivers. The nRF24LU1 is shown in Figure 9 below. 


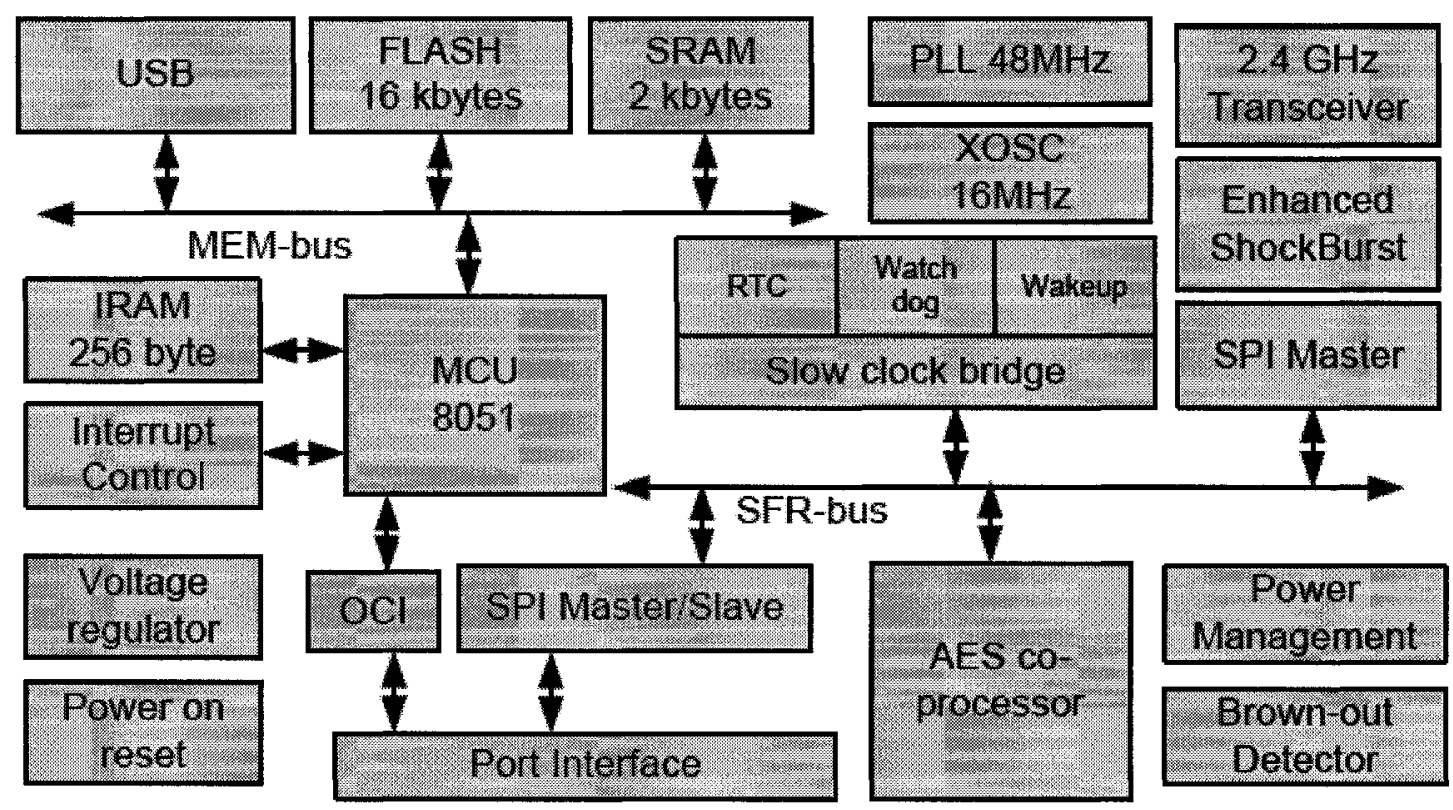

Figure 9: the nRF24LU1 SoC is an example of fifth-generation transceivers [39].

The nRF24LU1 SoC contains an 8051 microcontroller, an nRF24L01 [36] thirdgeneration wireless transceiver, a full-speed USB2.0 block, AES encryption co-processor, voltage regulator, an array of timers, a $48 \mathrm{MHz}$ PLL used for system timing, large amounts of RAM and ROM, and an array of communication interfaces, all integrated on a single chip. With such a SoC, one can design an application which for example reads data from a USB interface, encrypts it using the AES encryption engine, and transmits the encrypted data wirelessly using the $2.4 \mathrm{GHz}$ ISM transceiver.

Another interesting block found in this SoC is the Enhanced Shock-Burst block [36] which accompanies the wireless transceiver. This block implements an AutomaticRepeat-Request (ARQ) protocol. ARQ is a stop-and-wait protocol. When asked to transmit a packet, the block sends the packet across the wireless link and then waits for an acknowledgement to be received. If the acknowledgement is not received within a 
specified amount of time, the packet is re-sent again and again, upto a programmed number of times, thereby increasing the reliability of the link.

\subsection{Available Interference-Resistance Techniques}

The license-free nature of the ISM band implies that there's no control over the interference patterns in the band. In such an un-controlled environment, interference patterns can be described as unpredictable and services offered as best-effort [47].

This thesis is mainly concerned with the performance of a radio control application operating in the $2.4 \mathrm{GHz}$ ISM band. As presented later in Chapter 3, the performance metric of interest depends on the interarrival time of correctly received packets. The reliability of the wireless link between the transmitter and the receiver therefore has a direct effect on the performance of the radio control system. In practice, techniques such as Forward-Error Correction [7] (FEC) and Spread-Spectrum (SS) [45] are used to mitigate the effect of noise or interference present in the communication link.

Alongside the packet-handling and extensive integration features available in ISM transceivers, there exist a number of noise and interference-resistance techniques that can be employed by the transceiver to improve the reliability of the communication link. What follows is a survey of the noise and interference-resistance techniques available in ISM transceivers.

In practice, resistance to noise and interference can be achieved by: 1) maximizing the output power of the transmitter, 2) minimizing the sensitivity of the receiver, 3) and/or by 
introducing channel coding techniques such as Forward-Error-Correction (FEC) to improve the reliability of the link. Moreover, Spread Spectrum (SS) techniques such as Frequency Hopping (FHSS) and Direct Sequence (DSSS) can also be used to suppress interference [46].

ISM transceivers offer a variety of techniques to battle noise and interference in the ISM band. For example, the CC2500 from Texas Instruments [11] implements a 1/2 ForwardError-Correction (FEC) convolutional coding engine capable of providing up to $3 \mathrm{~dB}$ of coding gain [44]. The CYRF6936 from Cypress Semiconductor implements a configurable DSSS spreader and de-spreader capable of providing up to $10 \mathrm{~dB}$ of processing gain [16]. Moreover, most transceivers contain programmable frequency synthesizers with fast-settling Phase-Locked Loops (PLL) which enable frequency hopping applications [40].

In terms of output power, a multitude of RF amplifiers designed specifically for operation in the ISM band are available for use in applications where maximum output power is desired. Such RF amplifiers include the RMPA2455 2.4GHz power amplifier [23] and the BGA6589 $900 \mathrm{MHz}$ power amplifier [42]. An application circuit showing a typical interface between a transceiver and a power amplifier is shown below in Figure 10 [35]. 


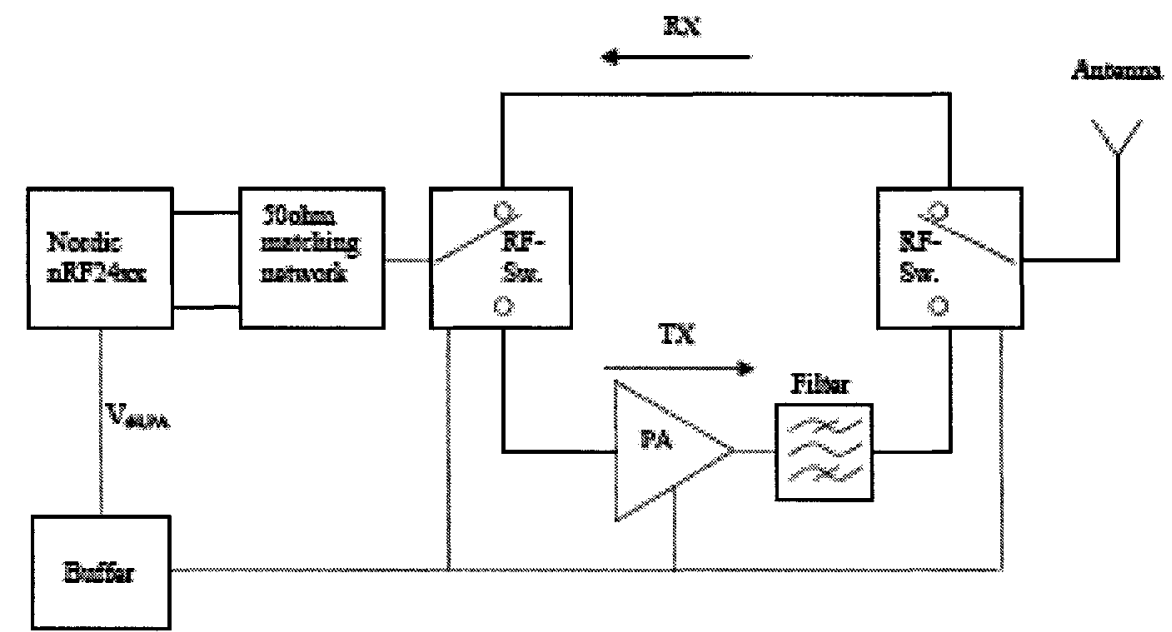

Figure 10: Typical interface between transceiver and power amplifier [35].

Receiver sensitivity depends on the signal bandwidth, receiver-generated noise, and minimum SNR required to sustain communication for a given modulation scheme [30]. The AT86RF230 [6] ZigBee-compliant transceiver from Atmel is an example of a transceiver with low sensitivity, listed at $-101 \mathrm{dBm}$ at a $P E R$ of $1 \%$ and data rate of $250 \mathrm{kbps}$. This is $10 \mathrm{~dB}$ to $12 \mathrm{~dB}$ better than what some of the other ISM transceivers offer at the same data rate $[11][16]$.

The maximum output power and the receiver sensitivity are generally fixed for a given transceiver. Other techniques such as FEC, DSSS and FHSS are used to further improve the communication link. Of those three techniques, FEC and DSSS are optimal only to the extent with which the transceiver that implements them allows. For example, the CYWUSB6935 implements a spreading engine which allows a maximum spreading factor of 64 chips per bit [17]. Similarly, the CC2500 implements a fixed 1/2 convolutional coding engine which does not allow any enhancements past the $1 / 2$ code rate limit [44]. It is therefore impossible to utilize the entire available bandwidth to resist 
noise and interference using FEC and/or DSSS alone, simply because the hardware does not allow it. Frequency hopping offers a solution to this problem.

ISM transceivers are designed to support Frequency Division Multiple Access (FDMA). The FDMA model splits the band into a large number of channels. In such a model, frequency hopping becomes the only means available that allows the utilization of the entire band for interference-resistance. Therefore one can make the following conclusion: The use of ISM transceivers in the design of $R C$ applications necessitates the use of frequency hopping if the application is to maximize interference-resistance.

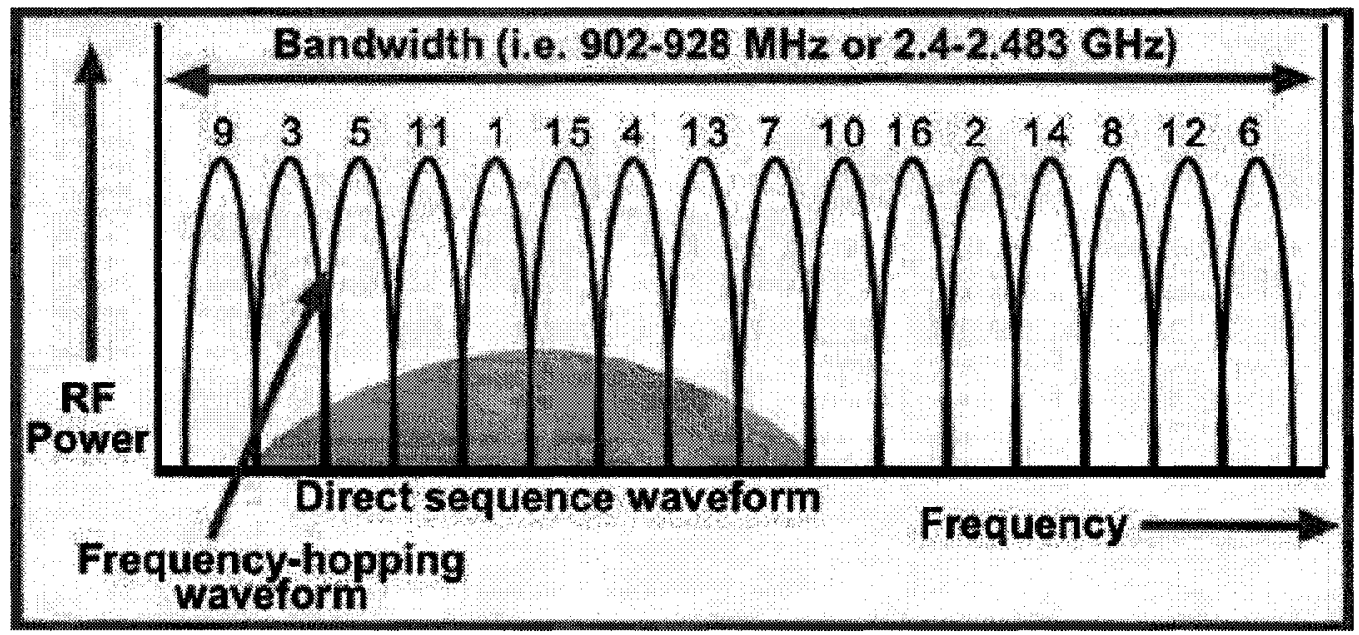

Figure 11: ISM band divided into small frequency channels [54]

Note that the necessity for frequency hopping is a result of the hardware limitations placed on the other noise and interference resistance techniques, not because it is optimal or because it performs better. Also, the use of frequency hopping as implied here does not prevent the use of other techniques within each channel. For example, a hybrid spread spectrum system can be implemented where frequency hopping is used to cover all the 
available channels, but where within each channel direct sequence can be used to further mitigate interference effects. Several performance studies on hybrid spread spectrum systems can be found in the literature, including [59][60][61].

This chapter presented the state of the art in ISM transceiver technology. It looked at available interference-resistance techniques and concluded that the use of ISM transceivers necessitates the use of frequency hopping to maximize interferenceresistance.

As will be shown in the following chapter, a number of variables are present in the design of a frequency hopping real-time radio control system. A performance metric is needed to differentiate one implementation from another, and enable the selection of the optimal one. To that end, the next chapter derives a performance metric based on the system latency of frequency hopping radio control systems. 


\section{Performance Metric for System Latency}

Real-time radio control requires that the system responds to user inputs with as little latency as possible. Real-time operation requires instantaneous response. Imperfections in the wireless channel introduce communication errors which causes random latency in the response of the system. The time it takes the system to respond to a user input is here referred to as the system latency. System latency is mainly dependant on the inter-arrival time of control information from the transmitter to the receiver. Therefore, the robustness of the wireless link established between the transmitter and the receiver has a major effect on the system latency.

A lag is here defined as an event which occurs when the system latency exceeds a specified real-time threshold. In applications where input is being applied by a user (human), the real-time threshold becomes the Human Response Time $(H R T)$ [25]. HRT is a time threshold above which the user notices a lag, but below which the user perceives response as instantaneous. $H R T$ shows up in other real-time applications that interface with users such as Voice-over-IP (VoIP). It is generally estimated to be around 100ms. The user experience can be defined in terms of the probability of lag occurrence during a radio control session. I define the System Lag Occurrence Probability (SLOP), as the probability of a lag occurring in the output of a real-time radio control system. Section 3.1 derives $S L O P$ for a single channel (non-hopping) radio control system. 
It was concluded in the previous chapter that a practical radio control system operating in the ISM band requires the use of frequency hopping to maximize its interference resistance. Therefore to make the thesis practical, Section 3.2 derives SLOP for a frequency hopping radio control system.

The communication system dealt with in this thesis is packet-based. As will be shown later, the main decision criterion used in the tracking and acquisition engines is based on the state of packet reception (correct or corrupt). The derivation of $S L O P$ in the next two sections therefore places emphasis on the average Packet-Error Rate $(P E R)$ parameter. I believe that PER is a more suitable parameter to use in this thesis than say the Bit-Error Rate $(B E R)$ or Signal-to-Interference-and-Noise Ratio (SINR) of the channel. The use of PER abstracts away the details of the underlying wireless channel and keeps the focus on the application layer design instead, as intended from the beginning.

The performance of frequency hopping systems under the presence of different types of interference is studied extensively in the literature. For example, [62] and [63] look at wideband and partial-band noise jamming. [64] looks at follower partial-band jamming, while [65] looks at partial-band multi-tone jamming. In this thesis however, the wireless channel model, the modulation type used, and the type of interferers present in the band are all abstracted in the average $P E R$ parameter. The average $P E R$ of a frequency hopping system is here calculated as:

$$
P E R=\frac{1}{q} \sum_{x=1}^{q} P E R_{x}
$$


where $P E R_{x}$ is the $P E R$ of channel $x$ and $q$ is the total number of channels. This has the effect of averaging out any interference present in the band across all the channels. This model is equivalent to assuming the source of interference to be wideband Gaussian noise power [7]. The simulator built in Chapter 4 as well as the implementation presented in Chapter 5 assume a similar model of operation in which the channel is reduced to an increasing (or decreasing) levels of Gaussian noise. Studying the effect of different physical layer models on SLOP is left for future work.

\subsection{Single-Channel SLOP}

In theory, a lag occurs when the interarrival time between two correctly-received control packets exceeds $H R T$. SLOP can then be defined as the probability that the interarrival time between two correctly-received control packets exceeds $H R T$. Assuming that packet errors in a single-channel are statistically independent, and that each packet contains all of the control commands needed by the system to drive its outputs, the SLOP of a singlechannel (non-hopping) system can be easily derived as:

$$
S L O P_{s c}=P E R^{P}
$$

where $S L O P_{s c}$ is the SLOP of single-channel system, PER is the packet error rate of the channel, and $P$ is the number of packets transmitted per HRT. In other words, a lag occurs in a single-channel system when $P$ or more consecutive packets are corrupted by the channel, and the probability that such an event happens is given by the above equation.

\subsection{Frequency-Hopping $S L O P$}

The drawback in single-channel radio control systems is that communication is extremely sensitive to co-channel (same-channel) interference, here referred to as blocking. A 
frequency hopping system fights blocking by dispersing the blocker's effect across all of the channels used in hopping [46].

In a frequency hopping application, the frequency band used for communication is divided into a number of smaller bins called channels. To communicate the transmitterreceiver pair "hops" on to one channel and exchanges a packet of information, then "hops" onto another channel and exchanges another packet of information. This continues until the communication session is completed.

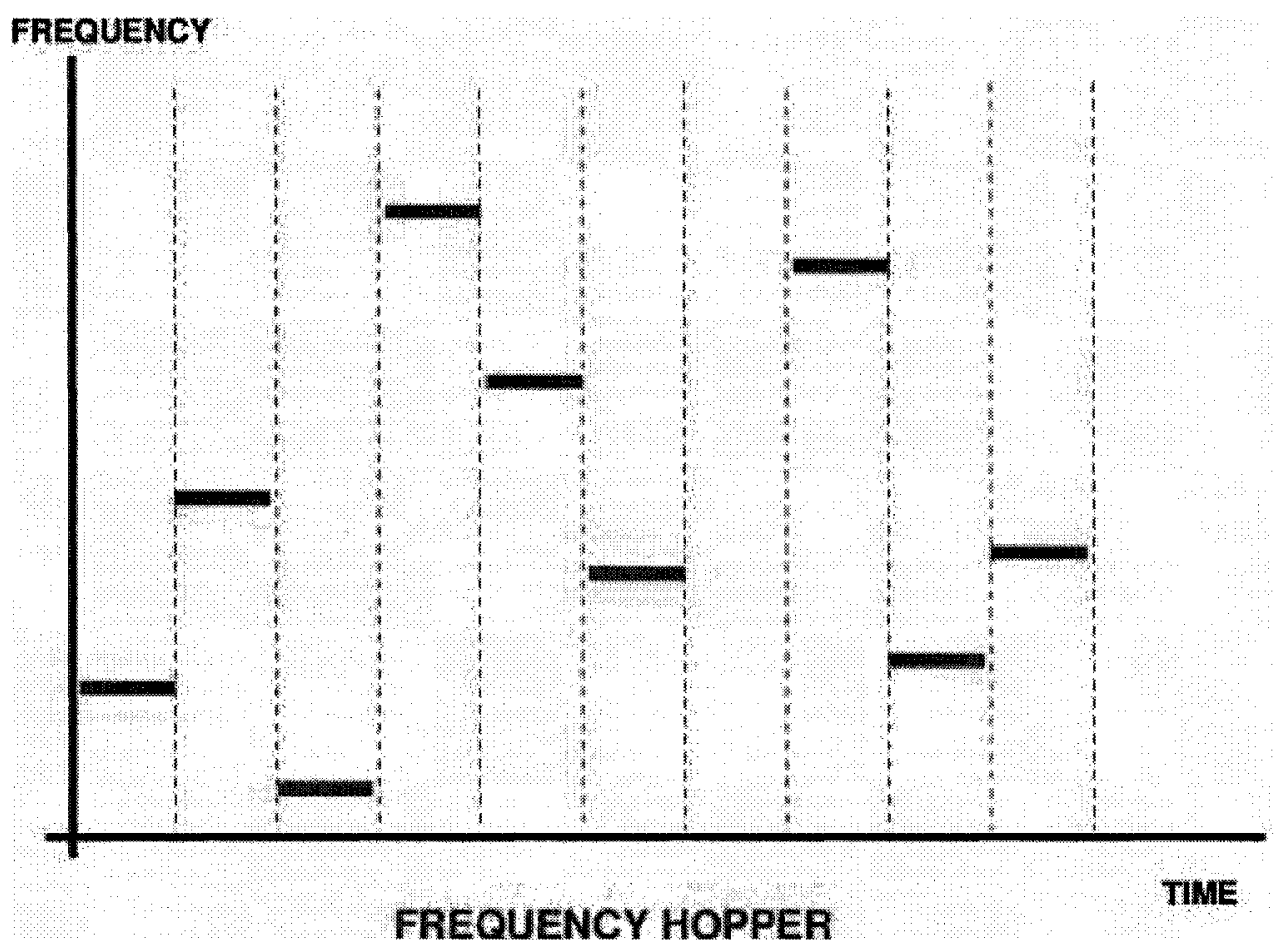

Figure 12: Frequency hopping [53]

There needs to be some form of synchronization between the transmitter and the receiver to keep them synchronized in both frequency and time. Two synchronization processes are required: coarse synchronization and fine synchronization [7]. 
Coarse synchronization is the process of establishing an initial communication link between the transmitter-receiver pair. This process is more commonly referred to as acquisition, since the transmitter and the receiver try to acquire lock on each other.

During the acquisition process, a search algorithm is performed in both frequency and time. The search algorithm aims at (re)-synchronizing the transmitter-receiver pair as quickly as possible. This search algorithm can be implemented either at the transmitter, or at the receiver, or both. The average time it takes the transmitter-receiver pair to perform the search and acquire lock on each other is called the acquisition time ( $\left.T_{a c q}\right)$.

Once lock between the transmitter and the receiver is acquired, fine synchronization is required to keep the pair in-lock, and therefore synchronized, with each other for as long as possible. Fine synchronization is more commonly referred to as tracking, since the transmitter-receiver pair try to track each other and remain in-lock for as long as possible. The average time during which the pair remains in-lock is called the lock-in time $\left(T_{\text {lock }}\right)$.

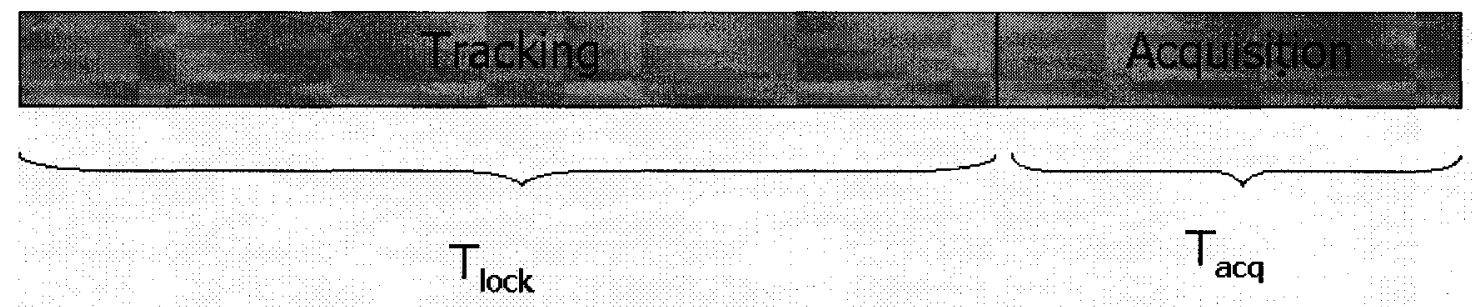

Figure 13: Average lock-in and acquisition times of a frequency hopping system 
On the average, a frequency hopping system will periodically switch between acquisition and tracking. During tracking, the only source of performance degradation is the channel imperfections (i.e. noise and interference). Performance degradation here refers to an increase in the system latency (response).

During acquisition on the other hand, an additional source of performance degradation exists: missed packets. Missed packets arise when the transmitter and the receiver are resident on different channels, causing packets transmitted to be completely missed by the receiver.

The acquisition process in frequency hopping systems can consume relatively large amounts of time [27]. This increases the likelihood of lag occurrence in the system. The derivation of a frequency hopping $S L O P$ enables the comparison of different frequency hopping implementations, and therefore aids in the selection of optimal design parameters for a radio control system.

To begin the derivation of frequency hopping SLOP, I'll first look at the interaction between acquisition and tracking modes during a communication session. Initially the transmitter-receiver pair is out-of-lock and therefore acquisition is first initiated. The process responsible for performing acquisition is here referred to as the acquisition engine. 
After some time $T_{a c q}$ the transmitter-receiver pair acquire lock on each other, acquisition is terminated, and tracking is initiated. The process responsible for performing tracking is here referred to as the tracking engine.

During tracking, the transmitter-receiver pair will try to remain in-lock with each other for as long as possible. After some time $T_{\text {lock}}$, various factors such as interference and time/frequency drift cause the transmitter-receiver pair to loose lock of each other and reinitiate acquisition [55]. The time it takes the tracking engine to conclude that it has lost lock is called $T_{L A}$ (i.e. Lock $\rightarrow$ Acquisition). Depending on $T_{L A}$, Two separate cases for frequency hopping SLOP could be derived. Section 3.2.1 derives SLOP for the case where $\mathrm{T}_{\mathrm{LA}}$ is greater than $H R T$. Section 3.2.2 derives $S L O P$ for the case where $\mathrm{T}_{\mathrm{LA}}$ is less than or equal to $H R T$.

\subsubsection{Case 1: Zero-Time Acquisition $\left(T_{L A}>H R T\right)$}

In case 1 , the time it takes the tracking engine to conclude that it has lost lock exceeds $H R T$. Therefore in case 1, a lag occurs before acquisition is initiated. Assuming that errors between consecutive packets are statistically independent, and that one packet is transmitted per hop, SLOP for case 1 becomes:

$$
S L O P=P E R^{H R T / T_{\text {dwell }}}
$$

where $T_{d w e l l}$ is the channel dwell time (hopping period). For a given PER, a case $1 S L O P$ can be minimized by minimizing $T_{\text {dwell }}$. Minimum $T_{\text {dwell }}$ is equal to the time it takes the transmitter to hop to a new channel (PLL settling time $T_{\text {settle }}$ ) plus the time it takes to transmit a packet (packet transmission time $T_{\text {packet }}$ ): 


$$
T_{d \text { well }}=T_{\text {settle }}+T_{\text {packet }}=T_{\text {settle }}+\frac{b}{R}
$$

Where $b$ is the packet length in bits, and $R$ is the data rate. The PLL settling time $\left(T_{\text {settle }}\right)$ and the size of packet $(b)$ are usually fixed for a given implementation. Usually the data rate $R$ is varied to minimize the dwell time ( $\left.T_{d w e l l}\right)$ and therefore minimize SLOP.

\subsubsection{Case 2: Non-Zero-Time Acquisition $\left(T_{L A} \leq H R T\right)$}

In case 2 the time it takes the lock detector to conclude that it has lost lock is less than $H R T$. This means that it is upto the acquisition engine to try to acquire lock in the time remaining for an $H R T$ to elapse. Otherwise a lag occurs.

A case $2 S L O P$ is the probability of two events happening: 1) the event that the system enters acquisition mode and 2) the event that the system fails to acquire in the time remaining for an $H R T$ to elapse:

$$
S L O P=P \text { (going into acquisition } \cap \text { failing to acquire before HRT elapses) }
$$

Assuming that the tracking and acquisition processes are statistically independent, SLOP becomes:

$$
S L O P=P_{L A} P\left(T>\left(H R T-T_{L A}\right)\right)
$$

Where $P_{L A}$ is the probability of loosing lock, $T_{L A}$ is the time it takes the tracking engine to conclude that it has lost lock, and $T$ is the time it takes the acquisition engine to re-acquire lock. 
$P_{L A}$ and $T_{L A}$ depend on the algorithm used for tracking, while the probability of successful acquisition depends on the algorithm used for acquisition. Therefore to complete the derivation of a case $2 S L O P$, I must first analyze the acquisition and tracking engines in finer detail.

As will be presented shortly, many different tracking and acquisition engines have been studied in the literature. In general, the goal of the tracking engine is to maximize the lock-in time, while the goal of the acquisition engine is to minimize the acquisition time. Bi-directional tracking and acquisition schemes can aid in achieving those goals. For example, the Adaptive Frequency Hopping (AFH) schemes proposed in [66] and [67] aim at improving the overall performance of frequency hopping systems. While studying the effect of such schemes on the performance of radio control systems is preferable, it is left for future work.

In this thesis, I decided to use the $\mathrm{N}$-state lock detector tracking engine and the uniform serial acquisition engine to base the rest of the analysis on. I selected these two engines for three main reasons:

1. They both simplify analysis.

2. They both simplify design and implementation.

3. They both can be designed to satisfy the independence condition required for the derivation of SLOP (shown above). 


\subsubsection{Tracking Strategies}

Various tracking strategies have been studied in literature, the most common being TauDither [45], Delay-Lock [4], and Split-Bit Tracking loops. For packet-based systems, Modified Transmitted Reference [22] techniques seem to be more adequate as they are simpler to implement in firmware.

A Modified Transmitted-Reference tracking engine is more commonly referred to as an N-state lock detector [46]. This section derives $P_{L A}$ and $T_{L A}$ for an N-state lock detector. The basic principle of operation is shown in Figure 14.

The N-state lock detector contains $N$ states, named Lock_O to Lock_(N-1). Operation in one of those $\mathrm{N}$ states represents operation in tracking mode. State Lock_O represents correct packet reception during tracking mode, while states from Lock_l to Lock_(N-1) represent corrupted packet reception during tracking mode. An additional state, called Acq, represents operation in the acquisition mode and is shown to the right of Figure 14.

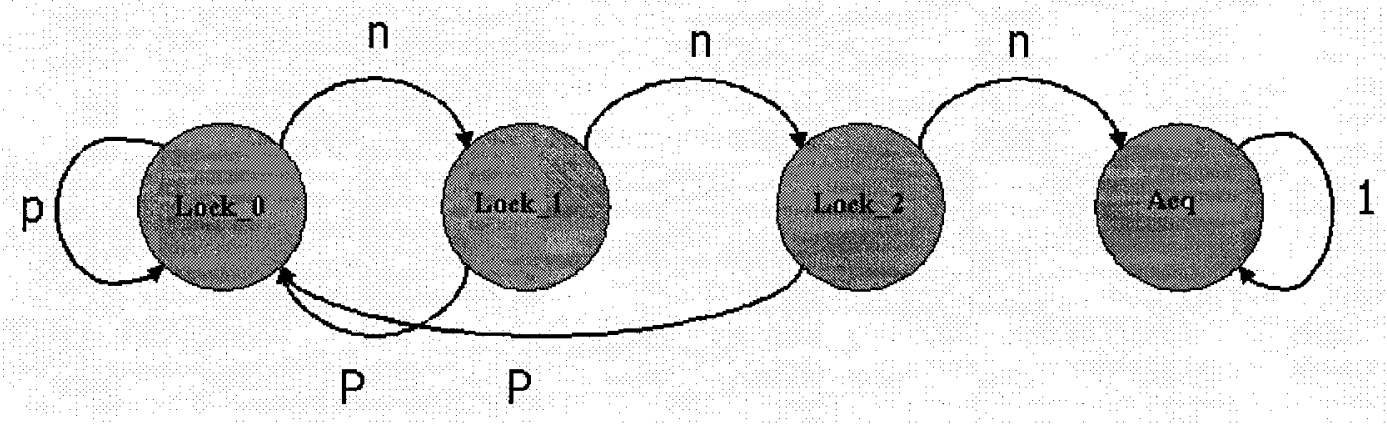

Figure 14: State transitions in an $\mathrm{N}$-State lock detector. $\mathrm{N}=3$

The N-state lock detector begins operation in state Lock_0, where the transmitter-receiver pair is in lock and is exchanging packets correctly. During each hop, the transmitter- 
receiver pair exchanges a new packet. If the packet is received correctly, the lock detector generates a positive decision and moves to (or remains in) state Lock_0. If the packet is received corrupted, the lock detector generates a negative decision and moves one state to the right, towards state $A c q$.

This process is repeated at each of the states of the lock detector. If $\mathrm{N}$ consecutive negative decisions are generated, the lock detector terminates tracking and initiates acquisition. Positive decisions occur with probability $p$. Negative decisions occur with probability $n$.

To simplify the analysis of $P_{L A}$ and $T_{L A}$, I assume that tracking is maintained when one complete packet is exchanged correctly. This has the following effects:

$$
n=P E R \quad \text { and } \quad p=1-P E R
$$

As mentioned previously, $P_{L A}$ is the probability of the tracking engine concluding that it has lost lock. In other words, $P_{L A}$ is the probability of terminating tracking and initiating acquisition. With an N-state lock detector, $P_{L A}$ becomes the probability of exchanging $\mathrm{N}$ consecutive corrupted packets. Assuming that errors between packets are statistically independent, $\mathrm{P}_{\mathrm{LA}}$ becomes:

$$
P_{L A}=P E R^{N}
$$

$T_{L A}$ is the time it takes the tracking engine to conclude that it has lost lock. With an Nstate lock detector, $T_{L A}$ becomes the time it takes for the exchange of $\mathrm{N}$ consecutive corrupted packets:

$$
T_{L A}=N T_{d w e l l}
$$


The above derives $P_{L A}$ and $T_{L A}$ for an N-state lock detector. To complete the derivation of case 2 frequency hopping SLOP, I will derive the probability of successful acquisition. The probability of successful acquisition depends on the algorithm used in the acquisition engine.

\subsubsection{Acquisition Strategies}

Acquisition strategies have been studied extensively in literature, the most common being serial [3] and parallel [33]. Other acquisition techniques include message passing [34] and adaptive-antenna array [21].

Serial acquisition techniques try to acquire lock between the transmitter and the receiver by searching the channels serially (i.e. one after the other) until the correct channel is found [3]. Uniform serial acquisition treats all channels as equally possible candidates for successful acquisition. Non-uniform serial acquisition favors some channels over others during its search.

Parallel acquisition techniques acquire lock by examining all channels simultaneously [33]. In practice, this is achieved by employing a bank of correlators, each tuned to one of the channels.

Serial acquisition is simple to implement but is relatively slow in acquiring lock. Parallel acquisition is complex to implement but is fast in acquiring lock. In practice, a hybrid of both techniques is usually employed to reach a relatively simple solution with relatively 
fast acquisition times. To simplify the analysis, this section will derive the required parameters using a uniform serial acquisition engine.

The mean acquisition time and its standard deviation for a uniform serial acquisition engine were derived in [27] and [20]:

$$
\begin{gathered}
T_{a c q}=T_{\text {dwell }} \frac{2+\left(2-P_{D}\right)(q-1)\left(1+K P_{F A}\right)}{2 P_{D}} \\
\sigma_{a a q}^{2}=T_{\text {drel }}^{2}\left[\left(1+K P_{F A}\right)^{2} q^{2}\left(\frac{1}{12}-\frac{1}{P_{D}}+\frac{1}{P_{D}^{2}}\right)+6 q\left[K(K+1) P_{F A}\left(P_{D}-P_{D}^{2}\right)+\left(1+K P_{F A}\right)\left(4-2 P_{D}-P_{D}^{2}\right)\right]+\left[\frac{1-P_{D}}{P_{D}^{2}}\right]\right]
\end{gathered}
$$

where $T_{a c q}$ is the mean acquisition time, $\sigma_{a c q}$ is its standard deviation, $T_{d w e l l}$ is the dwell time, and $q$ is the number of channels.

The probability of detection $\left(P_{D}\right)$ is the probability of correctly terminating acquisition when the correct channel is being probed. The probability of a false alarm $\left(P_{F A}\right)$ is the probability of erroneously terminating acquisition when an incorrect channel is being probed. Such an event is called a false alarm, and has a penalty $K$ associated with it. $K$ is the number of hops it takes the tracking engine to conclude that lock was falsely acquired and therefore re-initiate acquisition. Low Signal-to-Noise Ratio $(S N R)$ and high channel distortion (fading and Inter-Symbol Interference $I S I$ ) drive $P_{D}$ to 0 and $P_{F A}$ to 1 . 
To simplify the analysis, I assume that acquisition ends when one complete packet is received correctly, which is a fair assumption in a packet-based communication system. This has the following effects:

$$
P_{D}=1-P E R \quad \text { and } \quad P_{F A}=0
$$

$P_{F A}$ becomes negligible since it will be unlikely that an entire correct packet is falsely detected. Furthermore, assuming that $q>>1$ and $K<<q$, which are fair assumptions in practice, $T_{a c q}$ and $\sigma_{a c q}$ simplify to the following:

$$
\begin{gathered}
T_{a c q}=q T_{d w e l l}\left[\frac{2-P_{D}}{2 P_{D}}\right] \\
\sigma_{a c q}=q T_{d w e l l} \sqrt{\left[\frac{1}{12}-\frac{1}{P_{D}}+\frac{1}{P_{D}^{2}}\right]}
\end{gathered}
$$

It was shown that if I assume that the tracking and acquisition processes are statistically independent, SLOP becomes:

$$
S L O P=P_{L A} P\left(T>\left(H R T-T_{L A}\right)\right)
$$

where $P_{L A}$ is the probability of loosing lock, $T_{L A}$ is the time it takes the tracking engine to conclude that it has lost lock, and $T$ is the time it takes the acquisition engine to re-acquire lock. Attempts to derive the Cumulative Distribution Function (cdf) and the Probability Density Function (pdf) of $\mathrm{T}$ were made in [68] and [69]. The model of the acquisition engine studied in the literature is complex however. False alarms, as well as other variables in the system tend to complicate the derivation of a proper pdf or cdf.

In this thesis, we consider packet-based system operation, in which all decisions made in the acquisition engine are based on the state of packet reception during every hop interval: 
Either correct packet reception or corrupt packet reception. In such a model, the probability of a false alarm becomes negligible. Essentially, every hop interval becomes a new Bernoulli trial. If a correct packet is received, the trial is declared a success and the acquisition process is terminated. Otherwise the trial is declared a failure and the acquisition process continues.

This reduces the acquisition time random variable to be defined simply as the number of consecutive failed Bernoulli trials made until a successful trial is made. By definition, $T$ therefore becomes a geometric random variable [1]. The limiting form of $T$ as the number of trials goes to infinity is the exponential random variable. Therefore, if I model the acquisition time $T$ as an exponential random variable, $S L O P$ becomes:

$$
S L O P=P_{L A}\left(1-F_{T}\left(H R T-T_{L A}\right)\right)
$$

where $F_{X}(x)$ is the cumulative distribution function of the exponential random variable $X$ with parameter $\lambda=\frac{1}{E[X]} \cdot F_{X}(x)$ is defined as $[1]$ :

$$
F_{X}(x)=1-e^{-\lambda x} \quad x \geq 0
$$

where in this case,

$$
\lambda=\frac{1}{T_{a c q}} \quad \text { and } \quad x=H R T-T_{L A}
$$

but it was shown that:

$$
T_{L A}=N T_{d w e l l}
$$

and,

$$
P_{L A}=P E R^{N}
$$

therefore a case 2 frequency hopping SLOP becomes: 


$$
S L O P=P E R^{N}\left(1-F_{T}\left(H R T-N T_{d w e l l}\right)\right)
$$

where,

$$
T_{a c q}=\frac{1}{\lambda}=q T_{d w e l l}\left[\frac{2-P_{D}}{2 P_{D}}\right]
$$

Simulation and implementation results presented in Chapters 4 and 5 validate modeling the acquisition time as an exponential random variable. Further validation can be made by running a simulation experiment to collect a large number of acquisition time samples and plotting their probability density function (pdf). This type of validation is left for future work. 


\section{Simulation Setup and Results}

The purpose of this chapter is to verify the results derived in Section 3.2 and to study the effect of various design variables on the performance of a frequency hopping real-time radio control system.

There are two sections to this chapter. Section 4.1 describes the simulator that was built to study the effects of the different design variables on the performance of the system. Section 4.2 presents and discusses the simulation results.

\subsection{Packet-Based Frequency Hopping Simulator}

The simulator is built using Java [50]. It consists of a transmitter, a receiver, and a range of 20 to 80 frequency channels. Each channel is characterized with a Bit Error Rate $(B E R)$. Using its $B E R$, each channel computes the corresponding Packet Error Rate (PER) according to the following formula [28]:

$$
P E R=1-(1-B E R)^{b}
$$

where $b$ is the length of a packet in bits.

Each channel contains a random number generator which corrupts packets at a rate equal to its computed PER. There are two types of channels in the simulator: Blocked and nonblocked. Blocked channels are ones with PER equal to 100\%. Blocked channels are used to simulate the effect of powerful co-channel (same-channel) interferers. Non-blocked channels are ones with PER equal to $15 \%$. A PER of $15 \%$ corresponds to a BER of $1 \mathrm{e}-3$ 
and a packet length of 160 bits. These are typical values used in packet-based wireless communication systems with AWGN channels.

The transmitter hops pseudorandomly across all the channels and generates a single packet for each hop. The hopping pattern is uniform. Each generated packet is passed to the channel at which the transmitter currently resides. The channel in turn either corrupts the packet or passes it to the receiver uncorrupted, depending on the PER of the channel.

The receiver implements uniform serial acquisition and N-State lock detection. The Finite State Machine (FSM) of the receiver is shown in Figure 15. The acquisition engine consists of a single state called $A C Q$. The lock detector consists of two states called $L O C K \_0$ and $L O C K \_N$. The receiver FSM defines the interaction between these three states.

The simulator runs by generating a programmed number of timer ticks. At each tick, the transmitter hops to a new channel and transmits a packet at that channel. The channel corrupts the packet or passes it to the receiver un-corrupted depending on the $P E R$ of the channel. Two new inputs are therefore available to the receiver with each new tick:

1. The newly transmitted packet (either corrupted or un-corrupted),

2. And the channel at which the new transmission occurred. 


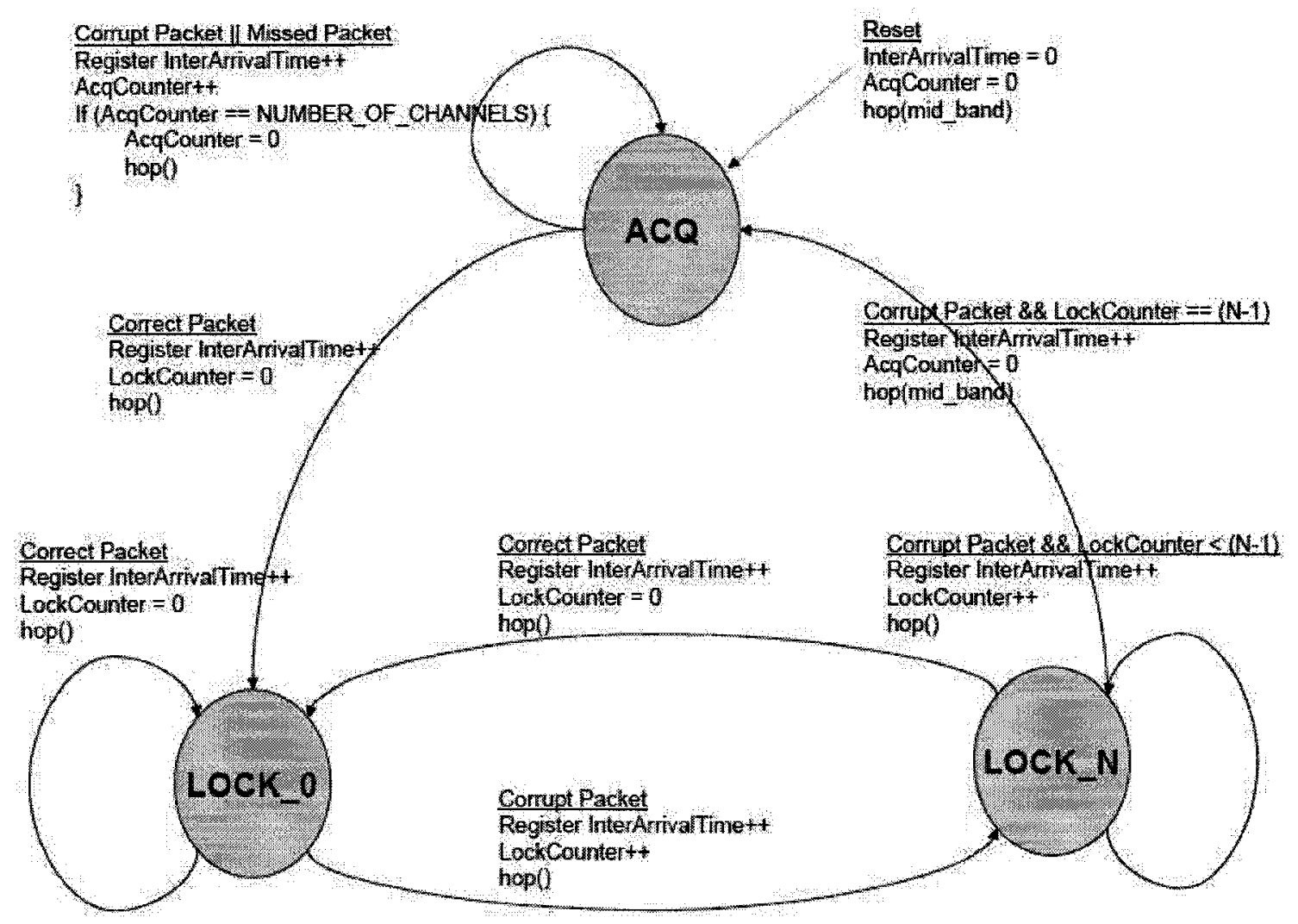

Figure 15: Receiver FSM

The receiver generates one of three events depending on the two inputs from the simulator:

1. Non-Corrupted-Packet-Received Event is the event that the receiver's current channel is equal to the transmitter's current channel and the received packet was not corrupted by the channel.

2. Corrupted-Packet-Received Event is the event that the receiver's current channel is equal to the transmitter's current channel and the received packet was corrupted by the channel. 
3. Missed-Packet Event is the event that the receiver's current channel is not equal to the transmitter's current channel and therefore the transmitted packet was entirely missed, regardless of the state of the packet itself (corrupted or uncorrupted). This event can only occur during the acquisition state $(A C Q)$ since this is the only state during which the transmitter and the receiver can be out-of-lock.

The inter-arrival time of correctly received packets is measured in ticks (time normalized to the channel dwell time) and is logged with every new tick. Non-Corrupted-PacketReceived events reset the inter-arrival time, while Corrupted Packet Received events and Missed Packet events increment the inter-arrival time. The inter-arrival time of each packet during a control session is plotted and analyzed against different design variables for performance evaluation.

A lag is registered every time the inter-arrival time exceeds $H R T$. The reception of a correct packet represents an opportunity for a lag to occur. SLOP is computed at the end of a simulation session as the ratio of the number of lags to the number of correct packets received. SLOP is plotted and analyzed against different design variables for performance evaluation.

\subsection{Simulation Results}

Two sets of simulations were carried out. The first set plots $S L O P$ vs. $P_{D}$ and $S L O P$ vs. $N$. The purpose of the first set is to verify the theoretical results derived in chapter 3 . The second set plots the average inter-arrival time of correctly received packets against different design variables. The purpose of the second set is to study the effect of the 
different design variables on the performance of a packet-based frequency hopping system.

In chapter 3 , it was shown that if the acquisition time is modeled as an exponential random variable with parameter $\lambda$ then $S L O P$ can be derived as:

$$
S L O P=P E R^{N}\left(1-F_{T}\left(H R T-N T_{d w e l l}\right)\right)
$$

where $F_{X}(x)$ is the cumulative distribution function of the exponential random variable $X$ with parameter $\lambda$, and

$$
T_{a c q}=\frac{1}{\lambda}=q T_{d w e l l}\left[\frac{2-P_{D}}{2 P_{D}}\right]
$$

Therefore, $S L O P$ depends on the probability of detection $\left(P_{D}\right)$ and the number of states in an N-state lock detector $(N)$. In this section, this dependency is simulated and plotted along with the theoretical results to verify the derivations.

The first simulation plots $S L O P$ against the probability of detection $\left(P_{D}\right)$. Results are shown in Figure 16. Results show that the simulation is in perfect agreement with theory. This validates the derivations made in Section 3.2 and serves as evidence for correctly modeling the acquisition time as an exponential random variable.

The second simulation plots SLOP against the number of states in an $N$-state lock detector $(N)$. Results are shown in Figure 17. As can be seen in the figure, results show that the simulation is in perfect agreement with theory. This validates the derivations 
made in section 3.2 and serves as evidence for correctly modeling the acquisition time as an exponential random variable.

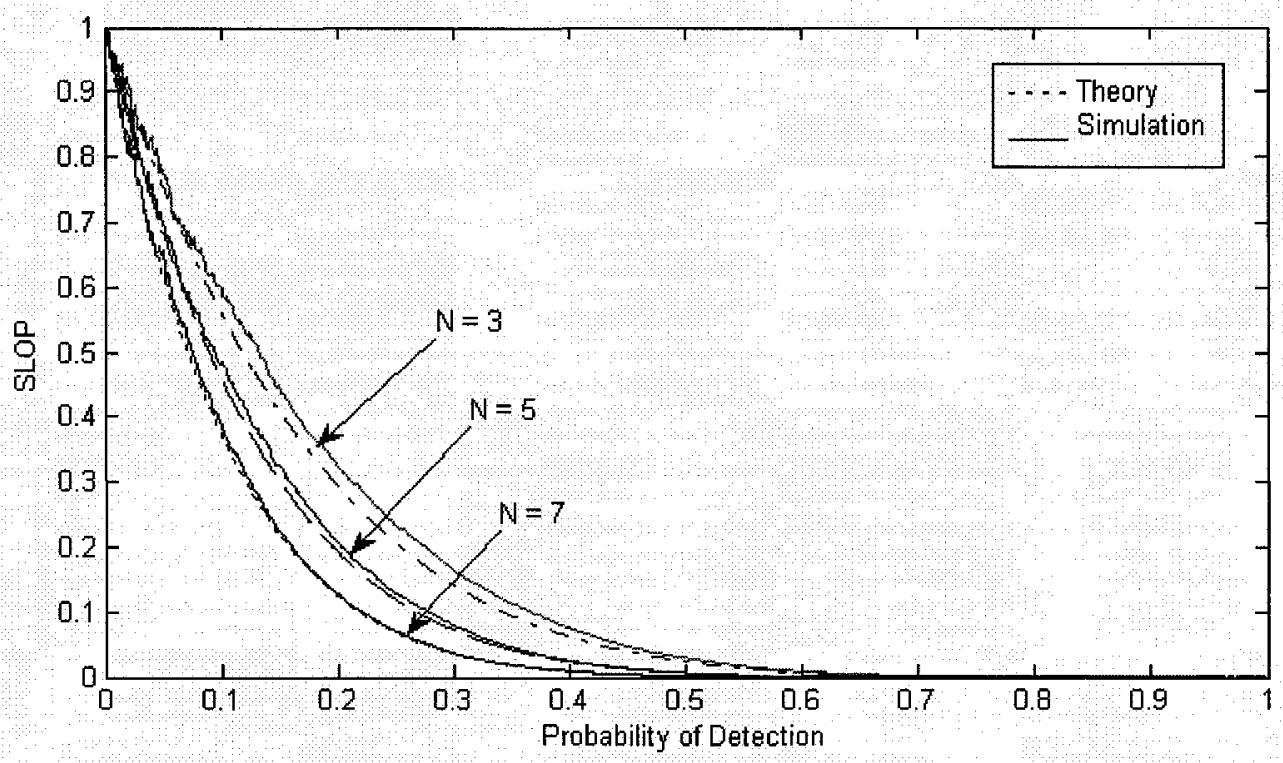

Figure 16: $S L O P$ vs. $P_{D}(q=40, H R T=100 \mathrm{~ms})$

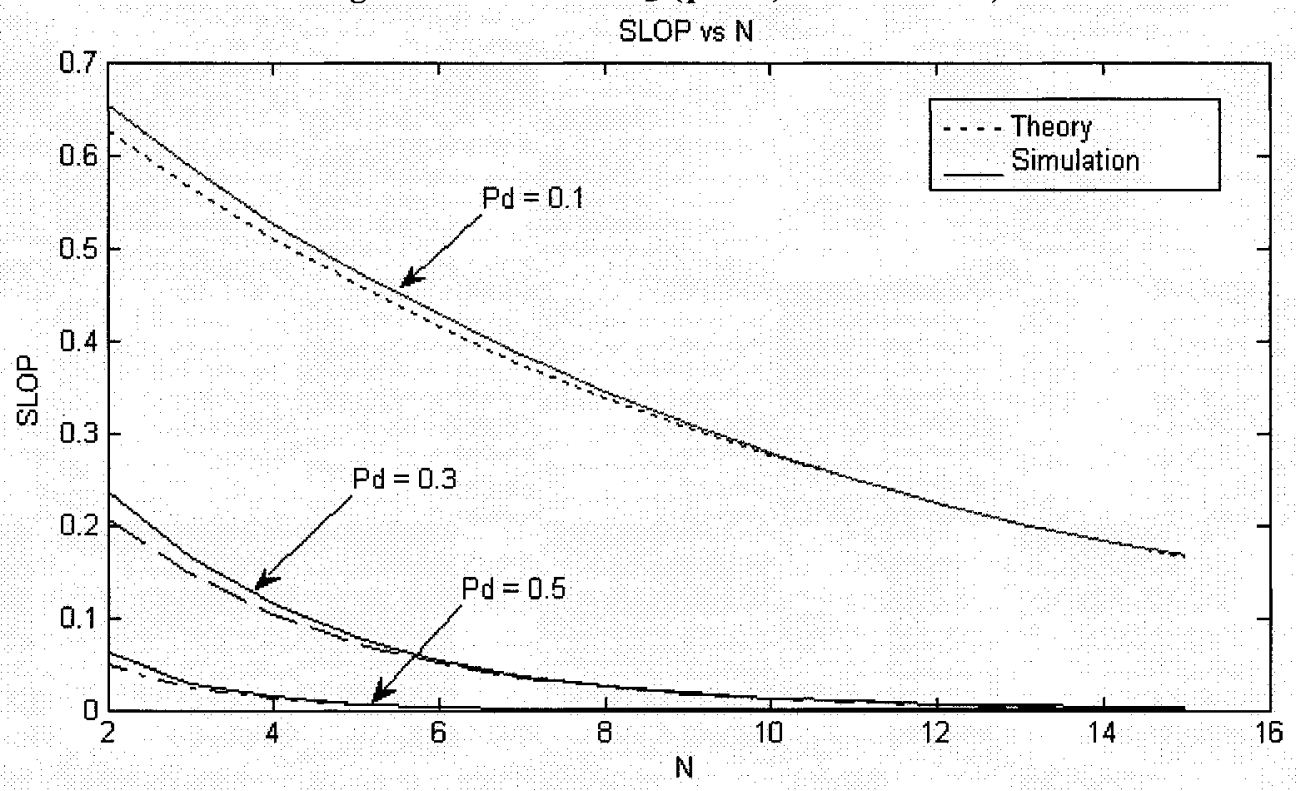

Figure 17: $S L O P$ vs. $N(q=40, H R T=100 \mathrm{~ms})$

The simulation results presented in Figure 16, Figure 17, as well as the ones presented later in chapter 5 , verify the derivations made in section 3.2 and validate modeling the 
acquisition time as an exponential random variable. Further validation of the model can be made by running a simulation experiment to collect a large number of acquisition time samples and plotting their probability density function (pdf). This type of validation is left for future work.

The next set of simulations present the effect of different design variables on the performance of a frequency hopping radio control system. Many design variables exist, of those I mention:

- q: The number of channels used for hopping

- J: The number of blockers (co-channel interferers) present in the band

- $\mathbf{N}$ : The number of states in an $\mathrm{N}$-state lock detector.

The performance metric considered here is the average inter-arrival time of correctly received packets, which represents the average system latency (response).

The first simulation studies the effect of blocking. The Figure below shows the results. The $y$-axis represents the normalized inter-arrival time, while the $\mathrm{x}$-axis represents the partial band blocking ratio. This is the ratio of the number of blocked channels to the total number of channels. Simulation is carried out with a uniform serial-search acquisition engine and a 10-State lock detector. 


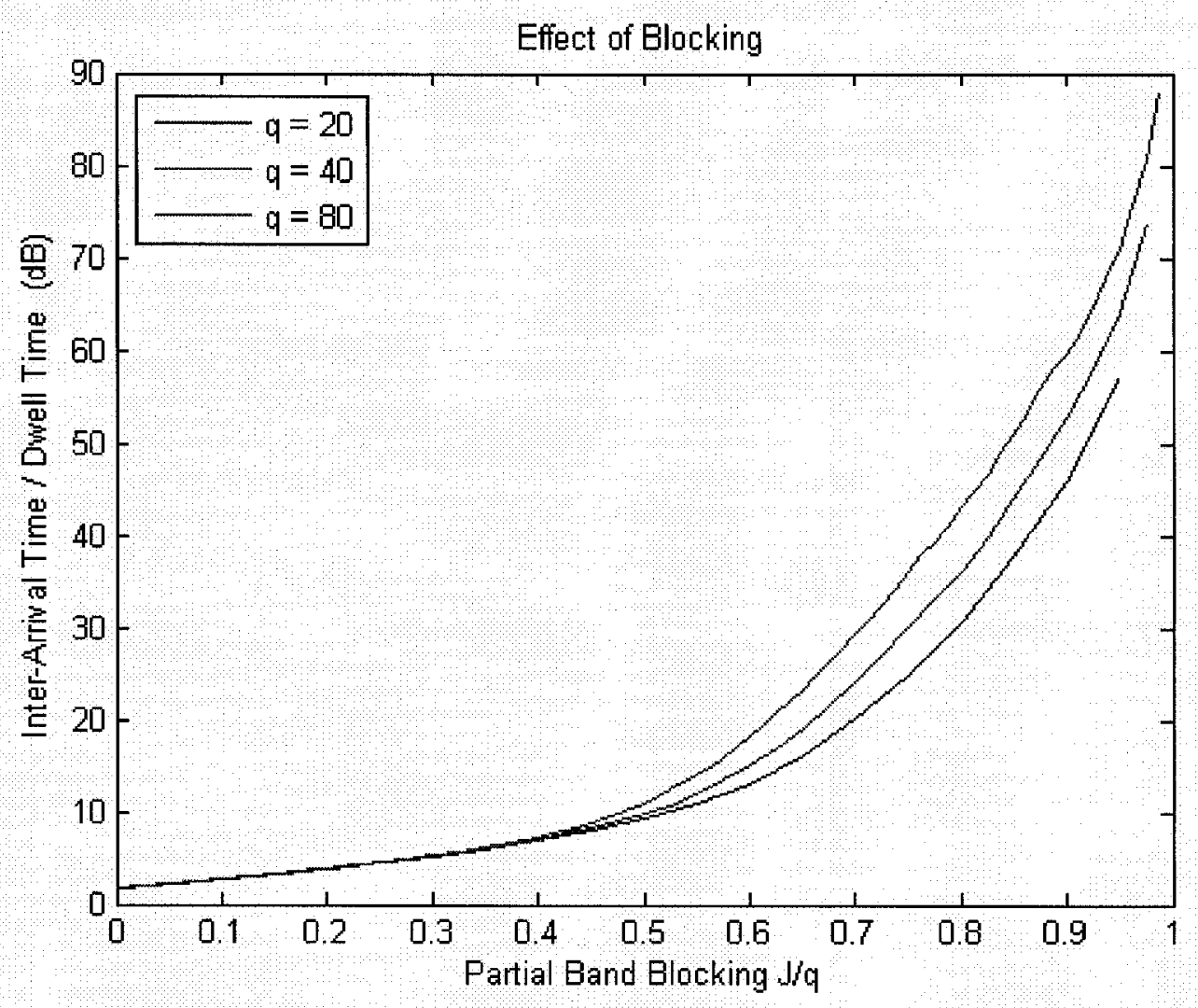

Figure 18: Effect of blocking. $\mathrm{N}=10$

Results show that performance begins to dramatically degrade when $40 \%$ or more of the band is being blocked, regardless of the number of channels being used in hopping. This is expected since a high partial blocking ratio causes the system to frequently loose lock and enter acquisition mode. Acquisition is worse for higher number of channels, therefore performance degradation is worse for 80 channels than it is for 40 and 20 .

The second simulation studies the effect of N-State lock detection on performance. The Figure below shows the results. The y-axis represents the normalized interarrival time, while the $\mathrm{x}$-axis represents the number of states in an N-State lock detector. The 
simulation is carried out at a high partial band blocking ratio $(\mathrm{J} / \mathrm{q}=75 \%)$. Uniform serial search acquisition is used.

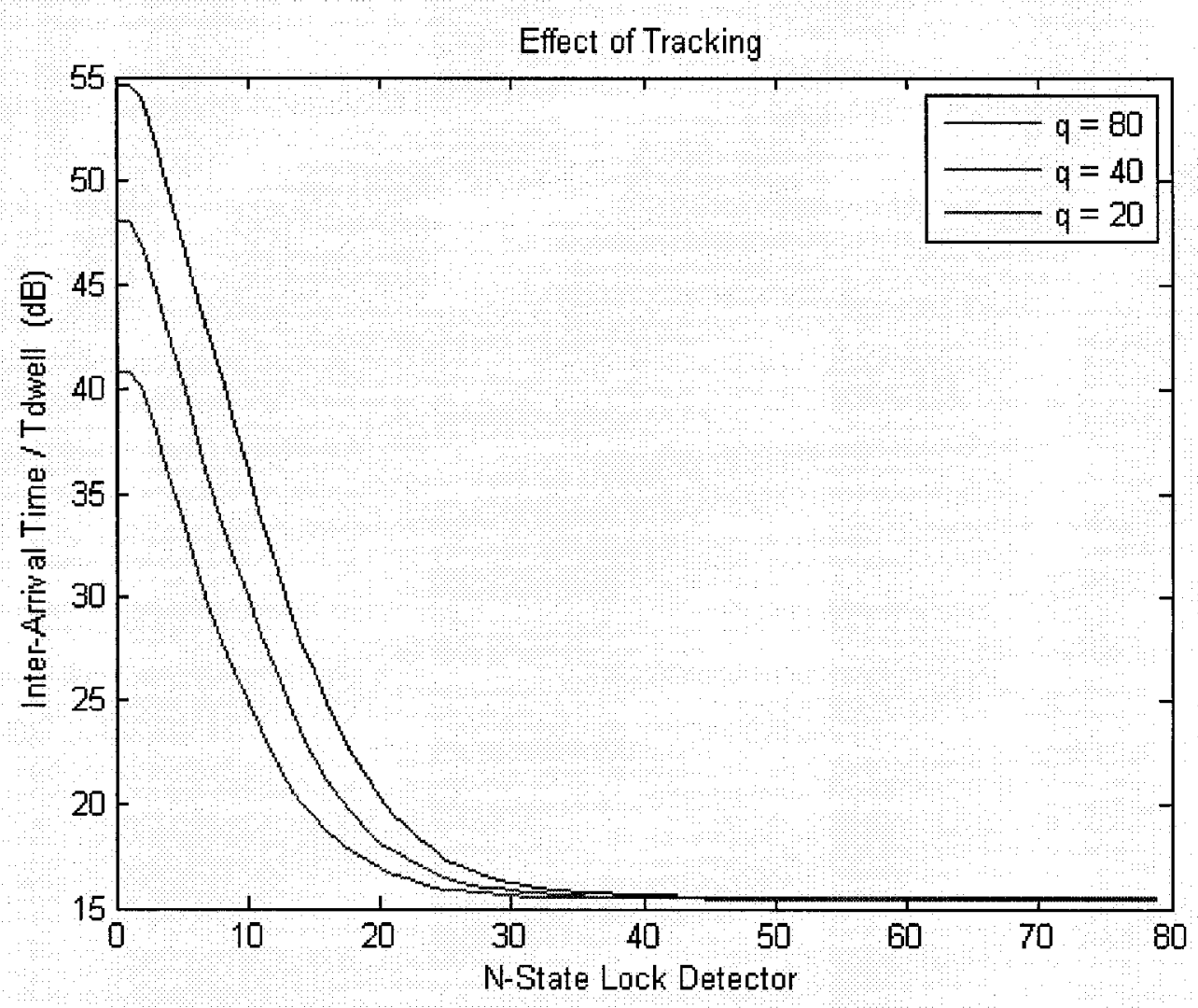

Figure 19: Effect of Tracking. J/q $=\mathbf{0 . 7 5}$

Results show the importance of maximizing the number of states in the N-state lock detector. As $N$ increases, performance improves, to the point where performance of systems operating over different number of channels converges at approximately $N>40$. This is expected since higher $N$ leads to a lower number of acquisition attempts and therefore smaller average inter-arrival time.

The third simulation studies the effect of varying the number of hopping channels. The Figure below shows the results. The y-axis represents the normalized inter-arrival time, 
while the $\mathrm{x}$-axis represents the number of channels used in hopping. The simulation is carried out with uniform serial search acquisition engine and a 10-state lock detector.

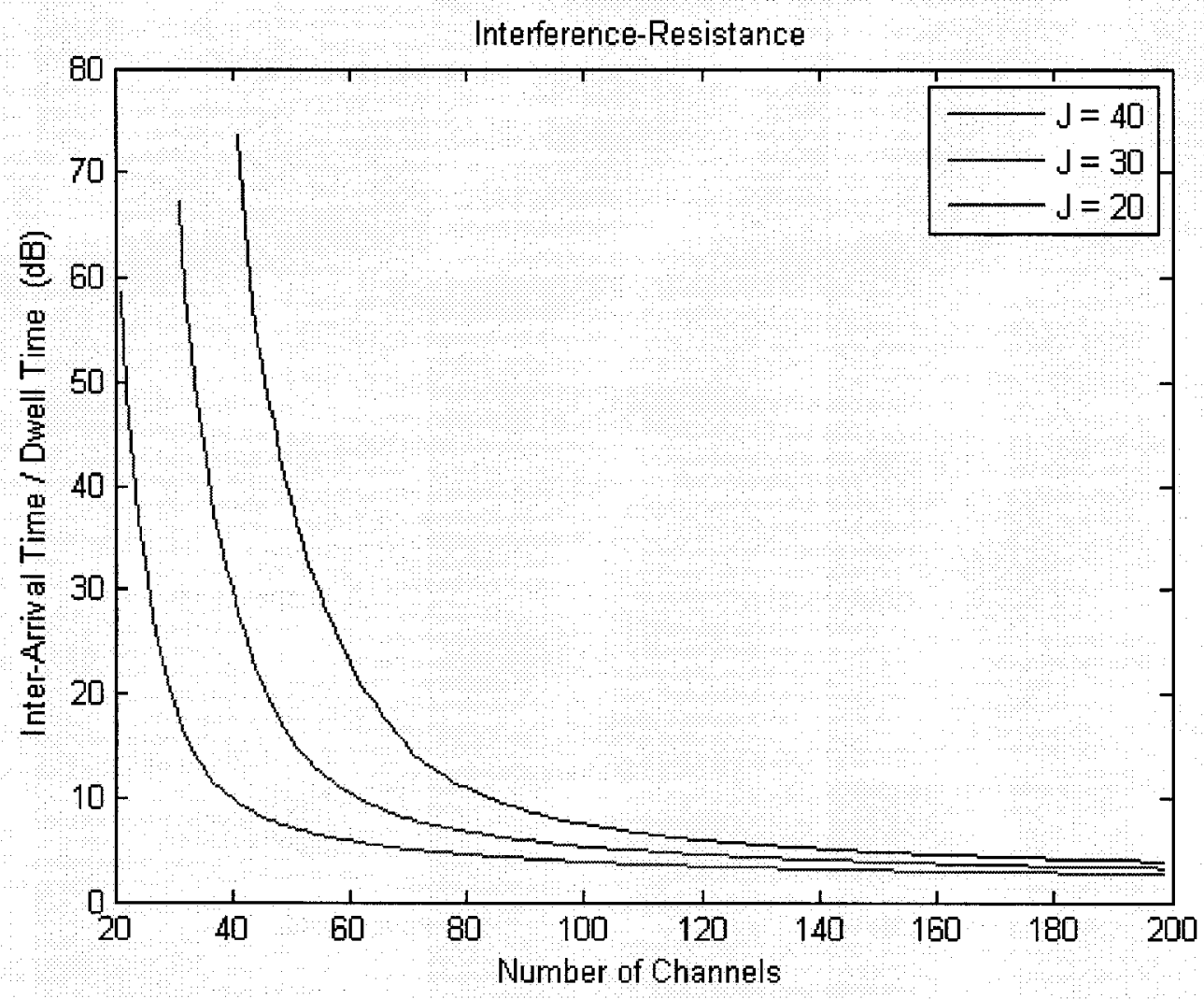

Figure 20: Interference resistance due to increasing number of channels, $N=10$

Results show that increasing the number of channels can be used to effectively combat interference. For a given number of blocked channels, performance improves as the number of channels increase. This happens because the average PER of the system decreases as $q$ increases.

Another way to explain the results in the Figure 20 is in terms of acquisition and tracking. As the number of free channels in the system increases, the chances of finding a free channel for proper communication increases and therefore the system remains in tracking 
mode longer. The number of acquisition attempts therefore decrease and the overall performance of the system improves. 


\section{Implementation}

This section presents the design and implementation of a packet-based frequency hopping system. The system operates in the $2.4 \mathrm{GHz}$ ISM band and uses readily-available low cost Commercial-Off-The-Shelf hardware.

This section has four components. Firstly I present the hardware design. Secondly I present the firmware design. Thirdly I present the experimental procedure used for evaluating the performance of the system. The performance metric considered here is $S L O P$. And finally I present the experimental results. The purpose of the experimental results is to validate the theoretical derivations made in chapter 3 and the simulation results presented in chapter 4 .

\subsection{Hardware Design}

A radio control unit consists of two main components: a microcontroller and a wireless transceiver. The microcontroller used here is the CY8C29466 from Cypress Semiconductor [19], more commonly referred to as Programmable System-on-Chip (PSoC). The wireless transceiver used here is the nRF24L01 from Nordic Semiconductor [38]. First I present an overview on PSoC technology, followed by an overview on the nRF24L01. 


\subsubsection{Programmable System-on-Chip}

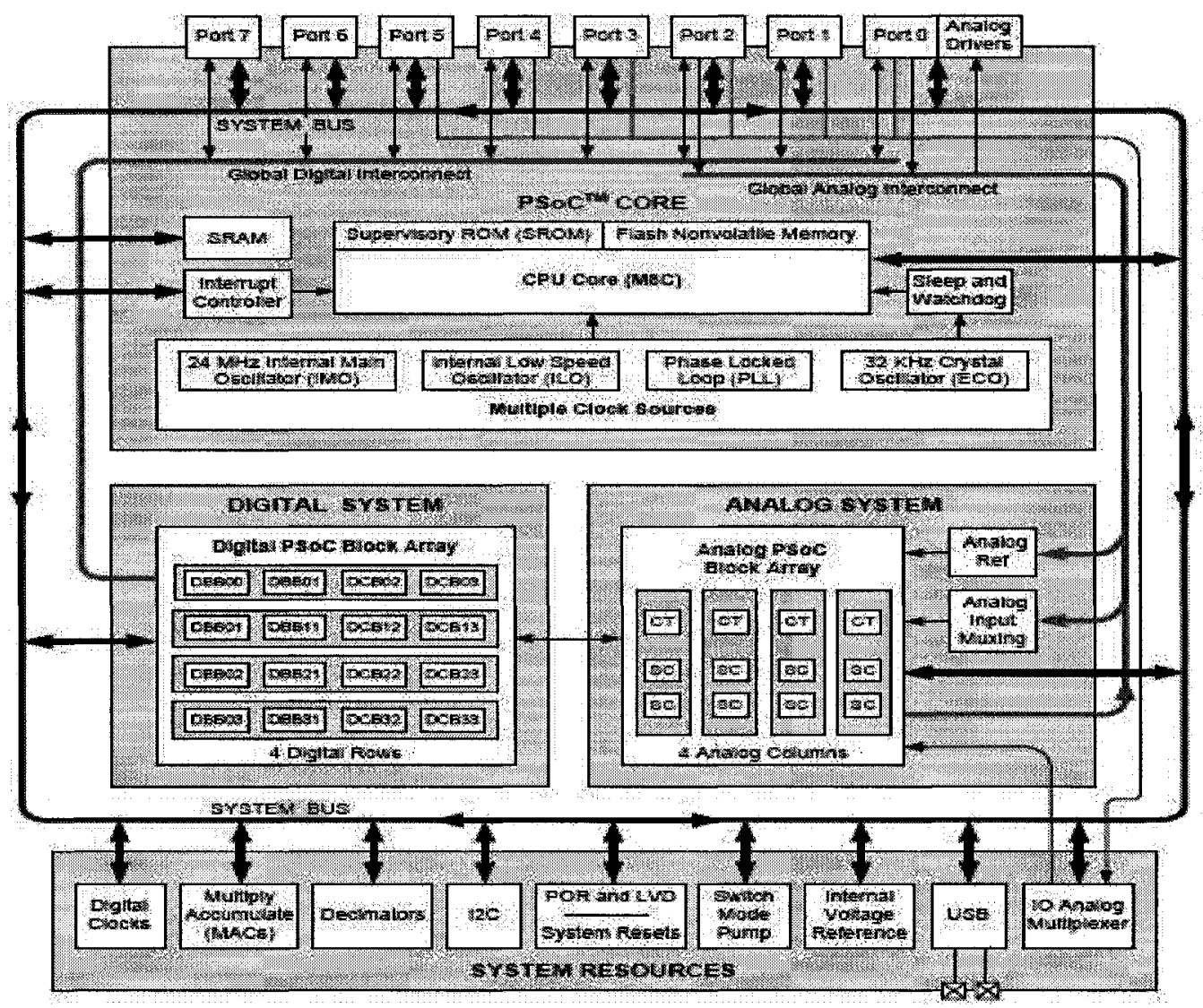

Figure 21: PSoC block diagram

A PSoC consists of four main elements[19], as shown above in the Figure 21:

1- The PSoC core is common to all PSoC families. It contains a proprietary $24 \mathrm{MHz}$ M8C CPU core, on-chip RAM and flash memories, multiple clock sources, a sleep-and-watchdog timer, and an interrupt controller.

2- The Digital System contains a number of Digital Blocks. Each Digital Block can be configured (and reconfigured) to perform one of several digital functions such as 32-bit timer, 16-bit PWM, UART and SPI.

3- The Analog System contains a number of analog blocks. Each analog block can be configured and reconfigured to perform one of several analog functions such as 
filters, amplifiers, tone generators, ADC's, and DAC's.

4- Finally, System Resources provide additional PSoC capability. They include multiply-accumulates (MAC), decimators, I2C, a Switch Mode Pump (SMP), and a full speed (12Mbps) USB.

All of the elements mentioned above are connected through a configurable digital and analog interconnect switching fabric. This fabric enables different blocks to be connected to each other, and to drive or be driven from any of the $\mathrm{I} / \mathrm{O}$ pins available on-chip. The Analog System, the Digital System, and the interconnect form the configurable core of the PSoC device.

In this thesis, I am concerned primarily with the configurable digital system of the PSoC. For more information on the rest of the system, including the PSoC core, the Analog System, and the System Resources please refer to [19] and [18].

The configurable digital system consists of three main components, as shown in Figure 22 below:

1. The Global Digital Interconnect (GDI)

2. The Row Digital Interconnect (RDI)

3. The Digital Blocks (DB) 


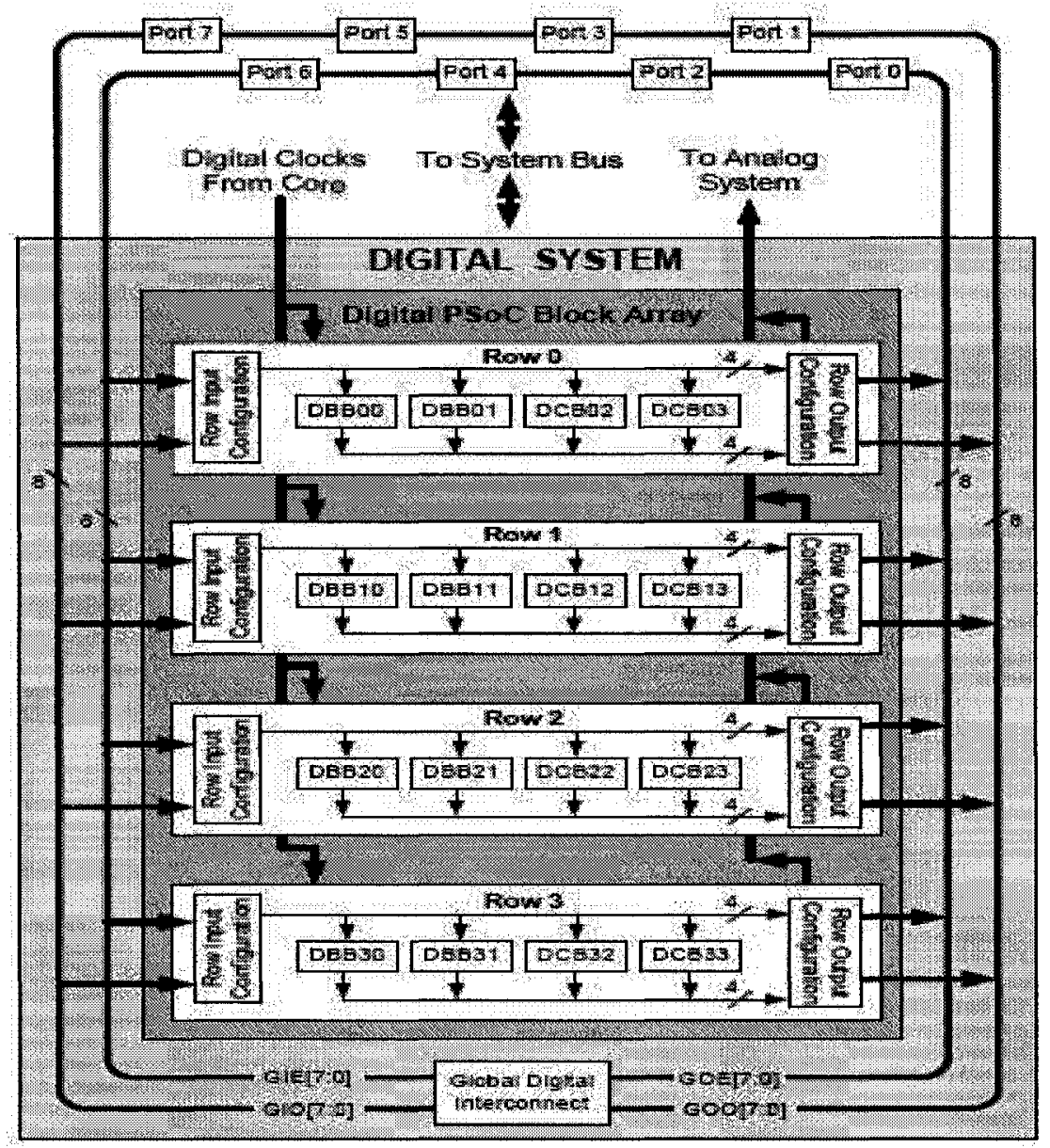

Figure 22: PSoC digital system

The Digital Blocks can be configured to perform one of seven peripheral functions: Timer, Counter, Pulse-Width Modulator (PWM), Pseudo-Random Sequence (PRS), Cyclic Redundancy Check (CRC), Serial Peripheral Interface (SPI), and a full duplex UART. Each Digital Block provides 8 bits of resolution (8-bit timer, 8-bit counter ...etc.). Multiple Digital Block can be chained together to provide higher resolution functions.

The RDI and GDI form the interconnect that allows signal routing to and from the Digital Blocks. The RDI connects the outputs and inputs of Digital Blocks to the GDI. The GDI in turn connects the RDI to the on-chip I/O pins. Together, the RDI and GDI can route 
the inputs and outputs of any of the Digital Blocks between each other and to any of the on-chip I/O pins.

Figure 23 below shows a typical PSoC application that uses a Pulse-Width-Modulated (PWM) Output. Digital Block 0 is configured as an 8-bit PWM whose output is routed to pin 0 of port 0 through the configurable digital interconnect.

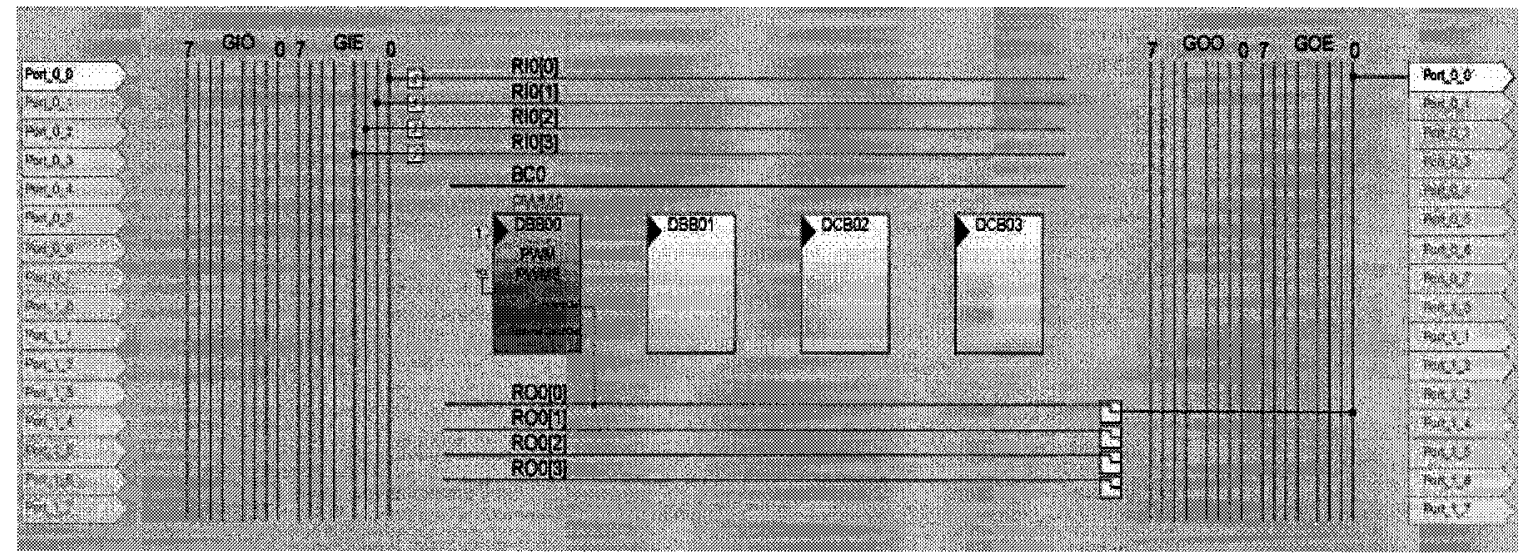

Figure 23: DB00 is configured as a PWM whose output is routed to pin 0 of port 0

Configuration of the Digital Blocks and the digital interconnect is done by programming various RAM-based registers. Specific configurations of these registers can be stored in the on-chip flash and loaded into RAM whenever needed, thus allowing for dynamic reconfiguration of the $\mathrm{PSoC}$.

The GDI consists of four 8-bit buses which allow signals to pass from the core to the pins and vice versa. Two of the buses are input buses called Global Input Odd (GIO) and Global Input Even (GIE). The other two are output buses called Global Output Odd (GOO) and Global Output Even (GOE). Odd busses connect to odd I/O ports such as ports $1,3,5$, and 7 . Similarly, even buses connect to even $\mathrm{I} / \mathrm{O}$ ports such as ports $0,2,4$, 
and 6. The four GDI buses are shown as vertical green in Figure 23. The Figure shows the output of the first Digital Block being routed to output pin 0 of port 0 through the GOE_0.

The RDI consists of four Row Input nets (RI[3:0]) and four Row Output nets (RO[3:0]). The input nets can be seen in Figure 23 as the four red horizontal lines. Their job is to route signals from the GDI to the inputs of the Digital Blocks. Each Row Input contains a 4-to-1 multiplexer whose inputs are fed by four GDI lines. The outputs of these muxes are Row Inputs (RI[x]). This means that any single Row Input net can connect to one of four possible GDI lines, as can be seen to the left of Figure 24 below.

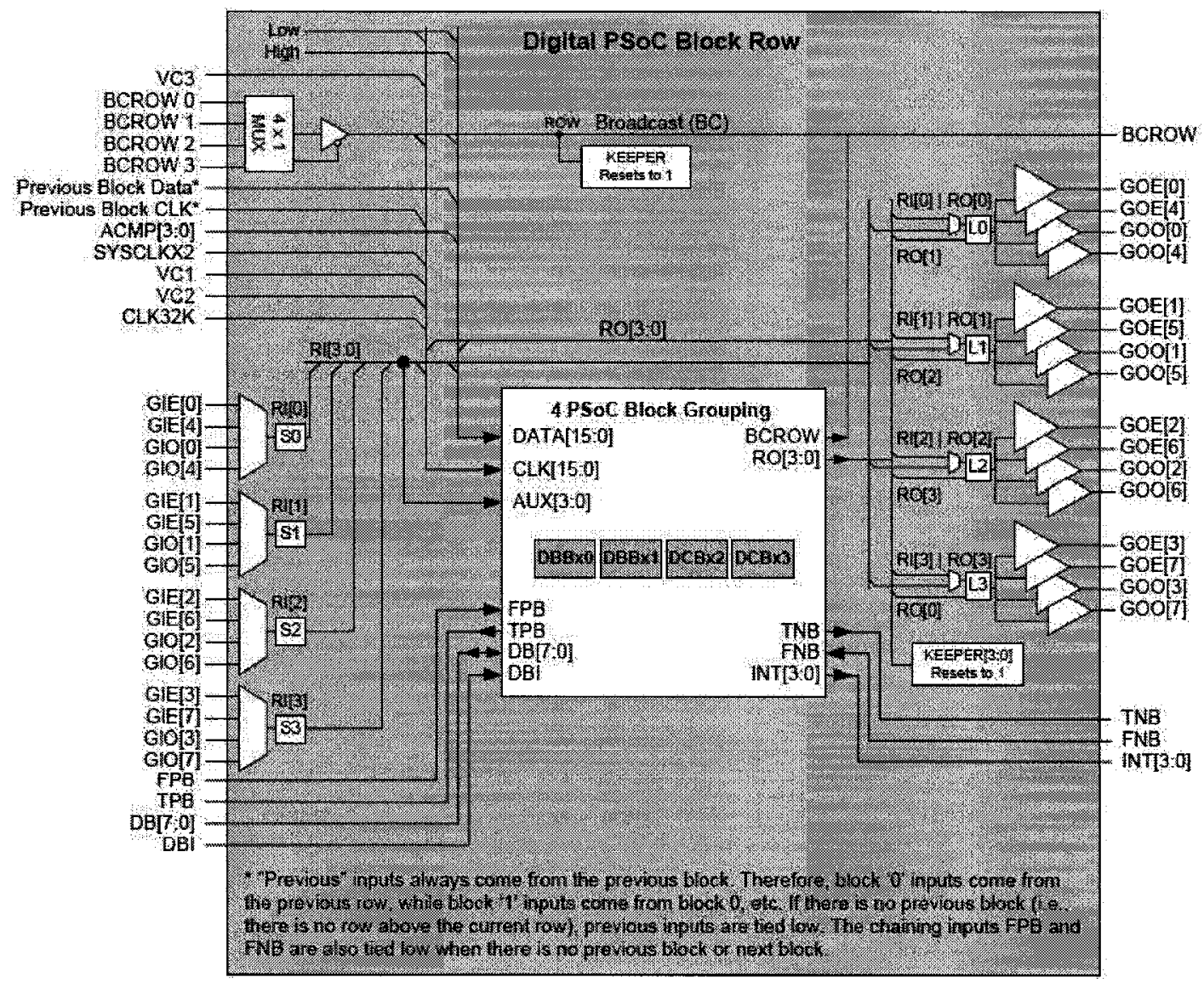

Figure 24: Digital PSoC Block Row Structure 
Each of the four muxes in a Row Input can be controlled through the Row Input Register. As with all other configuration registers, the Row Input Register is RAM-based. This means that it can be configured during start up, as well as during normal operation of the PSoC. This allows for dynamic reconfiguration of the input nets of the PSoC. Figure 25 below shows an example where Digital Block 0 is configured as an input capture 8-bit timer whose input is fed from pin 0 of port 0 through Row Input RI[0].

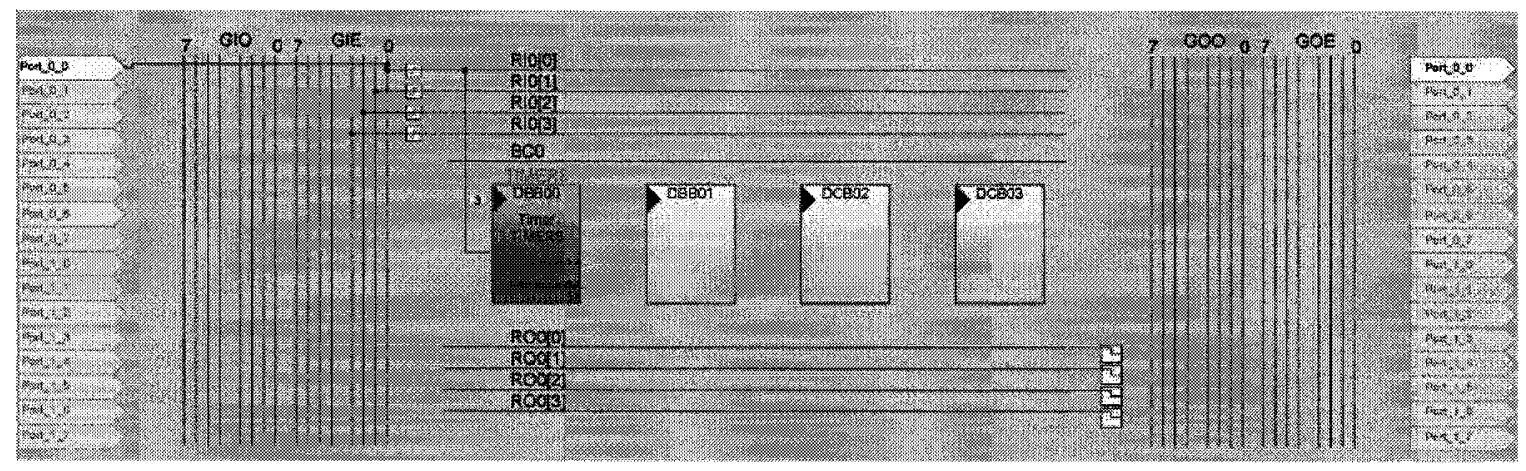

Figure 25: DB00 is configured as an input-capture timer whose input is fed from P0.0

The Row Output nets are a bit more involved than the Row Input nets, as can be seen to the right of Figure 24. In the Figure, the block labeled Lx represents a 2-input look-up table (LUT). The LUT allows the user to specify any one of 16 logic functions that can be applied to the two inputs. A more detailed structure of one Row Output is shown in Figure 26 below. The 16 logic functions are shown in Table 1.

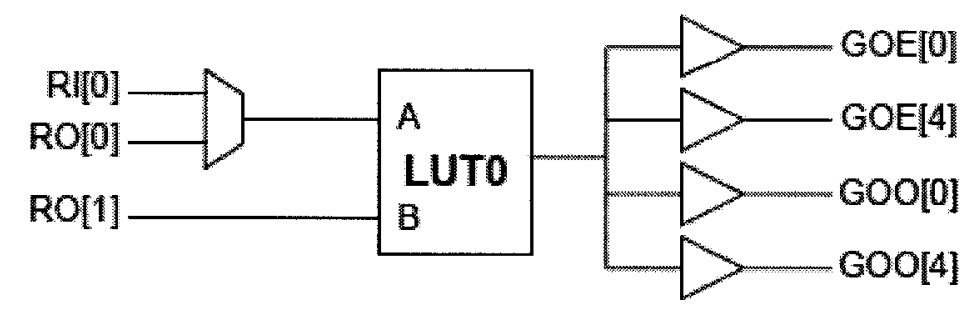

Figure 26: Example of LUT0 configuration 


\begin{tabular}{|c|c|}
\hline LUTx 3.0$]$ & 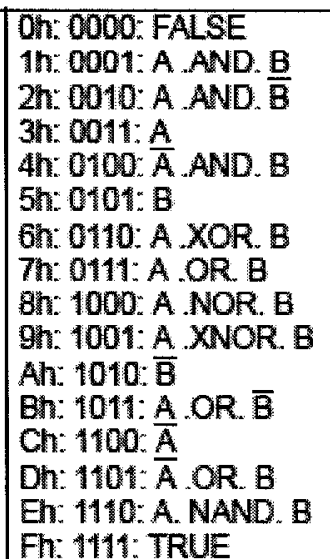 \\
\hline
\end{tabular}

Table 1: LUT logic functions

The output of a LUT can drive as many as 4 GDI lines, through configurable tri-state buffers. The LUT's inputs, outputs, and logic functions are configured through RAMbased registers. This enables dynamic reconfiguration of signal routing from the Digital Blocks to the output pins.

A Digital Block consists of the data path, input multiplexers, output de-multiplexers, configuration registers, and chaining signals, as can be seen in Figure 27 above. Through the RAM-based configuration registers, a Digital Block may be configured to perform any one of seven functions: timer, counter, pulse width modulator (PWM), pseudo random sequence (PRS), cyclic redundancy check (CRC), SPI, and a full duplex UART. Each Digital Block provides 8 bits of resolution (8-bit timer, 8-bit counter ...etc.). Multiple Digital Blocks can be chained together to provide functions with higher resolution. 


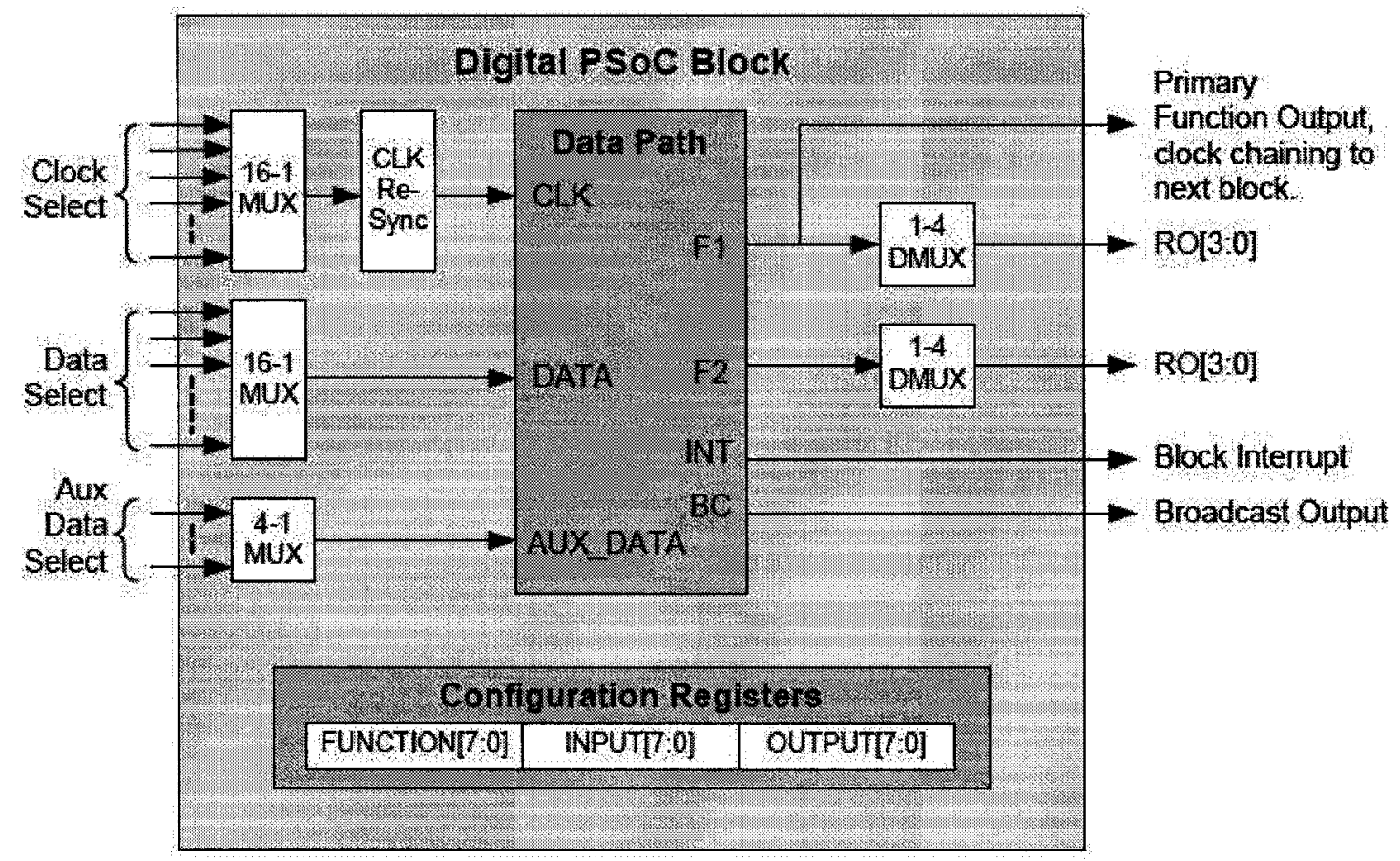

Figure 27: Digital Block Top Level Block Diagram

There are three inputs to a Digital Block: Clock, data, and auxiliary. Typically, each function has a clock and a data input that can be selected from one of 16 possible input sources, through the 16-to-1 input mux. This leaves the auxiliary input, which is selected through the 4-to-1 mux. The auxiliary input is designed specifically to act as the SPI Slave-Select signal for a Digital Block configured in Slave SPI mode.

There are four main outputs to a Digital Block: A primary output, an auxiliary output, a chaining signal, and a block interrupt. The primary and auxiliary outputs can be configured to drive the RDI Row Output bus through two 1-to-4 de-muxes. The chaining signals propagate information from one Digital Block to the next, thus allowing functions with higher resolutions to be implemented. The block interrupt is function-dependant and is used to trigger a vector-based interrupt signal to the PSoC core. 
The input signals, the output signals, and the function of the Digital Block are configured by seven RAM-based registers: Three Data Registers, a Control Register, a Function Register, an Input Register, and an Output Register.

The definition of most of these registers is function-dependant. For example, the definition for each bit in the Control Register changes depending on the selected function, as shown in Table 2 below.

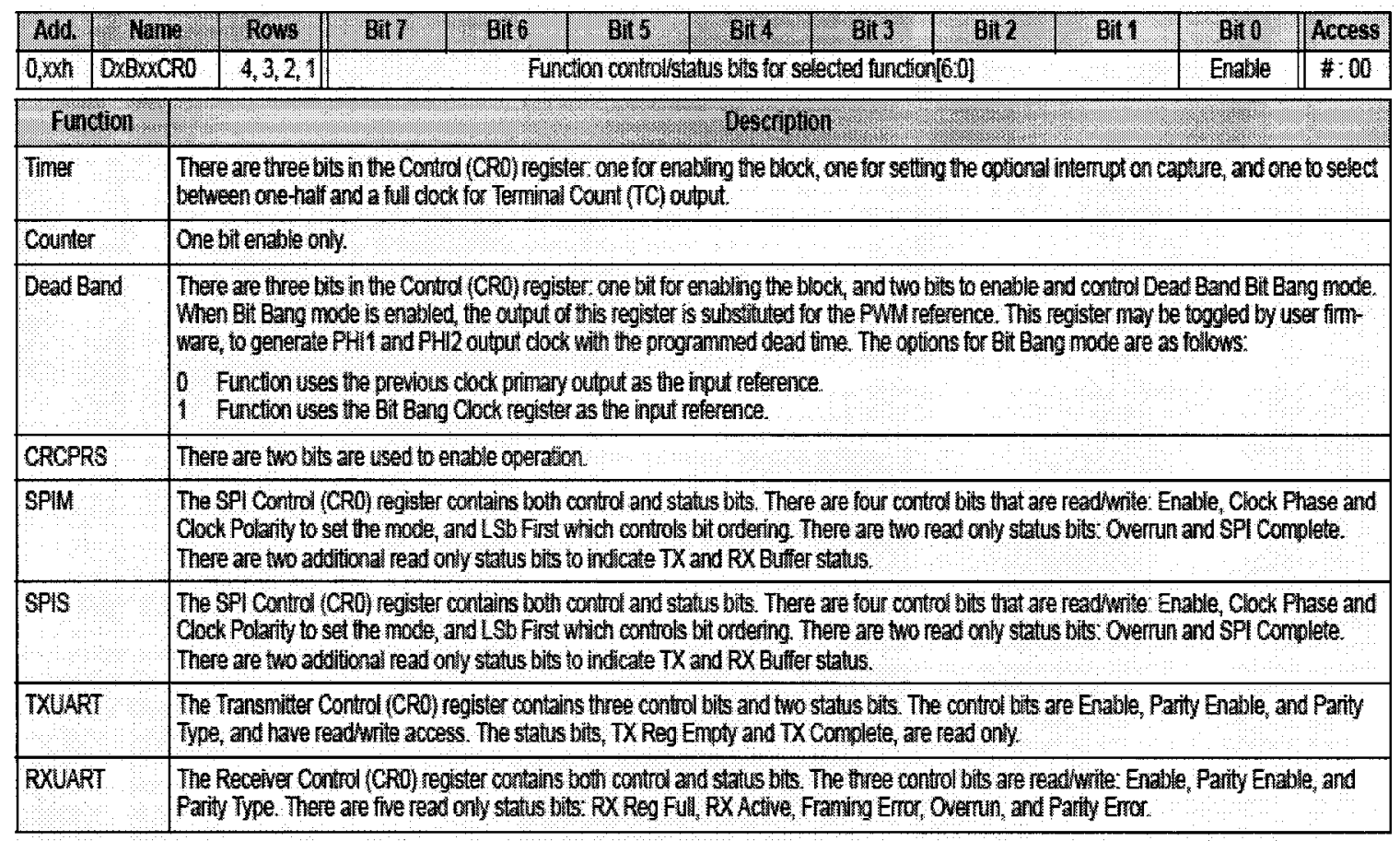

\section{Table 2: Control Register Description}

Similarly, the definition for the three data registers changes depending on the selected function of the Digital Block. For example, when the Timer function is selected, Data Register 0 is automatically configured to hold the Count value, Data Register 1 is automatically configured to hold the Period, and Data Register 2 is automatically 
configured to hold the Capture/Compare value. On the other hand, if the SPI Master function is selected, then Data Register 0 is automatically configured to function as the serial shifter, Data Register 1 is automatically configured to function as the transmit buffer, and Data Register 2 is automatically configured to function as the receive buffer.

The Function register enables selection of the intended function of the Digital Block. The Input Register enables configuration of the three input signals of the Digital Block. The Output Register enables the configuration of the output signals of the Digital Block.

All seven registers of a Digital Block are RAM-based. This enables complete dynamic reconfiguration of any of the Digital Blocks in the PSoC.

\section{Designing with PSoC Designer}

PSoC Designer is an Integrated Development Environment (IDE) [18] used to develop applications for PSoC devices. It contains a Device/Application Editor, a C-Compiler, an Assembler, a Linker, and a Real-Time Debugger.

The Device Editor enables GUI-based configuration of the entire PSoC. It also generates all the necessary function calls required to control the PSoC. Figure $\mathbf{2 8}$ below shows a snapshot of the Device Editor. PSoC global resources can be configured at the top left of the figure. I/O ports can be configured at the lower left of the figure. Digital and Analog Blocks can be selected, placed, and routed in the main area in the middle. 


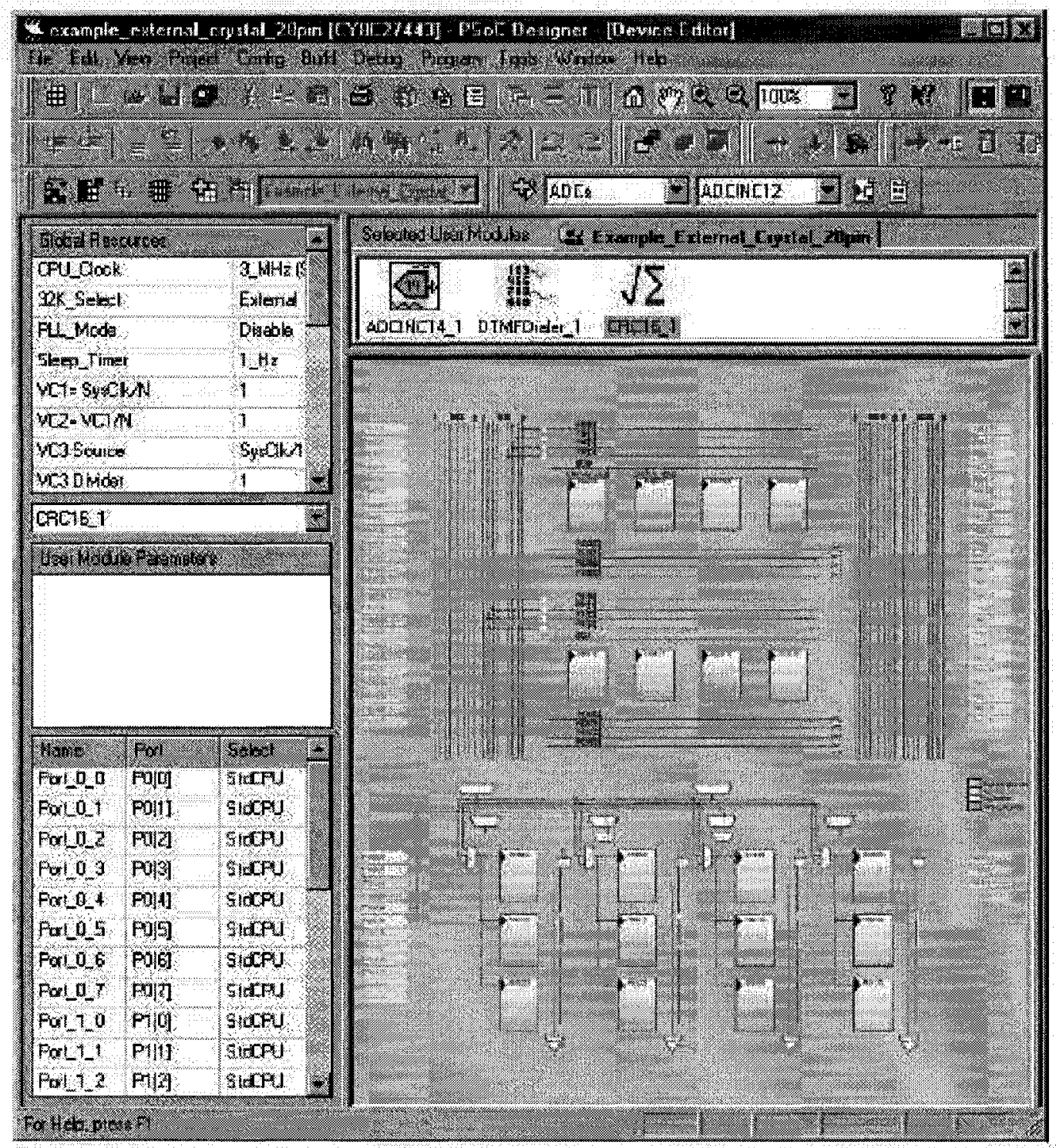

Figure 28: Selected (Yet-to-be-placed) user modules

After configuring, placing and routing a design, the Device Editor will generate application files based on the device configuration. These application files include Application Programming Interface (API) and Interrupt Service Routine (ISR) shells. The Device Editor will also create a datasheet based on the device configuration. 
Once the application files are generated, the Application Editor can be used to create the application code and build it into a HEX file. The HEX file is then loaded into the onchip flash memory of the target device. Flash-programming is performed using the PSoC Programmer tool which accompanies PSoC Designer.

\subsection{2 nRF24L01 2.4GHz Wireless Transceiver}

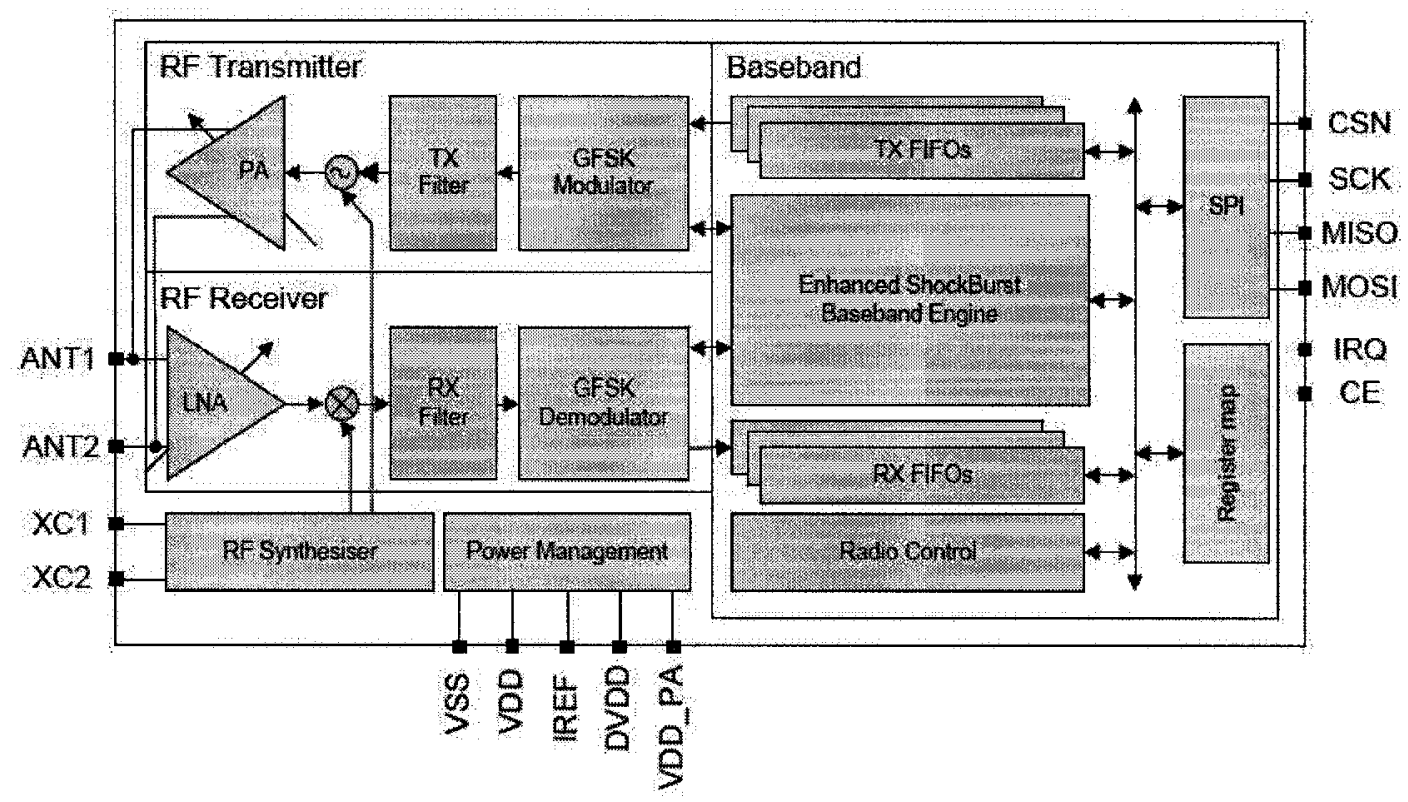

Figure 29: A block diagram of the nRF24L01

The nRF24L01 is a highly integrated $2.4 \mathrm{GHz}$ ISM band transceiver from Nordic Semiconductor [38]. It contains the analog front end and the digital baseband required for bi-directional wireless packet-based communication. The following are some notable features of the nRF24L01:

- Worldwide $2.4 \mathrm{GHz}$ ISM band operation

- Up to $2 \mathrm{Mbps}$ on air data rate

- 11.3mA TX at $0 \mathrm{dBm}$ output power 
- 12.3mA RX at $2 \mathrm{Mbps}$ air data rate

- $900 \mathrm{nA}$ in power down

- $22 \mu \mathrm{A}$ in standby

- On chip voltage regulator

- 1.9 to $3.6 \mathrm{~V}$ supply range

- Automatic packet handling

- Automatic packet transaction handling (ARQ)

- Low cost BOM

$\bullet \pm 60 \mathrm{ppm} 16 \mathrm{MHz}$ crystal

- 5V tolerant inputs

- Compact 20-pin 4x4mm QFN package

The analog front end consists of: a Gaussian Frequency Shift Keying (GFSK) modem, a fully-integrated programmable frequency synthesizer, up and down-conversion mixers, programmable power and low-noise amplifiers, and power management components.

The baseband backend consists of: an advanced packet handler, transmit and receive FIFO buffers, a bank of programmable control and status registers, and a SerialPeripheral Interface (SPI) unit for communication with an external control unit.

At $2 \mathrm{Mbps}$, the $20 \mathrm{~dB}$ bandwidth of the nRF24L01 is specified at $2 \mathrm{MHz}$, and the sensitivity is specified at $-82 \mathrm{dBm}$. The frequency synthesizer generates signals ranging from $2.400 \mathrm{GHz}$ to $2.525 \mathrm{GHz}$ programmable in $1 \mathrm{MHz}$ step resolution. The frequency 
Synthesizer features a Phase-Locked Loop (PLL) capable of settling in 130us over the entire range of frequencies.

The nRF24L01 can be configured in four main modes of operation. It has a built-in state machine that controls the transitions between the different operating modes. The state machine takes input from user defined register values and internal signals. The four modes of operation are: Power-Down (PD), Standby (STDBY), Transmit (TX), and Receive (RX). The figure below defines the transitions between these four modes.

In power down mode the nRF24L01 is disabled with minimal current consumption. Standby mode keeps part of the crystal oscillator active to maintain short start-up times when transitioning to the TX or RX modes. Standby mode is the mode which the nRF24L01 returns to after a packet transaction is complete.

The $\mathrm{RX}$ mode is an active mode where the $\mathrm{nRF} 24 \mathrm{~L} 01$ radio is configured as a receiver. In this mode the receiver demodulates the signals from the RF channel and presents the demodulated data to the on-chip baseband processor. The baseband processor constantly searches for a valid packet. If a valid packet is found (matching address and valid CRC) the payload of the packet is presented in a vacant slot in the RX FIFO. If the RX FIFO is full, the received packet is discarded.

The nRF24L01 remains in RX mode until it is instructed to enter standby or power down modes. If the automatic protocol features (Enhanced ShockBurst ${ }^{\mathrm{TM}}$ ) in the baseband 
protocol engine are enabled, the nRF24L01 enters other modes in order to execute the protocol.

The TX mode is an active mode in which the nRF24L01 transmits a packet. The nRF24L01 stays in TX mode until it finishes transmitting a current packet, after which it returns to standby mode. If the automatic protocol features (Enhanced ShockBurst ${ }^{\mathrm{TM}}$ ) in the on-chip baseband processor are enabled, the nRF24L01 enters other modes in order to execute the protocol.
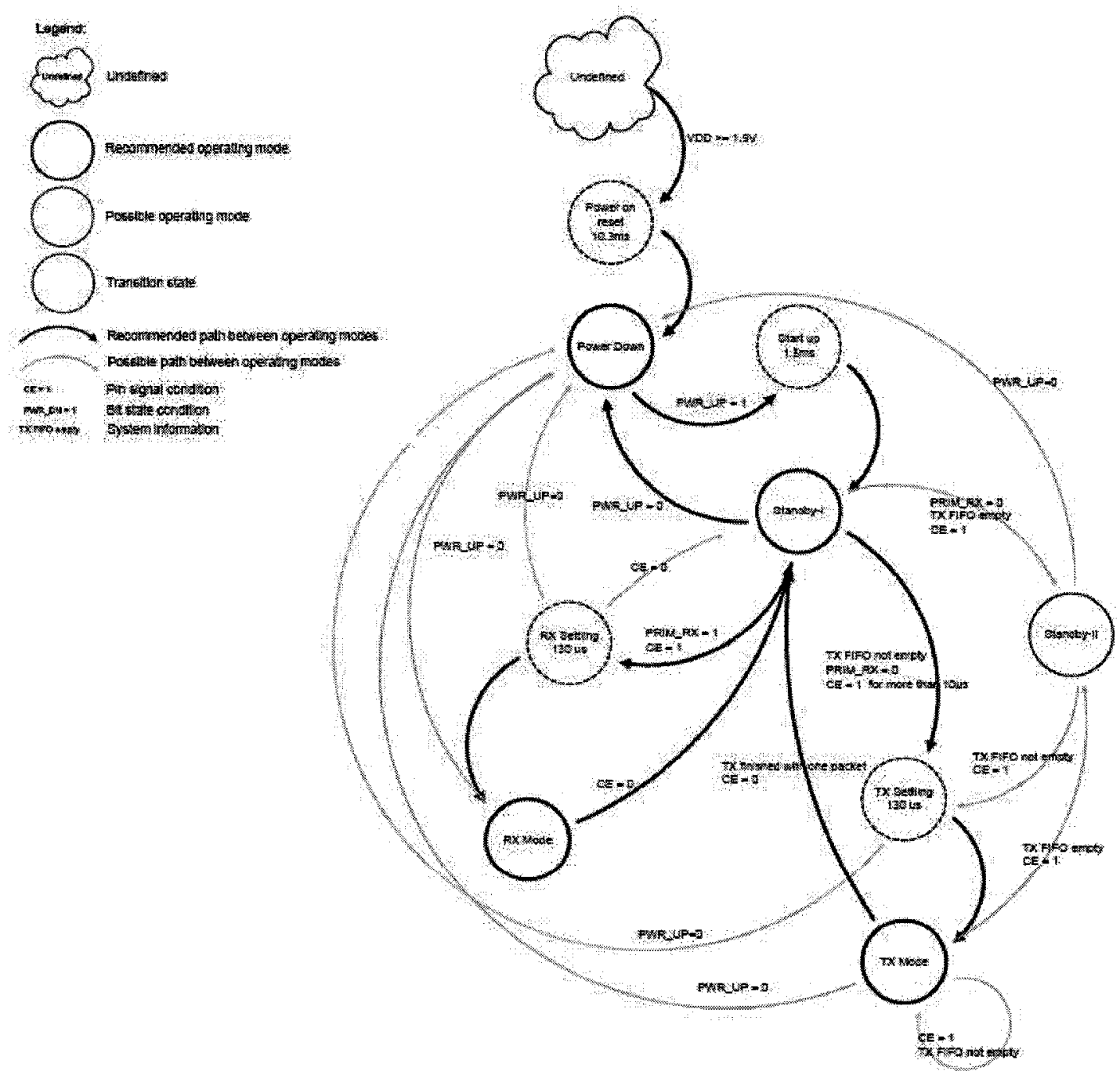

Figure 30: Radio Control Finite State Machine 
Enhanced ShockBurst ${ }^{\mathrm{TM}}$ ) is an on-chip data link layer which features automatic packet assembly, and automatic acknowledgment and re-transmission of packets (Automatic Repeat Request). The packet format supported by Enhanced ShockBurst ${ }^{\mathrm{TM}}$ is shown below.

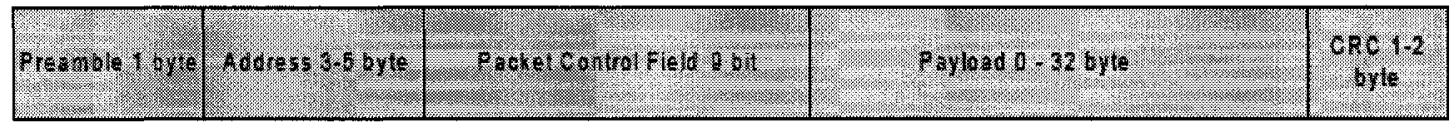

Figure 31: Packet format supported by the nRF24L01

The first field in the packet is the Preamble. The Preamble is a one byte long sequence of alternating bits. The purpose $\mathrm{o}$ the preamble is to aid the demodulator in the detection of binary logic levels.

The Address field contains the address of the Primary Transmitting device (PTX). The length of this address is configurable between 3-5 bytes. At the Primary receiving device (PRX), the address field of a newly received packet is extracted and compared to the programmed PTX address. In the event of a mismatch the packet is discarded.

A 9-bit Packet Control field follows the address field. It contains the Packet Identification (PID) number and the length of the payload (in bytes). The PID is used to distinguish duplicate packets during Automatic-Repeat Request (ARQ) transactions. The payload length is configurable between $0-32$ bytes per packet. 
The last two fields are the payload and CRC fields. The payload filed holds the application data and is configurable in length between $0-32$ bytes. The CRC field is the error detection mechanism in the packet and is calculated over the address, Packet Control, and Payload fields. ARQ transactions are outside the scope of this project and therefore I won't discuss them any further. For more information please refer to the datasheet.

The Data and Control Interface allows access to all of the features of the nRF24L01. It features a digital signal interface, a command interpreter, a configurable register map, and full three-level TX and RX FIFO buffers.

The digital signal interface consists of six digital signals, four of which are used for SPI communications:

- IRQ (this signal is active low and is controlled by three mask-able interrupt sources)

- CE (this signal is active high and is used to activate the chip in RX or TX mode)

- CSN (SPI signal)

- SCK (SPI signal)

- MOSI (SPI signal)

- MISO (SPI signal)

The command interpreter features eleven single-byte SPI commands. The commands are used mainly for reading from and writing to the register map, reading from and writing to the data FIFO buffers, as well as flushing the data FIFO buffers. 
In TX mode, the TX FIFO buffer is used to queue the payload bytes while being transmitted. The IRQ signal is used to assert an active-low interrupt when the transmission of a packet is completed. This interrupt can be used to notify the MCU that the TX FIFO buffer is ready to accept new payload and begin a new transmission.

Similarly during RX mode, the RX FIFO buffer is used to hold the payload extracted from a newly-received packet. The IRQ signal is used to assert an active-low interrupt when the reception of a correct packet is complete. This interrupt can be used to notify the MCU of the newly received payload.

The register map consists of control, status, and data registers. All the registers are accessed using SPI commands. Control (configuration) registers are used to activate or de-activate certain features in the nRF24L01. Status registers are used to convey information regarding the state of the nRF24L01 during a communication session. Finally, the data registers are used to access buffers such as the TX and RX FIFO buffers.

\subsubsection{Development Boards used in Prototyping}

The transmitter and receiver prototypes consist of two main components: the PSoCEval1 Board [56], and the nRF24L01 breakout board. The PSoCEval1 Board is built around the CY8C29466 PSoC and contains most of the peripherals needed to build and test a frequency hopping system. Such peripherals include LED's, switches, LCD module, a regulated $5 \mathrm{~V}$ power supply, a serial port, and a programming interface for downloading firmware into the CY8C29466. 
The board also contains a small breadboard which is ideal for interfacing the CY8C29466 to the nRF24L01 transceiver. The PSoCEval1 Board is shown below in Figure 32 [56]. A schematic of the PSoCEval1 Board is shown in Figure 33 [57].

The nRF24L01 breakout board is used to interface the transceiver to the PSoC. An onboard 3.3V regulator is used to supply appropriate power to the nRF24L01. The breakout board for the nRF24L01 is shown in Figure 34 below [58]. The completed prototype is shown below in Figure 35.

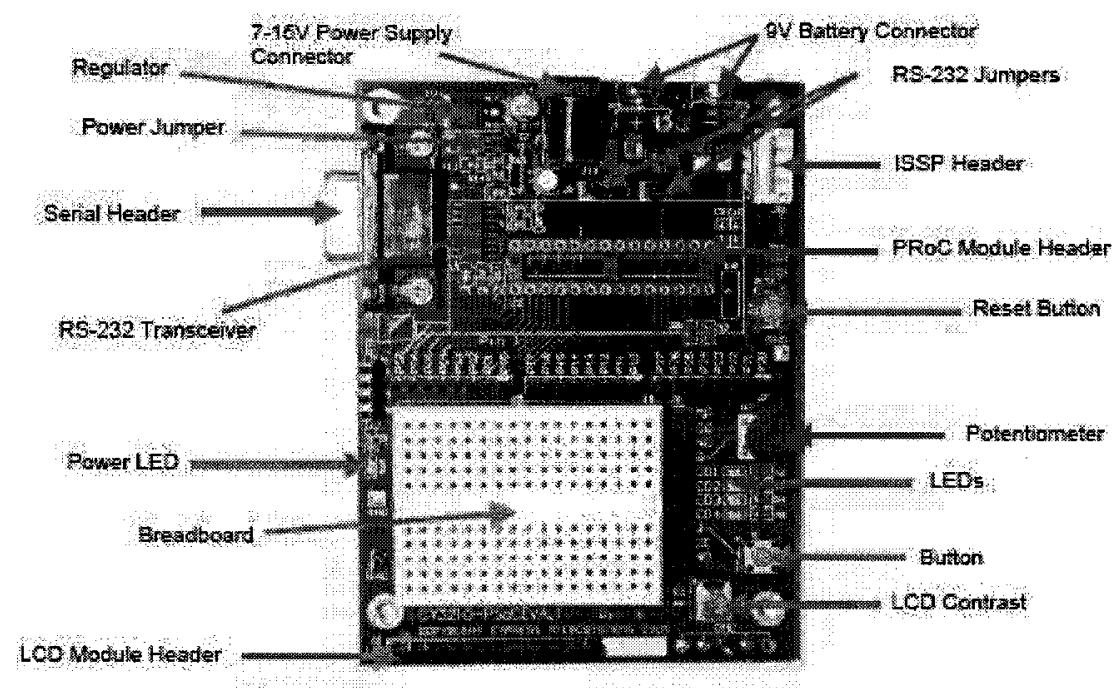

Figure 32: PSoCEval1 Board 


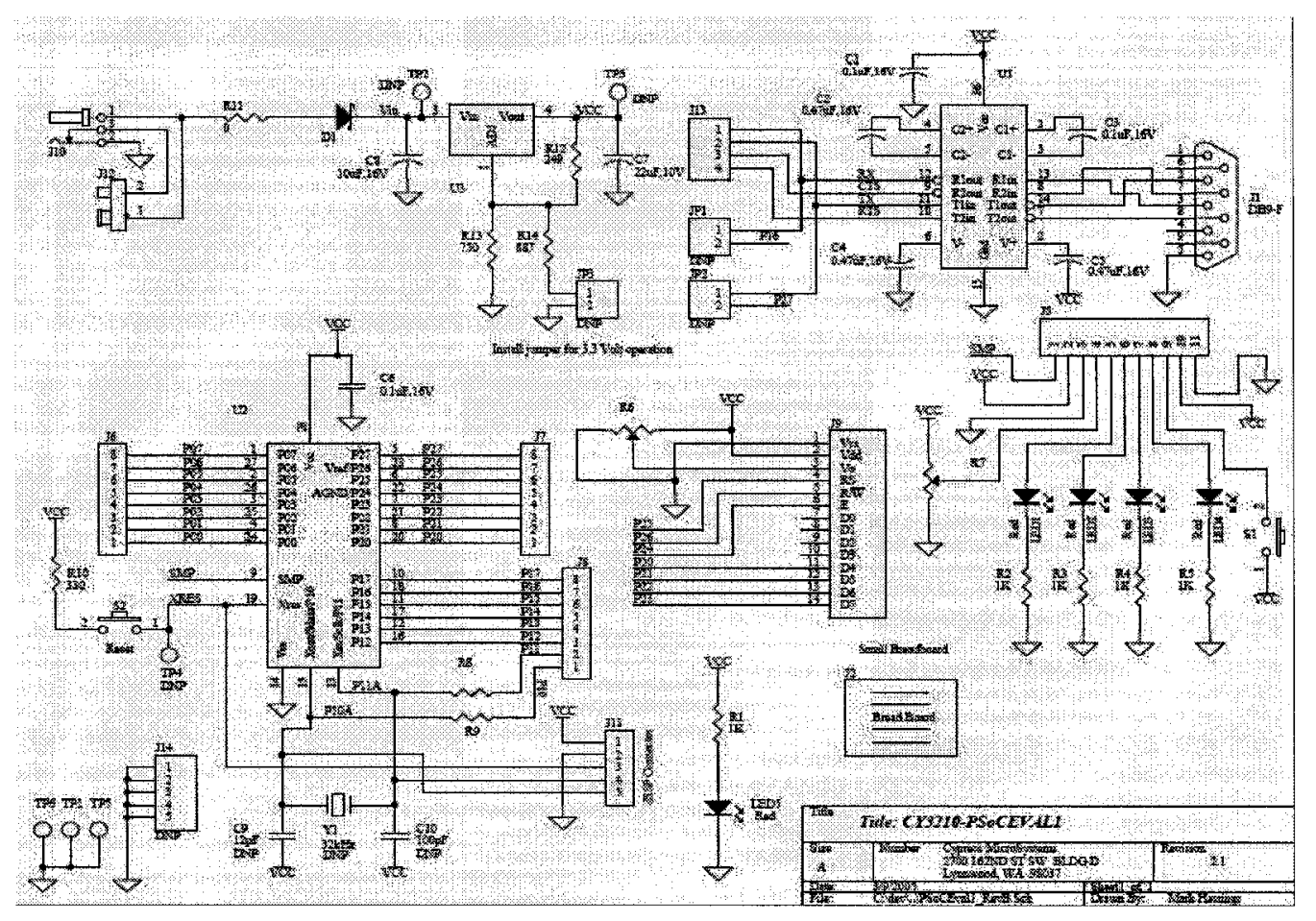

Figure 33: PSoCEval1 Board schematic

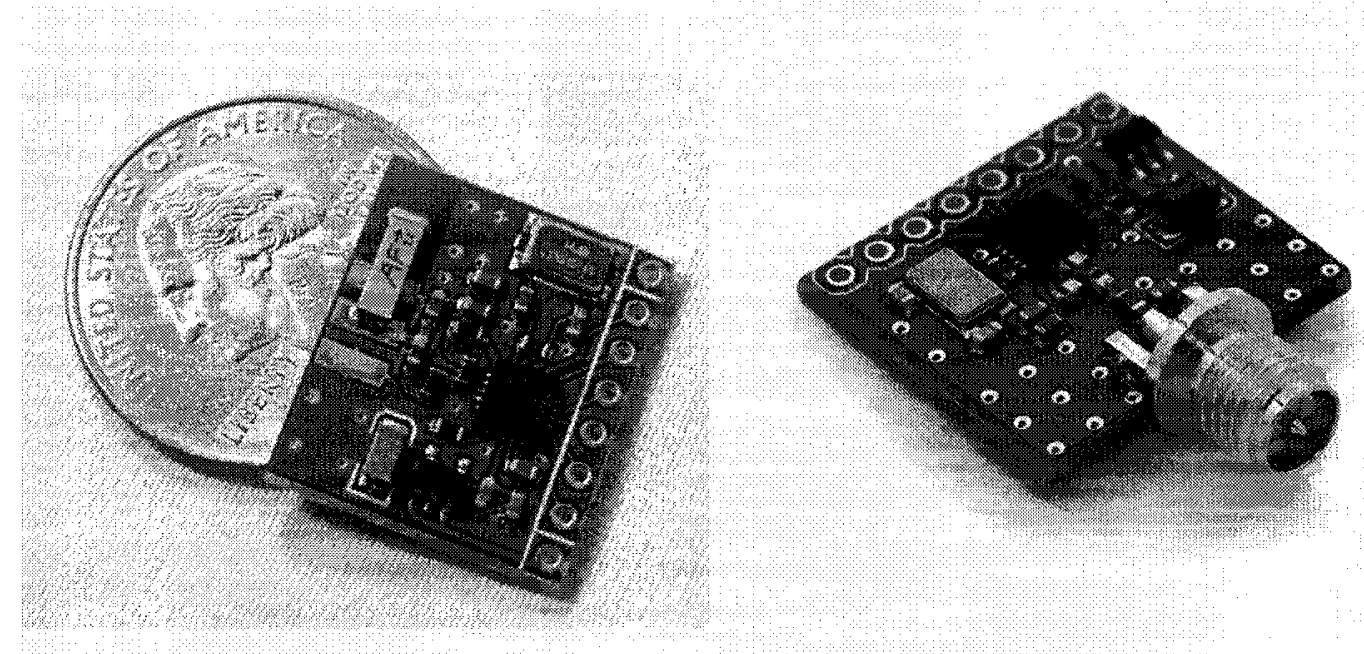

Figure 34: nRF24L01 Breakout Board 


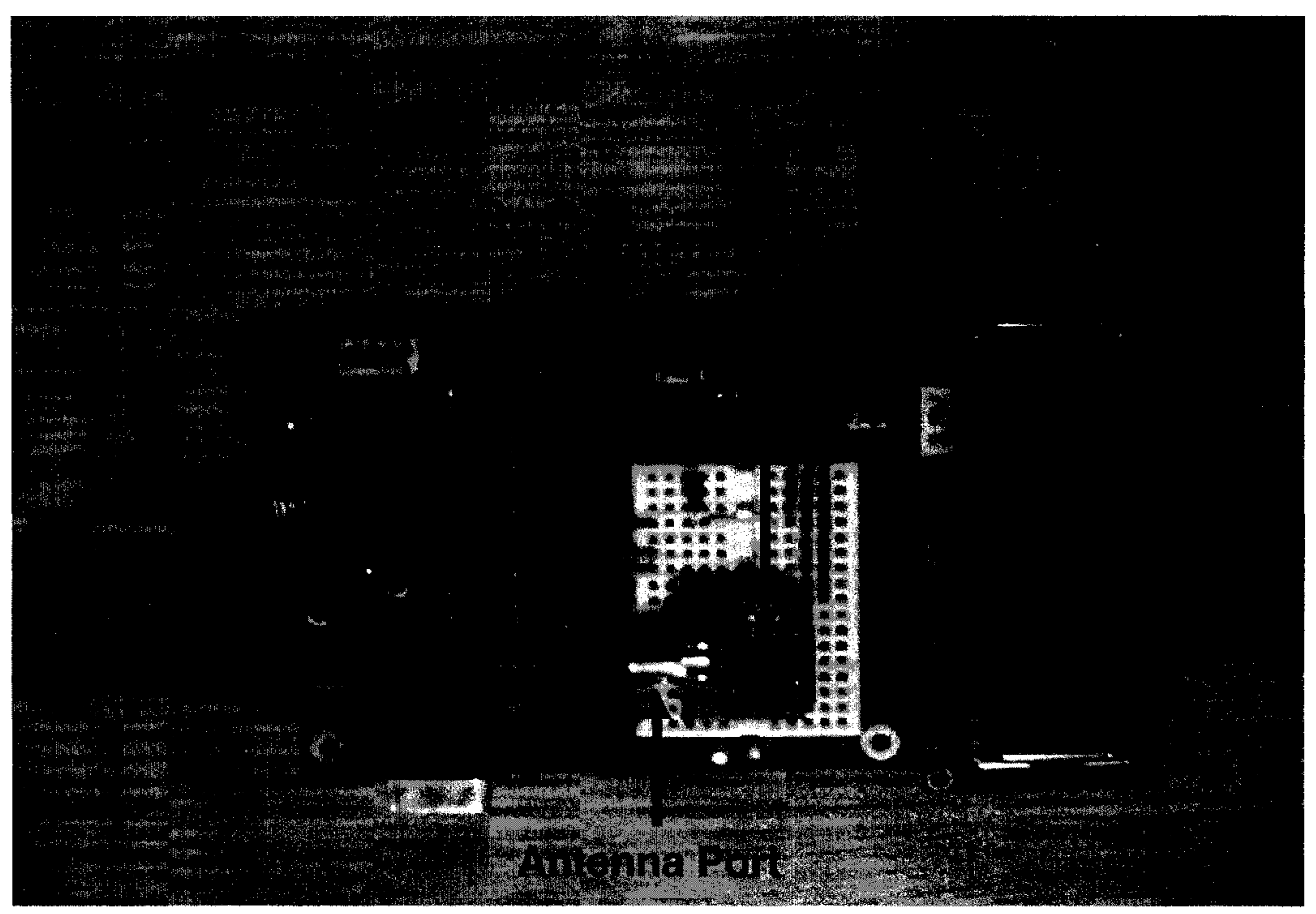

Figure 35: Completed prototype

\subsection{Firmware Design}

The system is designed to replicate simulation settings as much as possible. 40 channels are used for hopping, $N$ is set to 3 , and the dwell time is $1 \mathrm{~ms}$. The transmitter uses a uniformly distributed hopping sequence. The receiver implements uniform serial acquisition with $\mathrm{N}$-state lock detection. First I present the transmitter firmware design, followed by the receiver firmware design.

\subsubsection{Transmitter Firmware}

The transmitter hops over a uniformly distributed pseudorandom sequence of length 40 . An API is developed to control the nRF24L01. The API consists of low-level functions (shown in Table 3 below) and high-level functions (shown in Table 4). 


\begin{tabular}{|l|l|}
\hline Function Prototype & Description \\
\hline Void nRF24L01_setupSPI(void) & Initializes the SPI interface. \\
\hline Void nRF24L01_powerupReset(void) & Resets the nRF24L01 upon power-up. \\
\hline BYTE nRF24L01_readRegister(BYTE address) & $\begin{array}{l}\text { Reads an nRF24L01 register at the } \\
\text { specified address. }\end{array}$ \\
\hline Void nRF24L01_writeRegister(BYTE address, BYTE value) & $\begin{array}{l}\text { Writes value to an nRF24L01 register } \\
\text { at the specified address. }\end{array}$ \\
\hline $\begin{array}{l}\text { void nRF24L01_readBurstRegister(BYTE address, BYTE* data, } \\
\text { BYTE length) }\end{array}$ & $\begin{array}{l}\text { Reads a number of consecutive } \\
\text { nRF24L01 registers starting at the } \\
\text { specified address. }\end{array}$ \\
\hline $\begin{array}{l}\text { void nRF24L01_writeBurstRegister(BYTE address, BYTE* data, } \\
\text { BYTE length) }\end{array}$ & $\begin{array}{l}\text { Writes a number of nRF24L01 } \\
\text { registers starting at the specified } \\
\text { address. }\end{array}$ \\
\hline Void nRF24L01_readRxFIFO(BYTE* payload, BYTE length) & $\begin{array}{l}\text { Reads the specified number of bytes } \\
\text { from the receive FIFO buffer. }\end{array}$ \\
\hline Void nRF24L01_writeTxFIFO(BYTE* payload, BYTE length) & $\begin{array}{l}\text { Writes the specified number of bytes } \\
\text { to the transmit FIFO buffer }\end{array}$ \\
\hline Void nRF24L01_strobe(BYTE command) & $\begin{array}{l}\text { Sends the specified command to the } \\
\text { nRF24L01. }\end{array}$ \\
\hline Void nRF24L01_wait2ms(void) & Provides a blocking 2ms delay. \\
\hline
\end{tabular}

Table 3: Low-level nRF24L01 API calls

\begin{tabular}{|l|l|}
\hline Function Prototype & Description \\
\hline void nRF24L01_start(void) & $\begin{array}{l}\text { Initializes the SPI interface. Powers up } \\
\text { the nRF24L01 and programs its } \\
\text { registers. }\end{array}$ \\
\hline void nRF24L01_setParameter(BYTE address, BYTE parameter) & $\begin{array}{l}\text { Sets the specified parameter in the } \\
\text { specified register. }\end{array}$ \\
\hline $\begin{array}{l}\text { void nRF24L01_clearParameter(BYTE address, BYTE } \\
\text { parameter) }\end{array}$ & $\begin{array}{l}\text { Clears the specified parameter in the } \\
\text { specified register }\end{array}$ \\
\hline Void nRF24L01_setMode(BYTE mode) & $\begin{array}{l}\text { Sets the nRF24L01 in the specified } \\
\text { mode: TX, RX, and STDBY. }\end{array}$ \\
\hline void nRF24L01_setPower(BYTE power) & Programs the output power level. \\
\hline void nRF24L01_setChannel(BYTE rf_channel) & Programs the desired RF channel. \\
\hline
\end{tabular}

Table 4: High-level nRF24L01 API calls

The Main function in the transmitter consists of a simple while loop that instructs the nRF24L01 to hop and transmit 20-Byte long packets. Hopping is periodic at $1 \mathrm{~ms}$ time intervals. The pseudo-code for Main is show in Listing 1 below.

\section{Loop forever \{}

Repeat over the number of packets to be sent \{

Wait for $1 \mathrm{~ms}$ timeout 
Set the new channel in the hopping sequence

Upload the payload in the TX FIFO

Instruct the nRF24L01 to transmit the packet

Increment the number of packets sent

Display the number of packets sent on the LCD

If reached the maximum number of packets to send, quit

\}

\}

$$
\text { If reached the maximum number of packets to send, quit }
$$

Listing 1: Transmitter Main function

The uniformly distributed pseudorandom hopping sequence used in the application is of length 40 and is shown below in Listing 2.

$$
\begin{gathered}
16,37,4,27,35,2,20,36,1,25,10,6,31,14,39,5,28,38,30,12,34,15,29,19,11 \\
32,0,23,33,8,21,18,3,13,7,22,26,9,17,24
\end{gathered}
$$

Listing 2: Uniformly distributed pseudorandom hopping sequence used in the application

\subsubsection{Receiver Firmware}

The receiver is an event-triggered FSM which implements uniform serial acquisition with N-state lock detection. Two events drive the FSM: Timeout event, and Packet-received event. The receiver FSM is shown in Figure 36 below. Please note that the implemented receiver FSM is an exact replica of the one built in the simulator, shown in Figure 15 in the simulation section. 


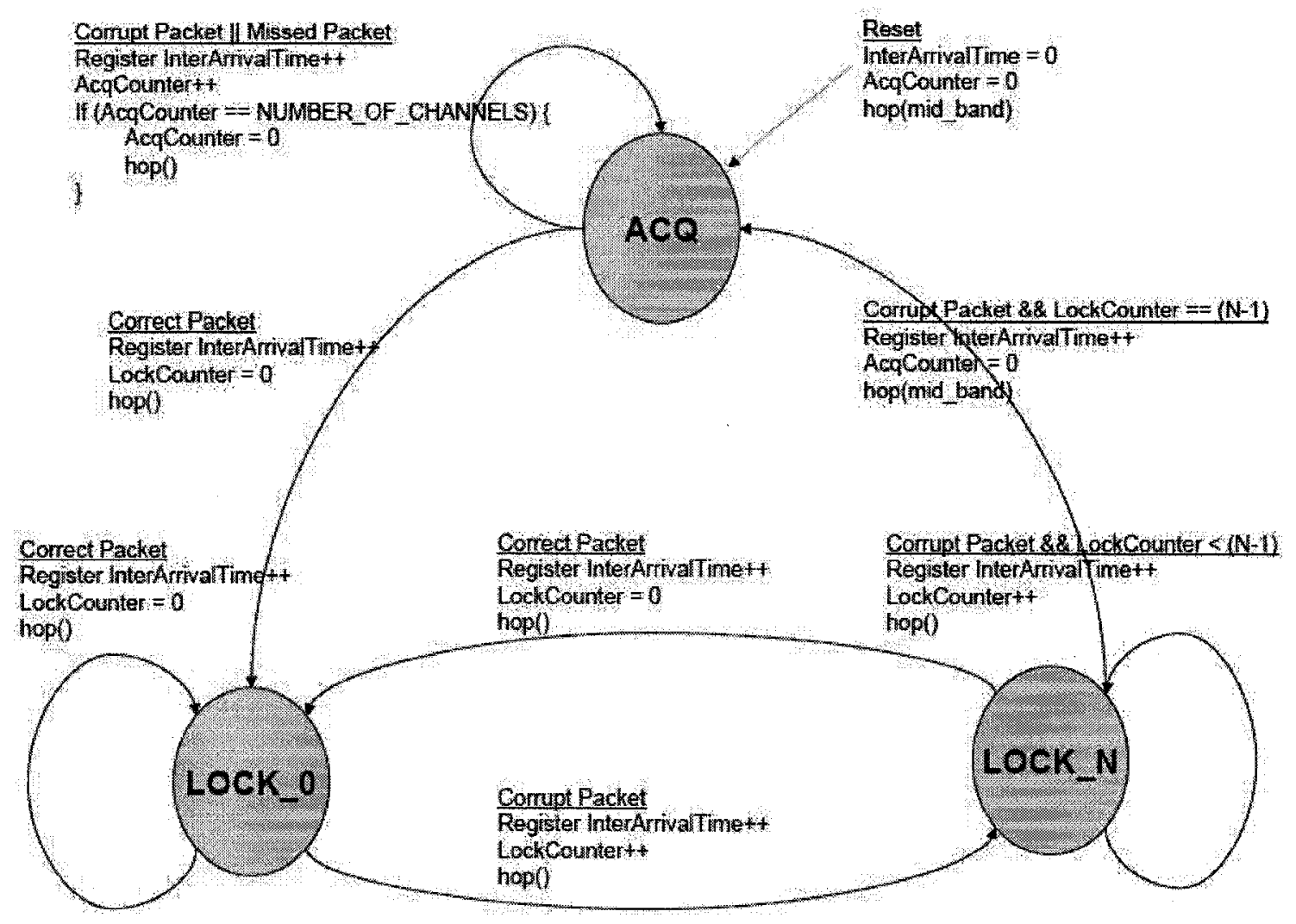

Figure 36: Receiver FSM

The receiver continuously monitors the inter-arrival time of correctly received packets. If the inter-arrival time exceeds $H R T$ then a lag is registered. The reception of a correct packet represents a new opportunity for a lag to occur and is therefore also registered. SLOP is obtained at the end of a single run of an experiment by dividing the registered number of lags by the registered number of opportunities.

\subsection{Experimental Procedure}

One million packets are transmitted in each run of the experiment. Two experiments are carried out: one to plot $S L O P$ against $P_{D}$, and the other to plot $S L O P$ against $N$. The purpose of these experiments is to verify the theoretical results. 
I refrained from plotting $S L O P$ against $P_{D}$, although it would have been the ideal scenario. Finding $P_{D}$ for each run of the experiment requires running a $P E R$ test in each of the channels then finding the average PER. To get the same resolution as SLOP, one million packets would have to be transmitted per channel. This roughly corresponds to 16 minutes per channel. There are 40 channels in the band, which means that an experiment to measure a single point on the $S L O P$ vs. $P_{D}$ plot takes roughly 11 hours, an impractical solution.

Instead, I decided to plot SLOP against the received power at the antenna port (Effective Isotropic Received Power, EIRP). In an environment perturbed only by AWGN, the received power would be directly proportional to the $S N R$ at the receiver port. The received power can be easily measured using a spectrum analyzer, and can be easily varied using a variable attenuator.

The $2^{\text {nd }}$ experiment plots $S L O P$ against $N$. The plot is drawn at a fixed received power point selected from the plot of the previous experiment (SLOP vs. EIRP). Again, the reason being that received power is easier to measure than $\mathrm{P}_{\mathrm{D}}$, but is proportional to $P_{D}$.

\subsubsection{SLOP VS EIRP}

To plot $S L O P$ against the received power, a variable attenuator is placed in between the transmitter and the receiver as shown in the Figure $\mathbf{3 7}$ below. To obtain a single point $(S L O P, E I R P), N$ is fixed, attenuation is gradually increased and the experiment re-ran for 
a period of 16 minutes (1million packets). At the end of the experiment, the setting of the attenuator is noted, and the measured SLOP is noted.

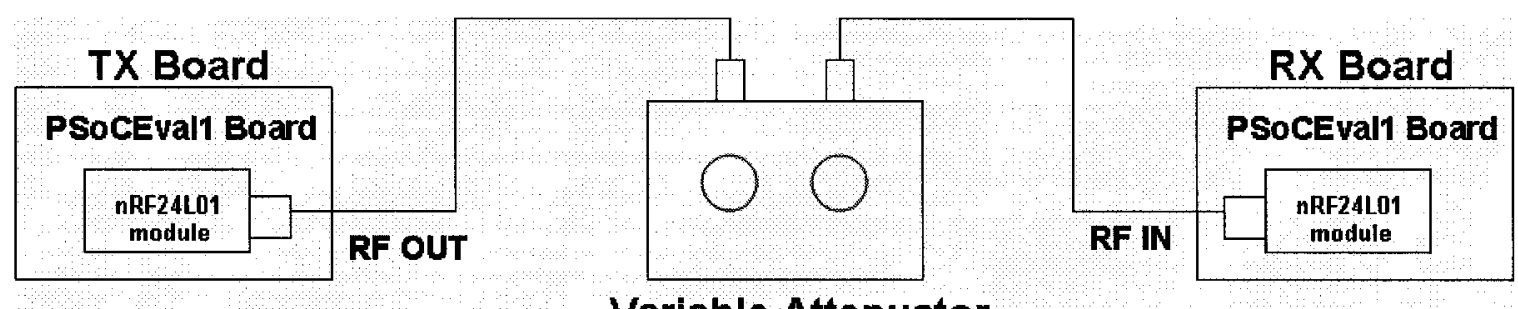

Figure 37: Attenuator test setup

Roughly 30 runs of the experiment were made at attenuation levels ranging from $0 \mathrm{~dB}$ to $70 \mathrm{~dB}$. The next step of the experiment is to measure the actual received power at the attenuator settings which were used in the experiment.

To measure the received power, the transmitter is set to transmit a continuous carrier. The output of the attenuator is connected to a spectrum analyzer. The spectrum analyzer is set at the center frequency of the carrier. The resolution bandwidth and the video bandwidth of the spectrum analyzer are both set to $2 \mathrm{MHz}$, and the span is set to $5 \mathrm{MHz}$.

Each of the attenuator settings used in the previous experiment is then set, and the received power measured by the spectrum analyzer is noted. Note that this provides for an accurate method of measuring the received power since it also accounts for any losses that occur through the coaxial cables and the matching networks. The setup for measuring the Received Power is shown in Figure 38 below. 


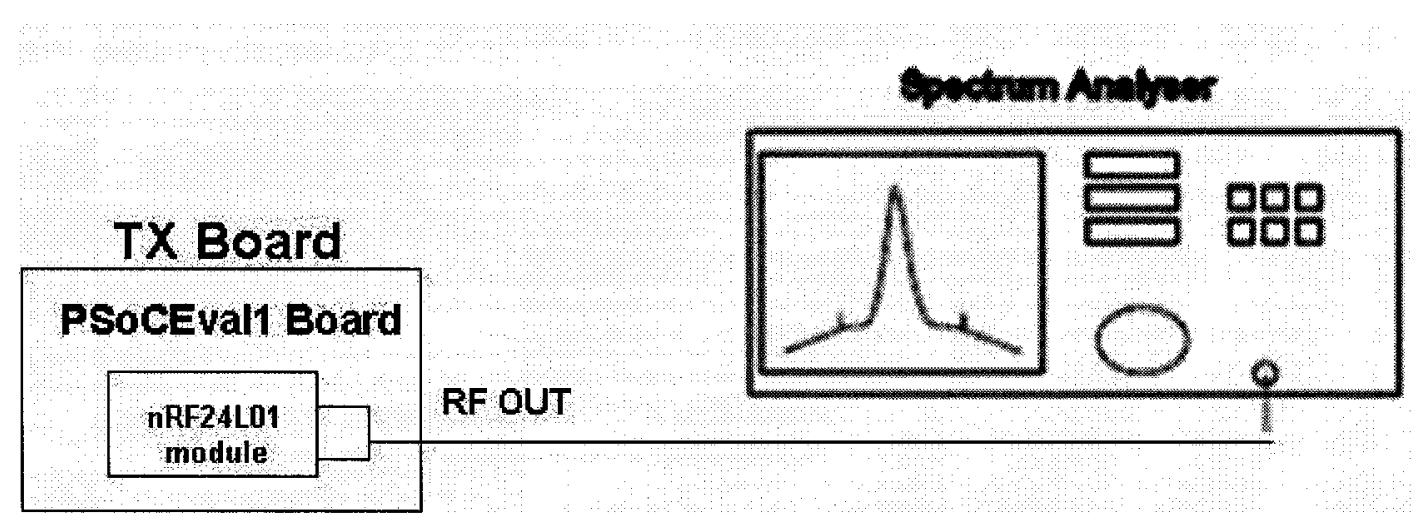

Figure 38: Test setup for measuring Received Power

\subsubsection{SLOP Vs $N$}

The experimental setup used to plot SLOP against $N$ is similar to the one used in the previous experiment. The procedure differs however. In this experiment, EIRP is fixed at an arbitrary point, and $N$ is varied instead. SLOP is measured and noted at every $N$. SLOP Vs $N$ is plotted at the end of the experiment.

\subsection{Results}

The first experiment plots $S L O P$ vs. EIRP. The resolution of the plot is low, due to the difficulty and time it would take to obtain a high-resolution plot. The result is shown in Figure 39 below. The figure shows that $S L O P$ improves as the received power increases (therefore as $P_{D}$ increases), in agreement with theory and simulation. 


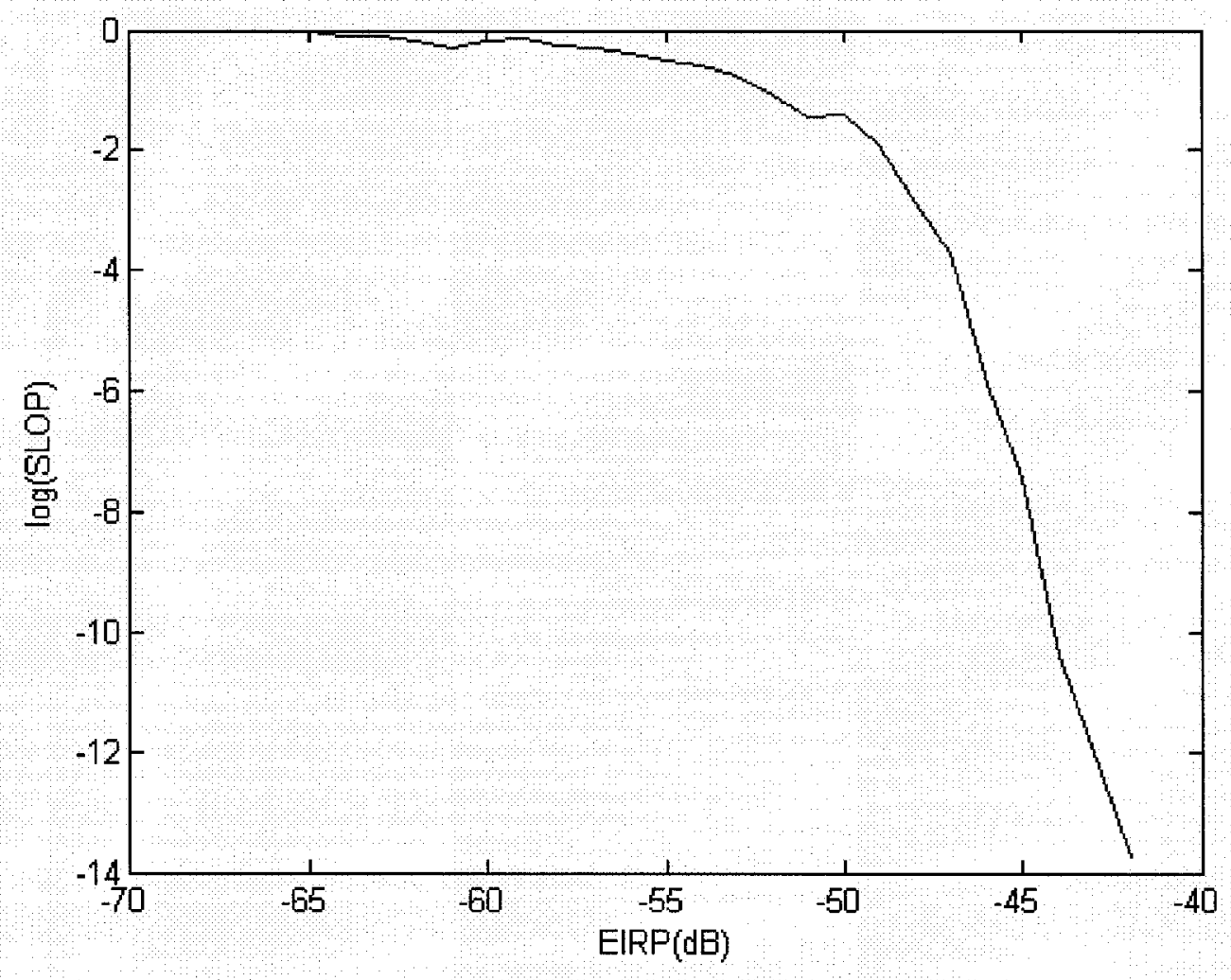

Figure 39: SLOP Vs Received Power. $(\mathrm{N}=3)$

The second experiment plots $S L O P$ vs. $N$. Again, the purpose of this experiment is to verify the related theoretical and simulation results. The plot is shown in Figure 40 below. Plots for the theoretical and simulation results are added to the figure for comparison purposes.

Figure 40 clearly shows the agreement of the experimental result with the theoretical and the simulation results. I believe this validates the theoretical derivation of SLOP made in the theory section, and also validates modeling acquisition time as an exponential random variable. 


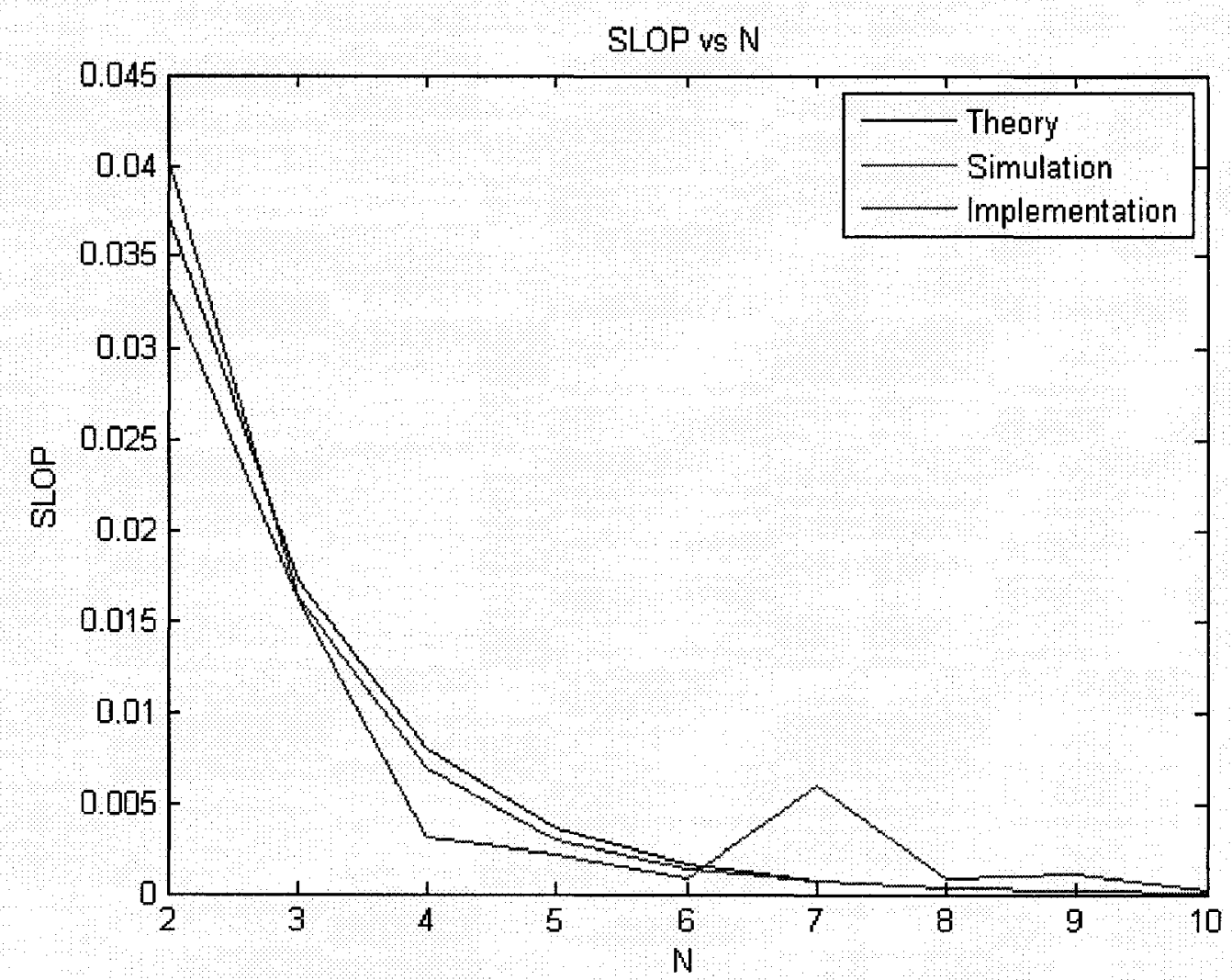

Figure 40: $S L O P$ Vs N. $\left(\right.$ Received Power $\left.=-75 \mathrm{dBm}, P_{D}=0.55\right)$ 


\section{Summarized Conclusion and Future Work}

ISM transceivers are basic building blocks in the practical design of radio control systems. The system-level architecture of currently available ISM transceivers can be classified into five generations, depending on the amount of silicon integration available in the transceiver.

In addition to the extensive integration features found in ISM transceivers, there exist a number of noise and interference-resistance techniques which are used in practice to establish a robust communication channel in the ISM band. Available noise and interference resistance techniques include FEC, DSSS, and FHSS. Of those techniques, frequency hopping is found to be the only technique available which enables the maximization of interference resistance in the ISM band. This is due to the hardware limitations placed by the current state of the art in ISM transceivers on applications that require maximizing interference-resistance. However in reality frequency hopping may not necessarily be the optimal solution. Therefore as an alternative to the use of ISM transceivers in the design of radio control systems, custom transceiver architecture can be proposed which optimizes performance of radio control systems in the ISM band. This is left for future work.

The performance of radio control systems depends on the interarrival time of correctlyreceived packets. A lag occurs when the interarrival time of correctly received packets 
exceeds the Human Response time (HRT). The System Lag Occurrence Probability $(S L O P)$ is the probability of a lag occurring during a radio control session. SLOP is used to characterize performance of real-time radio control systems in the ISM band. Imperfections in the wireless channel (i.e. noise and interference) increase SLOP and therefore degrade performance. Frequency hopping is used in practice to mitigate the effect of unpredictable interference present in the ISM band.

On the average, a frequency hopping system switches periodically between two modes: acquisition and tracking. Many acquisition and tracking engines are found in the literature. Uniform serial acquisition and $\mathrm{N}$-state lock detector tracking are chosen in this thesis because they simplify analysis and satisfy the conditions required for the derivation of SLOP. In practice, other acquisition and tracking engines such as those employed in Adaptive Frequency Hopping systems can be used to improve performance. SLOP can be derived based on the new proposed schemes in a similar manner to the derivation shown in Chapter 3. The use of more complex acquisition and tracking engines and the analysis of their effects on the performance of radio control systems is left for future work.

The derivation of SLOP places emphasis on the PER parameter rather than physical layer parameters such as BER or SINR. This is done to abstract any physical layer effects and keep the focus of the thesis on real-time radio control application design. Such physical layer parameters include the wireless channel model, the type and pattern of interference present in the band, and the type of wireless modulation used in communication. 
Studying the effect of these and other physical layer parameters on SLOP is left for future work.

To complete the derivation of $S L O P$, Chapter 3 models the acquisition time as an exponential random variable. Simulation and implementation results are presented in Chapters 4 and 5 to validate modeling the acquisition time as an exponential random variable. Specifically, theoretical and simulation curves are plotted and compared for SLOP vs. $P_{D}$ and SLOP vs. N. The simulation results are in perfect agreement with the theoretical curves, which are taken to validate modeling the acquisition time as an exponential random variable. However, further validation of this model can be made by running a simulation which measures a large number of acquisition times and plots their histogram. Such validation is left for future work.

This thesis is mainly concerned with the performance of radio control systems operating in the presence of noise and interference. Therefore, the simulator built to study the performance of a frequency hopping radio control system considers only the effect of noise and interference on the performance of the system. However, frequency hopping can also be used in Code-Division Multiple-Access (CDMA) operation. In CDMA systems, multiple users are assigned a set of orthogonal hopping sequences which enable the users to share the band with minimal self-interference. In such a system, selfinterference as well as external interference can degrade performance. The simulator presented in Chapter 4 and the experimental setup presented in Chapter 5 can be extended 
to test CDMA operation, and study the effect of self-interference on the performance of a frequency hopping radio control system. Such extensions are left for future work. 


\section{Bibliography}

[1] Alberto Leon Garcia, "Probability and Random Processes for Electrical Engineering", AddisonWesley Publishing Company, $2^{\text {nd }}$ Edition, May 1994

[2] Analog Devices, "High Performance ISM Band Transceiver IC - ADF7025", Analog Devices Datasheet Rev. A, 2006

[3] Andreas Polydoros and Charles Weber, "A Unified Approach to Serial Search Spread Spectrum Code Acquisition - Part I: General Theory", IEEE Transactions on Communications, Vol. COM32, No. 5, May 1984

[4] Andreas Wilde, "Extended Tracking Range Delay Lock Loop", German Aerospace Research Establishment, Germany, IEEE, 1995

[5] Aroosh R. Elahi, "Architecture of an Advanced Synthesized Receiver for Remote Control Applications", Carleton University, M.A.Sc. Thesis, June 122008

[6] Atmel, “ZigBee IEEE 802.15.4 Radio Transceiver: AT86RF230”, Atmel, May 22 2005

[7] Bernard Sklar, "Digital Communication: Fundamentals and Applications", Chapter $1,2^{\text {nd }}$ Edition, Prentice Hall, Upper Saddle River, New Jersey, 2001

[8] Chipcon, "CC1010: Single Chip Very Low Power RF Transceiver with 8051-Compatible Microcontroller", Texas Instruments Datasheet SWRS047, December 122004

[9] Chipcon, "CC1100: Single Chip Low Cost Low Power Transceiver", Datasheet SWRS038A, Texas Instruments, 2006

[10] Chipcon, "CC1110Fx/CC1111Fx, Low Power Sub 1GHz RF System on Chip (SoC) With MCU, Memory, Transceiver, and USB Controller", Texas Instruments Datasheet SWRS033B, September 142007

[11] Chipcon, "CC2500: Single Chip Low Cost Low Power Transceiver", Texas Instruments Datasheet SWRS040A, 2006

[12] Chipcon, "CC2510Fx/CC2511Fx, True System on Chip with Low Power RF Transceiver and MCU", Texas Instruments Datasheet SWRS055A, July 62006 
[13] Cody, W. J., "Rational Chebyshev Approximations for the Error Function", Math. Comp., pgs. $631-638,1969$

[14] Cypress Microsystems, "CYWUSB6953, WirelessUSB PRoC Flash Programmable MCU + Radio", Cypress Semiconductor Corporation Document \# 38-16017 Rev C, August 192005

[15] Cypress Microsystems, "PSoC Mixed Signal Array CY8C29466", Cypress Microsystems Inc Document No. 38-12013, November 122004

[16] Cypress Semiconductor, "CYRF6936: WirelessUSB LP 2.4 GHz Radio SoC", Cypress Semiconductor, April $2^{\text {nd }} 2007$

[17] Cypress Semiconductor, "CYWUSB6935: WirelessUSB LR 2.4 GHz DSSS Radio SoC”, Cypress Semiconductor, Datasheet, November 182004

[18] Cypress Semiconductor, "PSoC Designer: IDE User Manual", Cypress Semiconductor, Document \# 38-12001, Rev. E, 2005

[19] Cypress Semiconductor, "PSoC Mixed Signal Array: Technical Reference Manual", Cypress Semiconductor, PSoC TRM version 2.10, 2006

[20] David M. Dicarlo and Charles L. Webber, "Multiple Dwell Serial Search: Performance and Application to Direct Sequence Code Acquisition", IEEE Transactions on Communications, Vol. COM-31, No. 5, May 1983

[21] Duncan Dlugos, Robert Scholtz, "Acquisition of Spread Spectrum Signals by an Adaptive Array", IEEE Transaction on Acoustics, Speech, and Signal Processing, Vol. 1 37, No. 8, August 1989

[22] F Dominique, J. H. Reed, "Robust Frequency Hop Synchronization Algorithm", Electronics Letters Online, Volume 32, No. 16, August 1996

[23] Fairchild Semiconductor, "RMPA2455: 2.4-2.5 GHz 1 Watt InGaP HBT Linear Power Amplifier", Fairchild Semiconductor, Datasheet RMPA2455 Rev. F, Sept. 2005

[24] Federal Communications Commission, "Part 15 - Radio Frequency Devices", FCC, 47 CFR Part 15, September 192005

[25] G.H Mowbry, J. W. Gebbard, “Man's Senses as Informational Channels”, In Human Factors in the Design and Use of Control System, edited by H.W. Sinaiko, 115-149, New York: Dover, 1961

[26] Integration, “IA4432 ISM Transceiver”, Silicon Labs Wireless Datasheet IA4432-DS, 2007 
[27] Jack K Holmes and Chang C Chen, "Acquisition Time Performance of PN Spread-Spectrum Systems", IEEE Transactions on Communications, Vol. COM-25, No. 8, August 1977

[28] James Han, Donald Lanzinger, Dean Sklar, “Assessing the Performance of Packet Retransmission Schemes Over Satellite Link", IEEE, 2006

[29] James M. Abatti, "Small Power: The Role of Micro and Small UAVs in the Future", Centre for Strategy and Technology, Air War College, Air University, November 2005

[30] John Rogers, Calvin Plett, "Radio Frequency Integrated Circuit Design”, Chapter 2, Artech House, Norwood MA, 2003

[31] Jonathan Min, Henry Samueli, "Synchronization Techniques for a Frequency-Hopped Wireless Transceiver", IEEE $46^{\text {th }}$ Issue, May 1996, Pages: $183-187$ vol. 1

[32] K.H. Torvmark, "Frequency Hopping Systems", Application Note SWRA077, Texas Instruments

[33] Kapil Chawla, Dilip Sarwate, "Parallel Acquisition of PN Sequences in DS/SS Systems", IEEE Transactions on Communications, Vol. 42, No. 5, May 1994

[34] Mingru Zhu, Keith Chugg, "Iterative Message passing Techniques for Rapid Code Acquisition", IEEE, 2003

[35] Nordic Semiconductor, "External PA for nRF24xx", Nrodic Semiconductor Reference Design nRD24-01, May $24^{\text {th }} 2005$

[36] Nordic Semiconductor, "nRF2401 Single Chip 2.4 GHz Transceiver", Nordic Semiconductor, Datasheet, March 2006

[37] Nordic Semiconductor, "nRF24E1: 2.4GHz RF Transceiver with Embedded 8051 Compatible Microcontroller and 9 Input, 10 bit ADC", Nordic Semiconductor, Datasheet, March 2007

[38] Nordic Semiconductor, "nRF24L01 Single Chip 2.4 GHz Transceiver”, September 2006

[39] Nordic Semiconductor, "nRF24LU1, Single Chip 2.4GHz Transceiver with USB Microcontroller and Flash Memory", Nordic Semiconductor Objective Product Specification v1.1, August 2007

[40] O. Grotmol, "Frequency Hopping Protocol for CC1010", Application Note SWRA068, Texas Instruments, 2003

[41] Peter Spevak, "Implementing a Bidirectional, Half-Duplex FSK RF Link With TRF6900 and MSP430", Texas Instruments, Application Note SLAA121, March 2001 
[42] Philips, "BGA6589: MMIC Wideband Medium Power Amplifier", Philips, September $19^{\text {th }} 2003$

[43] Robert C. Dixon, "Spread Spectrum System: With Commercial Applications", Third Edition, John Wiley and Sons, New York, 1994

[44] Robin Hoel, "FEC Implementation", Application Report SWRA113, Texas Instruments, 2006

[45] Roger L Peterson, Rodger E Ziemer, David E Borth, "Introduction to Spread Spectrum Communications", Prentice Hall, New Jersey, 1995

[46] Savo Glisic, Branka Vucetic, "Spread Spectrum CDMA Systems for Wireless Communications", Artech HouseInc: Boston, 1997

[47] Semtech Corporation, "FCC Regulations for ISM Band Devices: 9-2 - $928 \mathrm{MHz}$ ", Semtech, Application Note AN1200.04, 2006

[48] Semtech Corporation, "SX1211 Transceiver, Lower Power Integrated UHF Transceiver, Short Range Devices", Advanced Communications and Sensing Products Division, August 152007

[49] Si-ming Pan and David Dodds, "Acquisition Time Distribution for Spread Spectrum Receivers", IEEE Journal on Selected Areas in Communications, Vol. 8, No. 5, June 1990

[50] Sun Microsystems, "Sun Developer Network (SDN): The Source for Java Developers", Sun Microsystems Inc, 2008, Available at: http://java.sun.com

[51] Texas Instruments, TRF6900A Single-Chip RF Transceiver, Literature Number SLAS258D, Sep 2000

[52] Texas Instruments, Understanding and Enhancing Sensitivity in Receivers for Wireless Applications, Literature Number SWRA030, May 1999

[53] Carlo Kopp, "An Introduction to Spread Spectrum Techniques", May 1997, Available at: http://www.csse.monash.edu.au/ carlo/SYSTEMS/Spread-Spectrum-0597.htm

[54] DATA-LINC Group, "Spread Spectrum Diagram: Typical Frequency Hopping Pattern", Articles and Technical 2006, Avapers, at: http://www.wwsinternational.com.au/DataLinc/articles/spsptech.htm

[55] Hoi-Sheung So, Giang Nguyen, Jean Walrand, "Practical Synchronization Techniques for MultiChannel MAC", Department of Electrical Engineering and Computer Sciences, University of California, CA, 2006 
[56] Cypress Semiconductor, "WirelessUSB PRoC DVK Quick Start Guide", Available at www.cypress.com

[57] Mark Hastings, "CY3210 PSoC Evall Schematic", Cypress Semiconductor, Available at www.cypress.com

[58] Sparkfun Electronics, "nRF24L01 Breakout Board", Available at www.sparkfun.com

[59] Theodore Vlachos and Evaggelos Geraniotis, "Performance Study of Hybrid Spectrum System Random-Access Communications", IEEE Transactions on Communications, Vol. 39, No. 6, June 1991

[60] L. Sadiq and A. Hamid Aghvami, "Performance of an Asynchronous Hybrid Spread Spectrum System in the Presence of Interference, Rician Fading, and AWGN", IEE Proceedings-I, Vol. 138, No. 2, April 1991

[61] R. Muammar, "Performance Evaluation of a Hybrid Spread Spectrum System in a Hostile Land Mobile Radio Channel", University of Illinois at Chicago, EECS Department, 1991

[62] Bruce F. McGuffin, "Jammed FH-FSK Performance in Rayleigh and Nakagami-M Fading", Lincoln Laboratory, MIT, Massachusetts, 2003

[63] Kah C. Teh, Alex C. Kot, and Kwok H. Li, "Partial Band Jammer Suppression in FFH SpreadSpectrum System Using FFT", IEEE Transactions on Vehicular Technology, Vol. 48, No. 2, March 1999

[64] Amer A. Hassan, Wayne E. Start, and John E. Hershey, "Frequency-Hopped Spread Spectrum in the Presence of a Follower Partial-Band Jammer", IEEE Transactions on Communications, Vol. 41, No. 7, July 1993

[65] Leonard E. Miller, Johng S. Lee, Robert H. French, and Don J. Torrieri, "Analysis of an Antijam FH Acquisition Scheme", IEEE Transaction on Communications, Vol. 40, No. 1, January 1992

[66] J. Zander and G. Malmgren, "Adaptive Frequency Hopping in HF Communications", IEE Proc.Commun., Vol. 142, No. 2, April 1995

[67] Zhang Shaoyuan, Yao Fuquang, "Research on the Adaptive Frequency Hopping Technique in the Correlated Hopping Enhanced Spread Spectrum Communication", $4^{\text {th }}$ International Conference on Microwave and Millimeter Wave Technology Proceedings, 2004 
[68] David M. Dicarlo and Charles L. Weber, "Statistical Performance of Single Dwell Serial Synchronization Systems", IEEE Transactions on Communications, Vol. Com-28, No. 8, August 1980

[69] Vladan M. Jovanovic, "On the Distribution Function of the Spread-Spectrum Code Acquisition Time", IEEE Journal on Selected Areas in Communications, Vol. 10, No. 4, May 1992 